\title{
FOUNDATIONS OF A GENERAL THEORY OF BIRATIONAL CORRESPONDENCES
}

\author{
BY \\ OSCAR ZARISKI
}

In our papers dealing with the reduction of singularities of an algebraic surface (see $[8,11]$ ), we were forced to devote a good deal of space to certain properties of birational correspondences for which we could find no general proofs in the literature. These properties were of a general character and therefore could not be regarded as part of the reduction proof proper, although they did play an auxiliary role in the proof. A similar situation arose in our reduction proof for three-dimensional varieties (not yet published), but in this case the amount of preliminary general material on birational correspondences used in the proof was even larger and was out of proportion to the length of the reduction proof proper. It thus became increasingly clear that the procedure of treating general questions of birational correspondences only as and when these questions come up in connection with various steps of the reduction process, could no longer be followed in the case of higher varieties. Instead it seemed necessary-and also worth while for its own saketo develop systematically in a separate paper the fundamental concepts and theorems of the theory of birational correspondences, and to do this in as general a fashion as possible. This we propose to do in the present paper. We deal here with algebraic varieties, with or without singularities, over an arbitrary ground field (of characteristic zero or $p$ ).

It is difficult to say which of our results are entirely novel and which are not. Since many of the results hold only for normal varieties, they would appear to be novel inasmuch as our concept of a normal variety is new. On the other hand, most of our results were known for nonsingular models. It is perhaps correct to say that the novelty of the present investigation consists in showing that most of the known properties of birational correspondences between nonsingular varieties remain true more generally for normal varieties.

Of importance for the theory of algebraic functions over arbitrary ground fields of characteristic $p$ is the fact that our construction of normal varieties which we gave in [7] in the case of algebraically closed ground fields of characteristic zero-and which carries over without essential modifications to arbitrary fields of characteristic zero (see II. 2) - can be extended to arbitrary fields of characteristic $p$ (II. 2). This extension is made possible by a theorem of F. K. Schmidt [5] and by the normalization theorem of Emmy

Presented in part to the Society, December 31, 1941 under the title Normal varieties and birational correspondences (see [12]); received by the editors September 1, 1942. 
Noether. The proof of this last theorem for arbitrary ground fields (and not only for fields containing "sufficiently many" elements; see Krull [2, pp. 41$42]$ ) is given in II.2.

A feature of the treatment is our use of valuation theory. Our very definition of a birational correspondence (II.1) is valuation-theoretic in character, and our proofs are naturally conditioned by this valuation-theoretic approach. The characterization of an integrally closed ring as intersection of valuation rings, and the ideal theory in such a ring, also play an important role in our treatment.

Thanks are due to Irvin Cohen for valuable assistance lent by him during the preparation of this paper.

The following list of contents will give an idea of the individual topics treated.

\section{Contents}

Part I. Valuation-Theoretic PRELIMINARIES

1. Homogeneous ideals. . . . . . . . . . . . . . . . . . . . . . . . . . 491

2. Homogeneous and nonhomogeneous coordinates................. 494

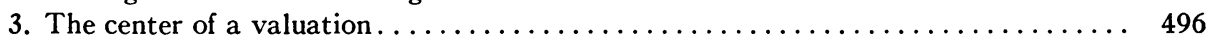

4. Existence theorems for valuations with a preassigned center . . . . . . . . . . 499

PART II. GENERAL THEORY OF BIRATIONAL CORRESPONDENCES

1. Valuation-theoretic definition of a birational correspondence . . . . . . . . 505

2. The birational correspondence between $V$ and a derived normal model $\bar{V} \ldots \ldots \ldots 506$

3. The fundamental elements of a birational correspondence . . . . . . . . . . 512

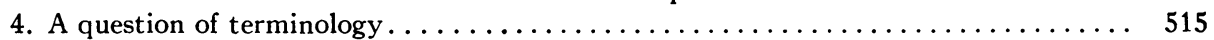

5. The join of two birationally equivalent varieties. $\ldots \ldots \ldots \ldots \ldots \ldots \ldots \ldots \ldots \ldots$

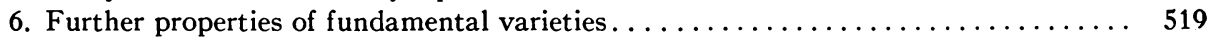

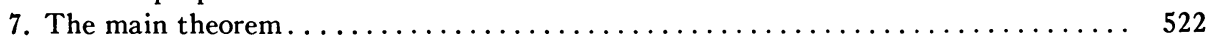

8. Continuation of the proof of the main theorem $\ldots \ldots \ldots \ldots \ldots \ldots \ldots \ldots \ldots \ldots$

9. The fundamental locus of a birational correspondence $\ldots \ldots \ldots \ldots \ldots \ldots \ldots \ldots \ldots 27$

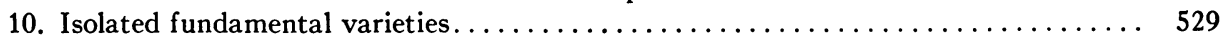

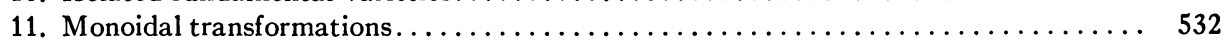

\section{Part I. Valuation-Theoretic PRELIMINARIES}

1. Homogeneous ideals. Let $\eta_{0}, \eta_{1}, \cdots, \eta_{n}$ be the homogeneous coordinates of the general point of an irreducible $r$-dimensional algebraic variety $V$ immersed in an $n$-dimensional projective space. The coordinates $\eta_{i}$ are defined to within a linear homogeneous nonsingular transformation with coefficients in the ground field K. By the very definition of homogeneous coordinates (Zariski $[7$, p. 284]), if $f(\eta)=0$ is an algebraic relation between the $\eta$ 's over $\mathrm{K}$, and if we write $f$ as a sum of forms $f_{i}(\eta)$ of different degrees, then each $f_{i}(\eta)$ individually is zero. This is equivalent to saying that the polynomials $f(y)$ in the polynomial ring $\mathrm{K}\left[y_{0}, y_{1}, \cdots, y_{n}\right]$ (the $y^{\prime}$ s are indeterminates) such that $f(\eta)=0$, form a homogeneous ideal, that is, an ideal which has a base consisting of forms. 
Let $P$ denote the ring $\mathrm{K}\left[\eta_{0}, \eta_{1}, \cdots, \eta_{n}\right]$. We shall also consider homogeneous ideals in $P$, that is, again ideals in $P$ which have a base consisting of forms. These are the ideals on which the homogeneous ideals of $\mathrm{K}\left[y_{0}, \cdots, y_{n}\right]$ are mapped in the homomorphism $\mathrm{K}[y] \sim \mathrm{K}[\eta]$. The ring $P$ possesses relative automorphisms $\tau_{\lambda}$ over $\mathrm{K}$, where for any element $\phi(\eta)$ in $P$ we define: $\tau_{\lambda}(\phi(\eta))=\phi(\lambda \eta), \lambda \in \mathrm{K}, \lambda \neq 0$. It is clear that if $\mathfrak{A}$ is a homogeneous ideal in $P$, then $\tau_{\lambda}(\mathfrak{U})=\mathfrak{A}$. Conversely, we have the following theorem:

THEOREM 1. If $\mathrm{K}$ has infinitely many elements and if an ideal $\mathfrak{A}$ in $P$ is such that $\tau_{\lambda}(\mathfrak{U}) \subseteq \mathfrak{A}$, for all $\lambda$ in $\mathrm{K}(\lambda \neq 0)$, then $\mathfrak{A}$ is homogeneous.

Proof. Let $\omega=f(\eta)$ be an arbitrary element of $\mathfrak{A}$ and let $f(\eta)=f_{s}(\eta)+f_{s+1}(\eta)$ $+\cdots+f_{m}(\eta)$, where $f_{i}$ is a form of degree $i$. We take arbitrarily in $\mathrm{K}$ a set of $m-s+1$ distinct elements $\lambda_{1}, \lambda_{2}, \cdots, \lambda_{m-8+1}$, all different from zero. We have, by hypothesis:

$$
\tau_{\lambda_{i}}(\omega)=\lambda_{i}^{*} f_{s}(\eta)+\lambda_{i}^{*+1} f_{s+1}(\eta)+\cdots+\lambda_{i}^{m} f_{m}(\eta) \equiv 0(\mathfrak{A}) .
$$

These $m-s+1$ congruences imply the congruences: $f_{j}(\eta)=0, j=s$, $s+1, \cdots, m$. Hence $\mathfrak{A}$ is a homogeneous ideal, as was asserted.

Theorem 2. If $\mathfrak{A}=\left[\mathfrak{q}_{1}, \mathfrak{q}_{2}, \cdots, \mathfrak{q}_{h}\right]$ is a normal decomposition of a homogeneous ideal $\mathfrak{A}$ into maximal primary components, then each $\mathfrak{q}_{i}$ is either itself homogeneous or-in the case of an embedded component-can be so selected as to be homogeneous.

Proof. We first consider the case of an infinite ground field $\mathrm{K}$. Let $\mathfrak{p}_{i}$ be the prime ideal associated with the primary ideal $\mathfrak{q}_{i}$. The infinitely many automorphisms $\tau_{\lambda}$ leave $\mathfrak{A}$ invariant and hence must permute the prime ideals $\mathfrak{p}_{1}, \mathfrak{p}_{2}, \cdots, \mathfrak{p}_{h}$. Consequently each of these prime ideals is left invariant by infinitely many automorphisms $\tau_{\lambda}$. It follows then from the proof of the preceding theorem, that the prime ideals $\mathfrak{p}_{i}$ of $\mathfrak{A}$ must be all homogeneous.

If $\mathfrak{p}_{i}$ is an isolated prime ideal of $\mathfrak{A}$, then $\mathfrak{q}_{i}$ is uniquely determined. Since $\tau_{\lambda}\left(\mathfrak{p}_{i}\right)=\mathfrak{p}_{i}$, it follows that also $\tau_{\lambda}\left(\mathfrak{q}_{i}\right)=\mathfrak{q}_{i}$, whence $\mathfrak{q}_{i}$ is homogeneous.

Let now $\mathfrak{p}_{i}$ be an embedded prime of $\mathfrak{A}$. We apply to $\mathfrak{q}_{i}$ all the automorphisms $\tau_{\lambda}(\lambda \in K, \lambda \neq 0)$ and we denote by $q_{i}^{*}$ the intersection of all the ideals $\tau_{\lambda}\left(\mathfrak{q}_{i}\right)$. From the very definition of a primary ideal, it follows immediately that $\mathfrak{q}_{i}^{*}$ is a primary ideal and that $\mathfrak{p}_{i}$ is its associated prime ideal. Moreover, by Theorem $1, \mathfrak{q}_{i}^{*}$ is homogeneous, and since $\mathfrak{A} \subseteq \mathfrak{q}_{i}^{*} \subseteq \mathfrak{q}_{i}$, we find: $\mathfrak{A}=\left[\mathfrak{q}_{1}, \mathfrak{q}_{2}, \cdots, \mathfrak{q}_{i}^{*}, \cdots, \mathfrak{q}_{h}\right]$, as was asserted.

Let now $\mathrm{K}$ be a finite field. We adjoin to $\mathrm{K}$ a transcendental $u$ and we put $\mathrm{K}^{*}=\mathrm{K}(u), P^{*}=\mathrm{K}^{*}\left[\eta_{0}, \eta_{1}, \cdots, \eta_{n}\right]$ (we assume that $u$ is also a transcendental with respect to the ring $P$ ). By the preceding case, our theorem holds for the ring $P^{*}$ over the new ground field $\mathrm{K}^{*}$. We can draw from this the conclusion that the theorem also holds for the ring $P$, provided we first es- 
tablish certain relations between the ideals in $P$ and the ideals in $P^{*}$. If $\mathscr{B}$ is an ideal in $P$, it determines in $P^{*}$ the extended ideal $P^{*} \cdot \mathfrak{B}$. Vice versa, every ideal $\mathfrak{S}^{*}$ in $P^{*}$ gives rise to a contracted ideal in $P$, namely the ideal $\mathfrak{S}^{*} \cap P$ (but $\mathfrak{C}^{*}$ is not necessarily the extended ideal of its contracted ideal). Note that every element $\omega^{*}$ of $P^{*}$ can be written in the form:

$$
\omega^{*}=\frac{1}{g(u)} \cdot\left(\omega_{0} u^{m}+\omega_{1} u^{m-1}+\cdots+\omega_{m}\right),
$$

where $g(u) \in \mathrm{K}[u]$ and $\omega_{j} \in P$. The relations which we wish to establish concern the operations of extension and contraction just described, and are as follows:

a. An ideal $\mathfrak{B}^{*}$ in $P^{*}$ is the extension of an ideal in $P$ if and only if the congruence $\omega^{*} \equiv 0\left(\mathfrak{B}^{*}\right)$ implies $\omega_{j} \equiv 0\left(\mathfrak{B}^{*}\right)$, for $j=0,1, \cdots, m$, where $\omega^{*}$ is written in the form (1).

b. $P^{*} \cdot \mathfrak{B} \cap P=\mathfrak{B}$.

c. If $\mathfrak{B}_{1} \cap \mathfrak{B}_{2}=\mathfrak{B}_{3}$, then $P^{*} \cdot \mathfrak{B}_{1} \cap P^{*} \cdot \mathfrak{B}_{2}=P^{*} \cdot \mathfrak{B}_{3}$. The proofs are trivial.

d. If $\mathfrak{p}$ is a prime ideal in $P$, then $P^{*} \cdot \mathfrak{p}$ is also a prime ideal. This assertion is essentially equivalent to the well known theorem that if $R$ is an integral domain and if $u$ is a transcendental with respect to $R$, then $R[u]$ is also an integral domain. In the present case $R$ is the ring $P / \mathfrak{p}$.

Let now $\mathfrak{A}$ be a homogeneous ideal in $P$ and let us consider its extended ideal $P^{* \mathfrak{A}}$ in $P^{*}$. It is clear that also $P^{* \mathfrak{A}}$ is a homogeneous ideal in $P^{*}$. Since $\mathrm{K}^{*}$ is an infinite field, we can write

$$
P^{*} \cdot \mathfrak{A}=\left[\mathfrak{q}_{1}^{*}, \mathfrak{q}_{2}^{*}, \cdots, \mathfrak{q}_{h}^{*}\right],
$$

where each $\mathfrak{q}_{i}^{*}$ is a homogeneous primary ideal. If we put $\mathfrak{q}_{i}=\mathfrak{q}_{i}^{*} \cap P$, we find, by property $b$ :

$$
\mathfrak{A}=\left[\mathfrak{q}_{1}, \mathfrak{q}_{2}, \cdots, \mathfrak{q}_{h}\right] .
$$

It is obvious that if $\mathfrak{B}^{*}$ is a homogeneous ideal in $P^{*}$, then $\mathfrak{B}^{*} \cap P$ is also a homogeneous ideal. Hence the ideals ' $_{i}$ are all homogeneous. Since they are obviously primary ideals, our theorem follows in view of the unicity theorems concerning the decomposition of ideals into maximal primary components.

In addition to the relations $\mathrm{a}, \mathrm{b}, \mathrm{c}, \mathrm{d}$, we shall also have occasion to use the following property:

e. If $\mathfrak{q}$ is a primary ideal in $P$ and if $\mathfrak{p}$ is its associated prime ideal, then $P^{*} \cdot q$ is also primary and $P^{*} \cdot \mathfrak{p}$ is its associated prime ideal.

To prove e, we denote by $\mathfrak{p}^{*}$ the prime ideal $P^{*} \cdot \mathfrak{p}$ and we consider any prime ideal $\mathfrak{p}_{1}^{*}$ of $P^{*} \cdot \mathfrak{q}$. We have: $\mathfrak{p}_{1}^{*} \supseteq P^{*} \mathfrak{q} \supseteq \mathfrak{q}$, whence $\mathfrak{p}_{1}^{*} \cap P \supseteq \mathfrak{q}$. But since the contraction of a prime ideal is also a prime ideal, it follows that $p_{1}^{*} \cap P \supseteq p$, whence

$$
p_{1}^{*} \supseteq p^{*}
$$


Now let $\omega^{*}$ be an arbitrary element of $\mathfrak{p}_{1}^{*}$ and let us write $\omega^{*}$ in the form (1). Since $\mathfrak{p}_{1}^{*}$ is a prime ideal of $P^{*} \cdot \mathfrak{q}$, there exists in $P^{*}$ an element $\xi^{*}$ such that $\omega^{*} \cdot \xi^{*} \equiv 0\left(P^{*} \cdot \mathfrak{q}\right), \xi^{*} \not \equiv 0\left(P^{*} \cdot \mathfrak{q}\right)$. Let us also write $\xi^{*}$ in a form similar to (1):

$$
\xi^{*}=\frac{1}{h(u)} \cdot\left(\xi_{0} u^{\mu}+\xi_{1} u^{\mu-1}+\cdots+\xi_{\mu}\right), \quad h(u) \in \mathrm{K}[u], \xi_{j} \in P .
$$

Since $\xi^{*} \not \equiv 0\left(P^{*} \cdot \mathfrak{q}\right)$, not all the elements $\xi_{0}, \xi_{1}, \cdots, \xi_{\mu}$ are in $\mathfrak{q}$. We may assume that $\xi_{0} \not \equiv 0(q)$, since otherwise we can drop the term $\xi_{0} u^{\mu}$ (it is permissible to replace $\xi^{*}$ by any element of $P^{*}$ which is congruent to it modulo $\left.P^{*} \cdot q\right)$. Since $\omega^{*} \xi^{*} \equiv 0\left(P^{*} \mathfrak{q}\right)$, we must have, by a and $\mathrm{b}: \omega_{0} \xi_{0} \equiv 0(\mathfrak{q})$, and consequently $\omega_{0} \equiv 0(\mathfrak{p})$, since $\xi_{0} \not \equiv 0(q)$. From this we conclude, in view of (3), that the element

$$
\frac{1}{g(u)} \cdot\left\lfloor\omega_{1} u^{m-1}+\omega_{2} u^{m-2}+\cdots+\omega_{m}\right\rfloor
$$

also belongs to $p_{1}{ }^{*}$. Continuing in the same fashion with this new element of $\mathfrak{p}_{1}^{*}$, we conclude that $\omega_{0}, \omega_{1}, \cdots, \omega_{m}$ are all in $\mathfrak{p}$. Hence $\omega^{*} \in P^{*} \cdot \mathfrak{p}$. Since $\omega^{*}$ is an arbitrary element of $p_{1}^{*}$, it follows that $p_{1}^{*} \subseteq p^{*}$, and this, in view of (3), yields the relation: $\mathfrak{p}_{1}^{*}=p^{*}$. What we have shown is that $P^{*} \mathfrak{q}$ has only one prime ideal, namely $\mathfrak{p}^{*}$, and that is exactly what is asserted in e.

The relation $\mathrm{b}$ shows that there is $\mathrm{a}(1,1)$ correspondence between the ideals $\mathfrak{B}$ in $P$ and their extended ideals $P^{*} \mathfrak{B}$ in $P^{*}$, for $P^{*} \mathfrak{B}_{1}=P^{*} \mathfrak{B}_{2}$ implies $\mathfrak{B}_{1}=\mathfrak{B}_{2}$. By the property $\mathrm{c}$, this correspondence is an isomorphism with respect to the operation $\cap$ of intersection. It is a straightforward matter to show that this correspondence is also an isomorphism with respect to the other elementary operations on ideals, namely the operation of forming the sum, the product and the quotient of two ideals:

$\mathrm{c}_{1} . P^{*} \cdot(\mathfrak{A}, \mathfrak{B})=\left(P^{*} \mathfrak{A}, P^{*} \mathfrak{B}\right)$.

c. $P^{*} \cdot(\mathfrak{A} \mathfrak{B})=P^{* \mathfrak{A}} \cdot P^{*} \mathfrak{B}$.

c.. $P^{*} \cdot(\mathfrak{A}: \mathfrak{B})=P^{*} \mathfrak{A}: P^{*} \mathfrak{B}$.

We shall refer to the relations $\mathrm{c}, \mathrm{c}_{1}, \mathrm{c}_{2}$ and $\mathrm{c}_{3}$ as the isomorphism relations. The question whether any one of these relations holds, arises quite generally whenever we deal with ideals in two rings $P, P^{*}$ such that $P$ is a subring of $P^{*}$. For a general treatment of the relationship between the ideals in two such rings, see Grell [1].

The ideal $\left(\eta_{0}, \eta_{1}, \cdots, \eta_{n}\right)$ is referred to as the irrelevant prime ideal in $P$. Any primary ideal whose associated prime ideal is the irrelevant prime is also referred to as irrelevant. Any prime homogeneous ideal, other than the irrelevant prime, is of dimension $s+1, s \geqq 0$, and defines an irreducible $s$-dimensional subvariety of $V$. Two homogeneous ideals which differ only by the irrelevant component define one and the same subvariety of $V$.

2. Homogeneous and nonhomogeneous coordinates. A preference for one or the other type of coordinates is in part a matter of taste. However, it can 
be claimed that in the study of properties of algebraic varieties, the use of homogeneous coordinates is indicated whenever one deals with properties in the large. For instance, the concept of a normal variety (Zariski $[7$, p. 285]) is defined essentially in terms of homogeneous coordinates. On the contrary, in questions pertaining to local properties it is preferable to use nonhomogeneous coordinates. Thus, if our attention is focused on a given subvariety $W$ of $V$ and if, say, $\eta_{0} \neq 0$ on $W$, that is, if $\eta_{0}$ does not belong to the homogeneous prime ideal by which $W$ is defined (whence $W$ does not lie in the hyperplane $y_{0}=0$ ), then we may find it convenient to pass to the nonhomogeneous coordinates $\xi_{i}=\eta_{i} / \eta_{0}, i=1,2, \cdots, n$. With respect to these coordinates " $W$ is at finite distance" - an expression that we shall use consistently. More generally, if $l=c_{0} \eta_{0}+c_{1} \eta_{1}+\cdots+c_{n} \eta_{n} \neq 0$ on $W, c_{i} \in \mathrm{K}$, and if, say $c_{\alpha} \neq 0$, then the quotients $\eta_{i} / l, i \neq \alpha$, may be equally well used as nonhomogeneous coordinates $\xi_{i}$ of the general point of $V$.

It should be understood that the field $\Sigma$ of rational functions on $V$ is the field $K\left(\xi_{1}, \xi_{2}, \cdots, \xi_{n}\right)$ generated by the nonhomogeneous coordinates. The field $\mathrm{K}\left(\eta_{0}, \eta_{1}, \cdots, \eta_{n}\right)$ is a simple transcendental extension of $\Sigma$. The field $\Sigma$ consists of all quotients $f(\eta) / g(\eta)$, where $f$ and $g$ are forms of like degree; in other words, $\Sigma$ consists of all elements of the field $\mathrm{K}\left(\eta_{0}, \eta_{1}, \cdots, \eta_{n}\right)$ which are homogeneous of degree zero (Zariski [7, p. 284]).

We have also two coordinate rings: the ring $\mathrm{K}\left[\eta_{0}, \eta_{1}, \cdots, \eta_{n}\right]$ of the homogeneous coordinates, which we have denoted by $P$, and-for a given choice of the nonhomogeneous coordinates $\xi_{i}$-the ring $\mathrm{K}\left[\xi_{1}, \xi_{2}, \cdots, \xi_{n}\right]$ which we shall denote by $\mathfrak{o}$. In order to elicit the relationship between the ideals in $\mathfrak{D}$ and the homogeneous ideals in $P$, we assume for simplicity that $\xi_{i}=\eta_{i} / \eta_{0}$ and we consider the ring $0^{*}=K\left(\eta_{0}\right)\left[\xi_{1}, \xi_{2}, \cdots, \xi_{n}\right]$. Since $\eta_{0}$ is a transcendental with respect to $\Sigma$, the two rings $\mathfrak{o}$ and $\mathfrak{o}^{*}$ are in the same relationship to each other as the two rings $P, P^{*}$ of the preceding section. Therefore the correspondence between the ideals in 0 and their extended ideals in $0^{*}$ satisfies all the relations a-e (in which $P$ and $P^{*}$ are naturally to be replaced by 0 and $0^{*}$ ). We shall denote by $M^{*}$ the class of $0^{*}$-ideals which are extensions of $\mathrm{D}$-ideals.

From the pair of rings, $0,0^{*}$ we pass to the pair of rings $0^{*}, P$. We have: $0^{*}=\mathrm{K}\left(\eta_{0}\right)\left[\eta_{1}, \eta_{2}, \cdots, \eta_{n}\right]$. The polynomial ring $\mathrm{K}\left[\eta_{0}\right]$ is a subring of $P$, and thus $\mathrm{D}^{*}$ is a quotient ring of $P$, since $\mathrm{K}\left[\eta_{0}\right]$ is at any rate closed under multiplication $\left.{ }^{1}\right)$. There is therefore a $(1,1)$ correspondence between the ideals $\mathfrak{A}^{*}$ in $\mathfrak{D}^{*}$ and those ideals $\mathfrak{A}$ in $P$ which are relatively prime to all elements of $\mathrm{K}\left[\eta_{0}\right]$, that is, which are such that $\mathfrak{A}: \alpha=\mathfrak{A}$, for all $\alpha$ in $\mathrm{K}\left[\eta_{0}\right]$. The correspondence is again that of extension and contraction: $\mathfrak{A}^{*}=\mathfrak{d}^{*} \mathfrak{A}, \mathfrak{A}=\mathfrak{A} * \cap P$. Prime or primary ideals $\mathfrak{A}$ go, respectively, into prime or primary ideals $\mathfrak{A}^{*}$.

(1) For properties of quotient rings used in the text, see Grell [1] and Krull [2, p. 18]. We recall that a quotient ring is defined as follows: if $R$ is an integral domain and if $S$ is a subset of $R$ which is closed under multiplication, then the quotients $\alpha / \beta, \alpha, \beta \in R, \beta \in S$, form a ring. This is the quotient ring $R_{S}$. A special important case is the one in which $S$ is the set-theoretic complement of a prime ideal $\mathfrak{p}$ in $R$, that is, $S=R-\mathfrak{p}$. In this case one writes $R_{\mathfrak{p}}$ instead of $R_{S}$. 
The isomorphism relations $\mathrm{c}, \mathrm{c}_{1}, \mathrm{c}_{2}, \mathrm{c}_{3}$ of the preceding section, with $P^{*}$ replaced by $0^{*}$, continue to hold $\left({ }^{2}\right)$. These are properties which hold quite generally for quotient rings.

It is immediately seen that an $\mathfrak{D}^{*}$-ideal $\mathfrak{I}^{*}$ is in the class $M^{*}$ if and only if the corresponding ideal $\mathfrak{A}$ in $P$ is homogeneous. It is clear that a homogeneous ideal is always relatively prime to any polynomial $f\left(\eta_{0}\right)$ if that polynomial contains a constant term which is different from zero. Hence, for a homogeneous ideal $\mathfrak{A}$ to be relatively prime to each element of $\mathrm{K}\left[\eta_{0}\right]$, it is necessary and sufficient that it be relatively prime to $\eta_{0}$. An equivalent condition is that no prime ideal of $\mathfrak{A}$ be a divisor of $\left(\eta_{0}\right)$. We have therefore $a(1,1)$ correspondence between the homogeneous ideals in $P$ which satisfy this last mentioned condition and the ideals in $\mathbf{v}$. This conclusion corresponds to the obvious geometrical fact that by passing to nonhomogeneous coordinates $\eta_{i} / \eta_{0}$ we lose track of all the subvarieties of $V$ which "are at infinity," that is, which lie in the hyperplane $y_{0}=0$.

Concretely, the relationship between two corresponding ideals $\mathfrak{a}$ and $\mathfrak{A}$ in $\mathfrak{D}$ and $P$, respectively, is as follows: a form $f\left(\eta_{0}, \eta_{1}, \cdots, \eta_{n}\right)$ belongs to $\mathfrak{A}$ if and only if $f\left(1, \xi_{1}, \cdots, \xi_{n}\right)$ belongs to a; a polynomial $\phi\left(\xi_{1}, \xi_{2}, \cdots, \xi_{n}\right)$ belongs to $\mathfrak{a}$, if and only if there exists in $\mathfrak{A}$ a form $f\left(\eta_{0}, \eta_{1}, \cdots, \eta_{n}\right)$ such that $f\left(1, \xi_{1}, \cdots, \xi_{n}\right)=\phi\left(\xi_{1}, \xi_{2}, \cdots, \xi_{n}\right)$.

3. The center of a valuation. Let $W$ be an irreducible subvariety of $V$. If $\xi_{1}, \xi_{2}, \cdots, \xi_{n}$ are nonhomogeneous coordinates with respect to which $W$ is at finite distance, then $W$ is given by a prime ideal $\mathfrak{p}$ in the ring $\mathbf{0}$. By the quotient ring $Q(W)$ of $W$ we mean the quotient ring $\mathfrak{o}_{\mathfrak{p}}$. If $\mathfrak{B}$ is the homogeneous prime ideal which corresponds to $W$ in the ring $P$, then it is easily seen that $Q(W)$ consists of all quotients $f(\eta) / g(\eta)$, where $f$ and $g$ are forms of like degree in $\eta_{0}, \eta_{1}, \cdots, \eta_{m}$ and where $g(\eta) \not \equiv 0(\mathfrak{P})$. This shows, incidentally, that $Q(W)$ is independent of the choice of the nonhomogeneous coordinates.

The relationship between the ideals in $\mathfrak{D}$ and the ideals in $Q(W)$ is the one described in the preceding section for general quotient rings. The $(1,1)$ correspondence is now between the ideals in $Q(W)$ and those ideals in $\mathfrak{D}$ which are relatively prime to each element $\left(^{3}\right)$ of $\mathfrak{D}-\mathfrak{p}$. An ideal in $\mathbf{v}$ satisfies this condition if and only if each prime ideal of this ideal is a multiple of $\mathfrak{p}$. This shows that by passing from the ring $\mathfrak{D}$ (or from the ring $P$ ) to the quotient ring $Q(W)$ we lose all irreducible subvarieties of $V$ which do not contain $W$. For this reason we may regard the ideal theory of $Q(W)$ as that pertaining to the "neighborhood" of $W$. An important property of the quotient ring $o_{p}$ is the

(2) However, it is to be pointed out that the set of ideals $\mathfrak{A}$ which correspond to ideals $\mathfrak{A}^{*}$ in the above correspondence is not in general closed under the operations of multiplication and addition of ideals. It is closed under the operations of intersection and quotient formation.

(3) Quite generally, given a quotient ring $R_{S}$ (see footnote 1 ), there is a $(1,1)$ correspondence between the ideals in $R_{S}$ and the ideals in $R$ which are relatively prime to each element of $S$. 
following: the non-units in $\mathfrak{o}_{\mathfrak{p}}$ form an ideal $\left({ }^{4}\right)$. This is the prime ideal in $\mathfrak{o}_{\mathfrak{p}}$ which corresponds to (that is, is the extension of) the prime ideal $\mathfrak{p}$ in $\mathfrak{D}$.

Let $v$ be a non-trivial valuation of the field $\Sigma$ over $\mathrm{K}(v(c)=0$ if $c \in \mathrm{K}$ and $c \neq 0 ; v(\omega) \neq 0$ for some $\omega$ in $\Sigma)$. We consider linear forms $l$ in $\eta_{0}, \eta_{1}, \cdots, \eta_{n}$, with coefficients in $\mathrm{K}$, such that $v\left(\eta_{i} / l\right) \geqq 0, i=0,1, \cdots, n$. If $l_{0}$ is one such form, we consider the homogeneous ideal $\mathfrak{B}$ generated by the forms $f(\eta)$ having the following property: if $f(\eta)$ is of degree $m$, then $v\left(f / l_{0}^{m}\right)>0$. The ideal $\mathfrak{B}$ is independent of $l_{0}$, since if $l_{1}$ is another linear form $l$, then $v\left(l_{1} / l_{0}\right)=0$. Moreover, $\mathfrak{B}$ is obviously a prime ideal, different from the irrelevant prime. It is also different from the zero ideal, since $v$ is a non-trivial valuation. Consequently $\mathfrak{B}$ defines an irreducible proper subvariety $W$ of $V$, of dimension at least 0 . This subvariety $W$ we call the center of the valuation $v$ (on $V$ ).

It is clear that any linear form $l$ for which $v\left(\eta_{i} / l\right) \geqq 0, i=0,1, \cdots, n$, is such that $l \neq 0$ on $W$. Conversely, if $l$ is a linear form in the $\eta$ 's and if $l \neq 0$ on $W$, then we must have: $v\left(l / l_{0}\right)=0$, whence $v\left(\eta_{i} / l\right)=v\left(\eta_{i} / l_{0}\right) \geqq 0$. Thus the linear forms $l$ which played an auxiliary role in our definition of the center $W$ of $v$, turn out simply to be those forms which do not vanish on that center.

In terms of nonhomogeneous coordinates the center $W$ is obtained as follows. Let us consider the nonhomogeneous coordinates $\xi_{i}=\eta_{i} / \eta_{0}$. Should the center $W$ be at finite distance with respect to these coordinates, we must have $\eta_{0} \neq 0$ on $W$. But then, by the remark just made, $v\left(\eta_{i} / \eta_{0}\right) \geqq 0, i=0,1, \cdots, n$, and the entire coordinate ring $\mathrm{D}$ must be contained in the valuation ring $R_{v}$ of $v$. Conversely, if $\mathrm{D} \subseteq R_{v}$, then reversing the above reasoning we see immediately that the center $W$ of $v$ is at finite distance with respect to the nonhomogeneous coordinates $\xi_{i}$. If $f\left(\eta_{0}, \eta_{1}, \cdots, \eta_{n}\right)$ is a form of degree $\nu$ and if $f=0$ on $W$, then $v\left(f / \eta_{0}^{\nu}\right)>0$, whence $v\left(f\left(1, \xi_{1}, \cdots, \xi_{n}\right)\right)>0$. Conversely, it is seen immediately that every polynomial in $\xi_{1}, \xi_{2}, \cdots, \xi_{n}$ which has positive value in $v$ arises from a form in the $\eta$ 's which vanishes on $W$. Hence the elements of $\mathrm{D}$ which have positive value in the given valuation $v$ form a prime ideal $\mathfrak{p}$ in $\mathfrak{D}$, and the center of $v$ is the irreducible subvariety of $V$ defined by the ideal $\mathfrak{p}$. This conclusion holds for any choice of the nonhomogeneous coordinates, provided the corresponding coordinate ring is contained in the valuation ring $R_{v}$.

The following characterizations of the center of a valuation are useful in applications :

Theorem 3. An irreducible subvariety $W$ of $V$ is the center of a valuation $v$ if and only if either one of the following conditions is satisfied: (1) $Q(W) \subseteq R_{v}$ and the non-units of $Q(W)$ are non-units of $R_{v}$; (2) $Q(W) \subseteq R_{v}$ and $W$ is the maximal subvariety of $V$ whose quotient ring is contained in $R_{v}$.

Proof. Suppose that $W$ is the center of $v$. If $f(\eta) / g(\eta) \in Q(W)$, where $f$ and $g$

(4) Chain theorem rings with the property that their non-units form an ideal have been called by Krull "Stellenringen" (see Krull [3]). We propose the translation: "local rings." The quotient ring of any irreducible subvariety of $V$ is a local ring. 
are forms of degree $m$, then $g(\eta) \neq 0$ on $W$. Therefore $v\left(g / l_{0}^{m}\right)=0$, while $v\left(f / l_{0}^{m}\right) \geqq 0$, and consequently $f(\eta) / g(\eta) \in R_{v}$. Moreover, if $f / g$ is a non-unit in $Q(W)$, then $f=0$ on $W$. Hence $v\left(f / l_{0}^{m}\right)>0$, and consequently $v(f / g)>0$, that is, $f / g$ is also a non-unit in $R_{v}$. This proves that condition (1) is necessary. To show the necessity and sufficiency of condition (2), let $W_{1}$ be another irreducible subvariety of $V$ with the property: $Q\left(W_{1}\right) \subseteq R_{v}$. Let $l_{0}$ be a linear form in $\eta_{0}, \eta_{1}, \cdots, \eta_{m}$ which does not vanish on $W_{1}$. Then $\eta_{i} / l_{0} \in Q\left(W_{1}\right) \subseteq R_{v}$, whence $v\left(\eta_{i} / l_{0}\right) \geqq 0, i=0,1, \cdots, n$. This shows that $l_{0} \neq 0$ on $W$. Let now $f(\eta)$ be any form in the $\eta$ 's which vanishes on $W$. If $f$ is of degree $m$, then $v\left(f / l_{0}^{m}\right)>0$, and consequently $l_{0}^{m} / f \notin R_{v}$. Since, by hypothesis, $Q\left(W_{1}\right) \subseteq R_{v}$, we have $a$ fortiori, $l_{0}^{m} / f \notin Q\left(W_{1}\right)$, whence $f=0$ on $W_{1}$. Thus we find that " $f=0$ on $W$ " implies " $f=0$ on $W_{1}$." Therefore $W_{1} \subseteq W$, and this proves our assertion.

Now it follows immediately that condition (1) is also sufficient. For, if $W_{1}$ is any proper subvariety of the center $W$ of $v$, then $\left(^{5}\right) Q\left(W_{1}\right) \subset Q(W)$ and there exist non-units in $Q\left(W_{1}\right)$ which are units in $Q(W)$ and which are therefore also units in $R_{v}$. This completes the proof of the theorem.

We shall conclude this section with two lemmas which we shall have occasion to use in the sequel.

LEммA 1. If $W$ and $W_{1}$ are irreducible subvarieties of $V$, then $W_{1} \subseteq W$ if and only if $Q\left(W_{1}\right) \subseteq Q(W)$, and $\left(^{6}\right) W_{1} \subset W$ if and only if $Q\left(W_{1}\right) \subset Q(W)$.

The proof is straightforward. If $W_{1} \subseteq W$ and if $W_{1}$ is at finite distance with respect to the nonhomogeneous coordinates $\xi_{i}$, then also $W$ is at finite distance, and the corresponding prime ideals $p_{1}$ and $p$ are such that $\mathfrak{p}_{1} \supseteq \mathfrak{p}$. Hence $\mathfrak{o}_{\mathfrak{p}_{1}} \subseteq \mathfrak{o}_{\mathfrak{p}}$. If $\mathfrak{p}_{1} \supset \mathfrak{p}$, and if $\alpha$ is an element of $\mathfrak{p}_{1}$, not in $\mathfrak{p}$, then $1 / \alpha \in \mathfrak{o}_{\mathfrak{p}}$, but $1 / \alpha \notin \mathfrak{D}_{\mathfrak{p}_{1}}$, whence $\mathfrak{D}_{\mathfrak{p}_{1}} \subset \mathfrak{o}_{\mathfrak{p}}$. Conversely, assume that $Q\left(W_{1}\right) \subseteq Q(W)$. If $\mathfrak{B}$ and $\mathfrak{P}_{1}$ are the homogeneous ideals corresponding respectively to $W$ and $W_{1}$, let $g(\eta)$ be an arbitrary form such that $g \neq 0$ on $W_{1}$. Let $f(\eta)$ be a form of the same degree as $g$, such that $f \neq 0$ on $W$. Then $f / g \in Q\left(W_{1}\right)$, and hence $f / g \in Q(W)$. This implies $g \neq 0$ on $W$, in view of the assumption that $f \neq 0$ on $W$. Hence if $g \neq 0$ on $W_{1}$ we must also have: $g \neq 0$ on $W$. This shows that $\mathfrak{B}_{1} \supseteq \mathfrak{P}$, whence $W_{1} \subseteq W$, as asserted.

LEMмA 2. If $v$ and $v_{1}$ are two valuations of $\Sigma / \mathrm{K}$, with centers $W$ and $W_{1}$, respectively, and if $v$ is composite with $v_{1}$, then $W \subseteq W_{1}$.

Proof. A valuation $v$ is composite with another valuation $v_{1}$, if $v$ is obtained by combining $v_{1}$ with a valuation $v^{\prime}$ of the residue field $\Sigma_{1}$ of $v_{1}$. The manner in which $v$ and $v^{\prime}$ are to be combined is best described in terms of the homomorphic mapping of $\Sigma$ upon the residue field of the valuation (together with the symbol $\infty$ ), a mapping which is determined by the valuation

(5) See the lemma which follows immediately.

(b) We use the symbol $\subset$ only for proper subsets. 
and which in its turn determines the valuation uniquely. Let $\tau_{1}$ be the homomorphic mapping of $\Sigma$ upon $\left(\Sigma_{1}, \infty\right)$ determined by $v_{1}$, and let $\tau^{\prime}$ be the homomorphic mapping of $\Sigma_{1}$ upon the residue field $\Sigma_{1}^{\prime}$ (and the symbol $\infty$ ) of $v^{\prime}$. Then $v$ is the valuation of $\Sigma$ determined by the homomorphic mapping $\tau=\tau_{1} \tau^{\prime}$ of $\Sigma$ onto $\left(\Sigma_{1}^{\prime}, \infty\right)$. For further details, see Krull [2, p. 112].

Now let $f(\eta)$ be a form which vanishes on $W_{1}$ and let $g(\eta)$ be a form of the same degree as $f(\eta)$ such that $g \neq 0$ on $W$ and on $W_{1}$. The quotient $\zeta=f / g$ is a non-unit of $Q\left(W_{1}\right)$. Hence, by Theorem $3, \zeta$ is also a non-unit in $R_{v_{1}}$. Consequently $\tau_{1}(\zeta)=0$, and therefore also $\tau(\zeta)=0$. Hence $\zeta$ is a non-unit in $R_{v}$, and, in view of our assumption that $g$ is not zero on $W$, this is only possible if $f=0$ on $W$, q.e.d.

4. Existence theorems for valuations with a preassigned center. If $R_{v}$ is the valuation ring of a given valuation $v$ of $\Sigma / K$ and if $\mathfrak{B}_{v}$ denotes the prime ideal of non-units of $R_{v}$, then by the dimension of $v$ is meant the degree of transcendency of the residue field $R_{v} / \mathfrak{P}_{v}$ (over $\mathrm{K}$ ). Let $W$ be the center of $v$ on $V$ and let $\Im$ denote the quotient ring $Q(W)$. By Theorem 3 we have: $\mathfrak{B}_{v} \cap \mathfrak{F}=\mathfrak{m}$, where $\mathfrak{m}$ is the ideal of non-units in $\Im$. Hence $\Im / \mathfrak{m}$ is a subring of $R_{v} / \mathfrak{B}_{v}$, and therefore the dimension of $W$ is not greater than the dimension of $v$. If $v$ is of dimension $r-1$, it is called a divisor. A divisor is of the first or of the second kind with respect to $V$, according as its center on $V$ is of dimension $r-1$ or less than $r-1$.

THEOREM 4. Given an s-dimensional irreducible subvariety $W$ of $V$, there exist valuations of center $W$, of any dimension $\rho, s \leqq \rho \leqq r-1$.

Proof. We consider first two special cases: (a) $s=r-1$; (b) $s<r-1$, $\rho=r-1$.

Case (a) $(s=r-1)$. Let $\Im^{*}$ be the integral closure of $\Im$ in $\Sigma$. The $(r-1)$ dimensional prime ideal $\mathfrak{m}$ of $\Im$ may split in $\Im^{*}$ into several prime ideals $\mathfrak{m}_{1}^{*}, \mathfrak{m}_{2}{ }^{*}, \cdots, \mathfrak{m}_{h}^{*}$, all of the same dimension $r-1$. It is well known that the quotient rings $\Im_{\mathrm{mi}_{i}^{*}}^{*}$ are valuation rings of divisors $v_{1}, v_{2}, \cdots, v_{h}$. The center of each divisor $v_{i}$ is our preassigned $W$, and in this fashion all the divisors of center $W$ are obtained.

Case (b) $(s<r-1, \rho=r-1)$. Assuming that $W$ is at finite distance with respect to the nonhomogeneous coordinates $\xi_{i}$, let $\boldsymbol{D}$ denote, as usual, the ring of these coordinates, and let $p$ be the prime ideal of $W$ in 0 . Let $\omega_{1}, \omega_{2}, \cdots, \omega_{m}$ be a base of the ideal $\mathfrak{p}$. We select one element among these $m$ elements $\omega_{i}$ and we denote it by $\omega$. We pass to the ring $\mathfrak{D}^{\prime}=\mathfrak{D}\left[\omega_{1} / \omega, \omega_{2} / \omega, \cdots, \omega_{m} / \omega\right]$, and we first prove the following lemma:

LEMMA 3. For at least one mode of selecting the element $\omega$ among the elements $\omega_{1}, \omega_{2}, \cdots, \omega_{m}$, the following relation will be satisfied: $\mathfrak{o}^{\prime} \cdot \mathfrak{p} \bigcap_{\mathfrak{D}}=\mathfrak{p}$.

Proof of the lemma. Since $\omega_{i}=\left(\omega_{i} / \omega\right) \cdot \omega \in \mathrm{o}^{\prime} \cdot \omega$, it follows that the ideal $\mathfrak{o}^{\prime} \cdot \mathfrak{p}$ coincides with the principal ideal $\mathfrak{D}^{\prime} \cdot \omega$. Now let us suppose that 
$\mathfrak{D}^{\prime} \cdot \omega \cap \mathfrak{D} \neq \mathfrak{p}$, and let us see what restriction, if any, this assumption imposes on the element $\omega$. Let $\zeta$ be an element of $\mathfrak{D}$, which belongs to $\mathfrak{D}^{\prime} \cdot \boldsymbol{\omega}$ but not to $p$. We will have then: $\zeta=\omega \cdot f\left(\omega_{1} / \omega, \omega_{2} / \omega, \cdots, \omega_{m} / \omega\right)$, where $f(z)=f\left(z_{1}, z_{2}, \cdots, z_{m}\right) \in \mathfrak{o}\left[z_{1}, z_{2}, \cdots, z_{m}\right]$. If $\nu$ is the degree of $f$, the above expression for $\zeta$ leads to a relation of the form: $\zeta \cdot \omega^{\nu-1}=\phi\left(\omega_{1}, \omega_{2}, \cdots, \omega_{m}\right)$, where $\phi$ is a form of degree $\nu$, with coefficients in $\mathfrak{D}$. This relation implies that $\zeta \cdot \omega^{\nu-1}$ is in $\mathfrak{p}^{\nu}$. Since $\zeta \not \equiv 0(\mathfrak{p})$, we conclude that $\mathfrak{p}^{\nu}: \omega^{\nu-1}$ is a proper divisor of $\mathfrak{p}$.

If our lemma is false, then for each element $\omega_{i}, i=1,2, \cdots, m$, there must exist an integer $\nu_{i}$ such that $p^{\nu_{i}}: \omega_{i}^{\nu_{i}-1} \supset \mathfrak{p}$. If $\sigma=$ maximum $\left(\nu_{1}, \nu_{2}, \cdots, \nu_{m}\right)$, then we will have $\mathfrak{p}^{\sigma}: \omega_{i}^{\sigma-1} \supset \mathfrak{p}, i=1,2, \cdots, m$. Hence we have also $\mathfrak{p}^{\sigma+\rho}: \mathfrak{p}^{\rho} \omega_{i}^{\sigma-1} \supset \mathfrak{p}$, for any integer $\rho$. Now if $q=(\sigma-2) \cdot m+1$, then it is clear that $\left(\mathfrak{p}^{p} \omega_{1}^{\sigma-1}, \mathfrak{p}^{\rho} \omega_{2}^{\sigma-1}, \cdots, \mathfrak{p}^{\rho} \omega_{m}^{\sigma-1}\right)=\mathfrak{p}^{q}$, if $\rho=q-\sigma+1$. Therefore the quotient $\mathfrak{p}^{q+1}: \mathfrak{p}^{q}$ is the intersection of the ideals $\mathfrak{p}^{q+1}: \mathfrak{p}^{q-\sigma+1} \omega_{i}^{\sigma-1}, i=1,2, \cdots, m$. But each of these $m$ ideals is, by hypothesis, a proper divisor of $p$. Hence also $\mathfrak{p}^{q+1}: \mathfrak{p}^{q}$ is a proper divisor, and this is impossible since $\left(^{7}\right) \mathfrak{p}^{q+1}: \mathfrak{p}^{q}=\mathfrak{p}$. Our lemma is thus proved.

We therefore may assume that $\mathfrak{o}^{\prime} \cdot \mathfrak{p} \cap \mathfrak{v}=\mathfrak{p}$. This implies at any rate that the ideal $\boldsymbol{o}^{\prime} \cdot \omega$ is not the unit ideal, whence its minimal prime ideals are all $(r-1)$-dimensional. The relation $\mathfrak{o}^{\prime} \cdot \mathfrak{p} \cap \mathfrak{D}=\mathfrak{p}$ also implies, and is in fact equivalent to, the assertion that at least one minimal prime ideal of $\boldsymbol{o}^{\prime} \cdot \omega$ must contract to $\mathfrak{p}$. Let $\mathfrak{p}^{\prime}$ be such a minimal prime.

Now let $V^{\prime}$ be the projective model whose general point (in nonhomogeneous coordinates) is $\left(^{8}\right)\left(\xi_{1}, \xi_{2}, \cdots, \xi_{n}, \omega_{1} / \omega, \omega_{2} / \omega, \cdots, \omega_{m} / \omega\right)$, so that $\mathrm{D}^{\prime}$ is the ring of the nonhomogeneous coordinates of the general point of $V^{\prime}$. Let $W^{\prime}$ be the $(r-1)$-dimensional subvariety of $V^{\prime}$ which is defined by the prime ideal $\mathfrak{p}^{\prime}$. By the preceding case (a) there exists a $(r-1)$-dimensional valuation whose center on $V^{\prime}$ is $W^{\prime}$. Since $\mathfrak{p}^{\prime} \cap_{\mathfrak{p}}=\mathfrak{p}$, it follows that the center of $v$ on $V$ is $W$. This establishes our theorem in the case under consideration.

To prove the theorem in the general case, $s<r-1, s \leqq \rho<r-1$, we shall keep $s$ and $\rho$ fixed and we shall proceed by induction with respect to $r$, since, by the case (b), the theorem is true if $r=\rho+1$. We consider an $(r-1)$-dimensional irreducible subvariety $W_{1}$ of $V$ which contains $W$ and we denote by $v_{1}$ a divisor of center $W_{1}$. The residue field $\Sigma_{1}^{*}$ of $v_{1}$ is a finite algebraic extension of the field $\Sigma_{1}$ of rational functions on $W_{1}$. By our induction there exists a valuation $v^{\prime}$ of $\Sigma_{1}$, of dimension $\rho$, whose center on $W_{1}$ is $W$. This valuation $v^{\prime}$ has at least one extension $v^{*}$ in $\Sigma_{1}^{*}$. Compounding $v_{1}$ with $v^{*}$ we get a composite valuation $v$ of $\Sigma$, of dimension $\rho$, whose center is $W$. This completes the proof of our theorem.

(7) Let $\mathfrak{p}^{q+1}: \mathfrak{p}^{q}=\mathfrak{A}$. We have then: $\mathfrak{A} \mathfrak{p}^{q} \equiv 0\left(\mathfrak{p}^{q+1}\right)$. On the other hand $\mathfrak{p} \subseteq \mathfrak{A}$, whence $\mathfrak{A} \cdot \mathfrak{p}^{q} \supseteq \mathfrak{p}^{q+1}$. Consequently $\mathfrak{A} \cdot \mathfrak{p}^{q}=\mathfrak{p}^{q+1}=\mathfrak{p} \cdot \mathfrak{p}^{q}$. From this it follows (see Krull $[2, \mathfrak{p} .36]$ ) that $\mathfrak{A}$ and $\mathfrak{p}$ have the same radical. Since $\mathfrak{A} \supseteq \mathfrak{p}$, it follows that $\mathfrak{A}=\mathfrak{p}$.

${ }^{8}$ ) Comparison with section 11 will show that our $V^{\prime}$ is the transform of $V$ by a monoidal transformation of center $W$. 
The preceding proof does not give an adequate idea of the totality of all valuations having a preassigned center. The valuations obtained in the course of the proof are special in the sense that if their dimension is $\rho$ then the rank of their value group is $r-\rho$. The following theorem gives more information about the arbitrary elements which can be assigned in the construction of valuations with a given center $\left(^{9}\right)$ :

THEOREM 5. Given an arbitrary descending chain $\left({ }^{10}\right) W_{0} \supseteq W_{1} \supseteq \ldots \supseteq W_{\sigma-1}$ of irreducible subvarieties of $V$ and given any set of integers $\rho_{0}, \rho_{1}, \cdots, \rho_{\sigma-1}$ such that $r-1 \geqq \rho_{0}>\rho_{1}>\cdots>\rho_{\sigma-1}, \rho_{i} \geqq$ dimension of $W_{i}$, there exists $a$ sequence of valuations $v_{0}, v_{1}, \cdots, v_{\sigma-1}$ such that: (1) $v_{i}$ is of dimension $\rho_{i}$, of rank $i+1$, and its center is $W_{i}$; (2) $v_{i}$ is compounded with $v_{i-1}$.

We first prove the theorem in the following two special cases: (a) $\sigma=1$, $\rho_{0}=s=$ dimension of $W_{0}$; (b) $\sigma=1, \rho_{0}>s$.

(a) $\sigma=1, \rho_{0}=s=$ dimension $W_{0}$. In this case we have to prove the existence of a rank 1 valuation, of dimension $s$, whose center is a given $s$-dimensional irreducible subvariety $W_{0}$ of $V$. We shall use nonhomogeneous coordinates $\xi_{1}, \xi_{2}, \cdots, \xi_{n}$ with respect to which $W_{0}$ is at finite distance, so that $W_{0}$ is given by a prime ideal $\mathfrak{p}$ in the ring $\mathfrak{D}$ of these coordinates. We then adjoin to the ground field $s$ elements of $\mathfrak{o}$ which are algebraically independent on $W$, that is, algebraically independent mod $\mathfrak{p}$. In this fashion we achieve a reduction to the case $s=0$, so that we may assume that $W_{0}$ is a point, say $A$. It is also permissible to assume ${ }^{11}$ ) that $\xi_{r+1}, \xi_{r+2}, \cdots, \xi_{n}$ are

(9) Which ordered groups can be preassigned as value groups for valuations of fields of algebraic functions, is a question which has been solved completely by S. MacLane and O. F. G. Schilling in their paper [4].

(10) Note that we do not assume that the chain is strictly descending, that is, that each $W_{i}$ is a proper subvariety of $W_{i-1}$.

(11) In the case of infinite ground fields, or of ground fields with "sufficiently many" elements, this assumption can always be realized, in view of the usual proof of Emmy Noether's normalization theorem, by first subjecting the nonhomogeneous coordinates $\xi_{1}, \xi_{2}, \cdots, \xi_{n}$ to a linear transformation with "non-special" coefficients in $\mathbf{K}$. In the case of finite ground fields this is no longer true. However, it will be proved in II.2 that there always exists in the ring $\mathbf{K}\left[\xi_{1}, \xi_{2}, \cdots, \xi_{n}\right]$ a set of $r$ algebraically independent elements $\zeta_{1}, \zeta_{2}, \cdots, \zeta_{r}$ such that the ring is integrally dependent on the ring $K\left[\zeta_{1}, \zeta_{2}, \cdots, \zeta_{r}\right]$. We may then simply include the elements $\zeta_{i}$ among the elements $\xi_{i}$; which does not change the ring of nonhomogeneous coordinates, and we may then proceed as in the text.

We could also proceed in the following fashion. We first pass to the field $K^{\prime}$ which is generated over $K$ by the coefficients of the linear transformation on the $\xi_{i}$ mentioned above, and we consider an extension field $\Sigma^{\prime}=K^{\prime} \Sigma$ of $\Sigma$. The field $K^{\prime}$ may be assumed to be an algebraic extension of $\mathrm{K}$. We obtain a new variety $V^{\prime}$ over $\mathbf{K}^{\prime}$, with the same general point $\left(\xi_{1}, \xi_{2}, \cdots, \xi_{n}\right)$ as $V$. The original subvariety $W_{0}$ splits on $V^{\prime}$ into at most a finite number of varieties, all of the same dimension as $W_{0}$. If $W_{0}^{\prime}$ is one of them, the proof given in the text leads to a valuation $v^{\prime}$ of $\Sigma^{\prime}$ of dimension $s$ and rank 1 , with center $W_{0}^{\prime}$. The valuation $v$ of $\Sigma$ induced by $v^{\prime}$ will have center $W_{0}$, dimension $s$ and rank 1 . 
integrally dependent on $\mathrm{K}\left[\xi_{1}, \xi_{2}, \cdots, \xi_{r}\right]$. Let $A^{\prime}$ be the projection( $\left.{ }^{12}\right)$ of the point $A$ into the linear space of the $r$ independent variables $\xi_{1}, \xi_{2}, \cdots, \xi_{r}$. Let $v^{\prime}$ be a zero-dimensional and rank 1 valuation of the field $\mathrm{K}\left(\xi_{1}, \xi_{2}, \cdots, \xi_{r}\right)$ whose center in the above linear space is the point $\left({ }^{13}\right) A^{\prime}$. We denote, as usual, by $R_{v^{\prime}}$ the valuation ring of $v^{\prime}$, and by $A_{1}, A_{2}, \cdots, A_{h}$ the other points of $V$, at finite distance and different from $A$, which project into $A^{\prime}$. We can find an element $\omega$ in $\mathfrak{D}$ such that $\omega=0$ at $A, \omega \neq 0$ at $A_{i}, i=1,2, \cdots, h$. Let

$$
\omega^{m}+a_{1}\left(\xi_{1}, \xi_{2}, \cdots, \xi_{r}\right) \omega^{m-1}+\cdots+a_{m}\left(\xi_{1}, \xi_{2}, \cdots, \xi_{r}\right)=0
$$

be the irreducible equation of integral dependence for $\omega$ over the ring $\mathrm{K}\left[\xi_{1}, \xi_{2}, \cdots, \xi_{r}\right]$. Since $\omega=0$ at $A$, we must have $a_{m}=0$ at $A^{\prime}$. Hence $a_{m}$ is a non-unit in $R_{v^{\prime}}$. We assert that $R_{v^{\prime}}[\omega]$ is a proper ring (that is, is not a field). We prove this by showing that $\omega$ is a non-unit in this ring. For suppose that $\omega$ is a unit in $R_{v^{\prime}}[\omega]$. Then we would have: $1=\omega \cdot g(\omega)$, where $g(\omega)$ is a polynomial with coefficients in $R_{v^{\prime}}$. Using the above equation of integral dependence and observing that the coefficients $a_{i}$ are polynomials, hence are elements of $R_{v^{\prime}}$, we can reduce the degree of $g(\omega)$. We thus find a new relation of the form: $1=\omega\left(b_{0} \omega^{m-1}+b_{1} \omega^{m-2}+\cdots+b_{m-1}\right)$, where $b_{i} \in R_{v^{\prime}}$. Comparing this relation with the above relation of integral dependence, we conclude that $b_{0}=-1 / a_{m}$, a contradiction, since $a_{m}$ is a non-unit in $R_{v^{\prime}}$.

Since $\omega$ is a non-unit in $R_{v}$ [ $[\omega]$, there exists at least one valuation $v$ of $\Sigma$ such that $R_{v} \supseteq R_{v^{\prime}}[\omega]$ and such that $\omega$ is a non-unit( $\left.{ }^{14}\right)$ in $R_{v}$. Since $v^{\prime}$ is of rank $1, R_{v^{\prime}}$ is a maximal subring of $\mathrm{K}\left(\xi_{1}, \xi_{2}, \cdots, \xi_{r}\right)$. Hence $R_{v} \cap \mathrm{K}\left(\xi_{1}, \xi_{2}, \cdots, \xi_{r}\right)$ $=R_{v^{\prime}}$, and therefore the valuation $v$ is an extension of $v^{\prime}$ and has the same rank and the same dimension as $v^{\prime}$, that is, rank 1 and dimension 0 . The center of $v$ on $V$ must be a point at finite distance (since $\xi_{1}, \xi_{2}, \cdots, \xi_{r} \in R_{v} \subseteq R_{v}$ and hence $\mathfrak{D} \subseteq R_{v}$, for $R_{v}$ is integrally closed), and this point must project into the point $A^{\prime}$. The center cannot be any of the points $A_{1}, A_{2}, \cdots, A_{h}$, since $\omega \neq 0$ at $A_{i}$ and this implies that $\omega$ is a unit in the quotient ring $Q\left(A_{i}\right)$, while, as we have just seen, $\omega$ is a non-unit in $R_{v}$ (compare with Theorem 3 ). Hence the center of $v$ is the point $A$, q.e.d.

(b) Let now $\sigma=1, \rho>s$. We refer to the case (b) of the proof of Theorem 4 , where we identify the variety $W$ with our present variety $W_{0}$. We had there an $(r-1)$-dimensional prime ideal $\mathfrak{p}^{\prime}$ in $\mathfrak{v}^{\prime}$ such that $\mathfrak{p}^{\prime} \cap \mathfrak{v}=\mathfrak{p}$. From the existence of an $(r-1)$-dimensional prime ideal in $\mathfrak{o}^{\prime}$ which contracts to $\mathfrak{p}$ follows immediately the existence of prime ideals in $\mathfrak{b}^{\prime}$ of any dimension $\rho$,

${ }^{(2)}$ By that we mean that $A^{\prime}$ is the point which is defined by the contraction of the prime ideal $\mathfrak{p}$ in the polynomial ring $\mathbf{K}\left[\xi_{1}, \xi_{2}, \cdots, \xi_{r}\right]$.

(13) The existence of $v^{\prime}$ is proved in the joint paper by MacLane and Schilling [4].

(14) This is implied by the fundamental theorem on principal orders which states that an integrally closed integral domain (not a field) is the intersection of the valuation rings which contain it (see Krull [2, p. 111]). It is necessary only to observe that the integral closure of a proper ring is also a proper ring. For if a ring $R$ is proper, it contains a non-unit $\alpha$, and it is seen immediately that $1 / \alpha$ cannot be integrally dependent on $R$. 
$r-1 \geqq \rho \geqq s$, which contract to $\left.{ }^{15}\right) \mathfrak{p}$. Let $\mathfrak{p}^{\prime \prime}$ be such a prime ideal and let $W^{\prime \prime}$ be the corresponding irreducible $\rho$-dimensional subvariety of $V^{\prime}$. By the preceding case (a), there exists a valuation $v$, of rank 1 and of dimension $\rho$, whose center on $V^{\prime}$ is $W^{\prime \prime}$. Since $\mathfrak{p}^{\prime \prime} \cap \mathfrak{v}=\mathfrak{p}$, the center of $v$ on $V$ is $W$, q.e.d.

To prove our theorem in the general case, we first prove this lemma:

LEMma 4. If $W$ and $W_{1}$ are irreducible subvarieties of $V$ such that $W \subset W_{1} \subset V$ and if $v_{1}$ is a valuation of center $W_{1}$, then there exists a valuation $v$ of center $W$, which is compounded with $v_{1}$.

Proof. Let $\Sigma_{1}$ be the residue field of the valuation $v_{1}$ and let $\tau_{1}$ denote the homomorphic mapping of $\Sigma$ onto $\left(\Sigma_{1}, \infty\right)$ defined by $v_{1}$. Since $Q(W) \subset Q\left(W_{1}\right)$ (Lemma 1, I.3) and $Q\left(W_{1}\right) \subseteq R_{v_{1}}$, it follows that if we put $\Im=Q(W)$, then $\tau_{1} \cdot \Im \subseteq \Sigma_{1}$. The elements of $\Im$ which are mapped into zero under $\tau_{1}$ are nonunits in $Q\left(W_{1}\right)$. Since $W$ is a proper subvariety of $W_{1}$, there are non-units in $Q(W)$ which are units in $Q\left(W_{1}\right)$. Hence $\tau_{1} \Im$ is a proper ring. There exists then at least one valuation $v^{\prime}$ of $\Sigma_{1}$ such that $R_{v^{\prime}} \supseteq \tau_{1}$. $\Im$. Let $v_{2}$ denote the valuation obtained by compounding $v$ with $v^{\prime}$, and let $W_{2}$ be the center of $v_{2}$. By Lemma 2 , I.3, we have $W_{2} \subseteq W_{1}$. Since $R_{v^{\prime}} \supseteq \tau_{1} \cdot \Im$, it follows that $R_{v_{2}} \supseteq \Im$, whence, by Theorem 3 (I.3), we have $W \subseteq W_{2}$. If $W_{2}=W$, then our lemma is proved $\left(v=v_{2}\right)$. If, however, $W$ is a proper subvariety of $W_{2}$, then we replace $v_{1}$ and $W_{1}$ by $v_{2}$ and $W_{2}$ and we repeat the above procedure. Since $v_{2}$ is of smaller dimension than $v_{1}$, this process cannot continue indefinitely, q.e.d.

We now are in position to complete the proof of the theorem in the general case. Since by the special cases (a) and (b) treated above the theorem is true in the case $\sigma=1$, we assume that the theorem is true for $\sigma=h-1$ and we proceed to prove the theorem for $\sigma=h$. We can therefore assume the existence of the valuation $v_{0}, v_{1}, \cdots, v_{\sigma-2}$ and we have only to prove the existence of $v_{\sigma-1}$. The valuation $v_{\sigma-2}$ is of dimension $\rho_{\sigma-2}$ and its center is $W_{\sigma-2}$. We have to prove the existence of a valuation $v_{\sigma-1}$, of dimension $\rho_{\sigma-1}$, which is compounded with $v_{\sigma-2}$, is of rank one higher than the rank of $v_{\sigma-2}$ and has center $W_{\sigma-1}$. For simplicity we shall denote $W_{\sigma-2}, W_{\sigma-1}, \rho_{\sigma-2}, \rho_{\sigma-1}$ and $v_{\sigma-2}$ by $W_{1}, W, \rho_{1}, \rho$ and $v_{1}$, respectively, so that we have now:

$$
W_{1} \supseteq W, \rho_{1}>\rho \geqq \text { dimension } W ; \rho_{1} \geqq \text { dimension } W_{1} \text {. }
$$

We first provide ourselves with a projective model $V^{\prime}$ on which the center $W_{1}^{\prime}$ of $v_{1}$ is exactly of dimension $\left({ }^{16}\right) \rho_{1}$. We then consider, for auxiliary purposes,

(15) Let $\alpha_{1}, \alpha_{2}, \cdots, \alpha_{s}$ be $s$ elements of $\mathfrak{D}$ which are algebraically independent modulo $\mathfrak{p}$ and let us take as new ground field the field $\mathrm{K}_{1}=\mathrm{K}\left(\alpha_{1}, \alpha_{2}, \cdots, \alpha_{s}\right)$. If $\mathfrak{o}_{1}=\mathrm{K}_{1} \cdot \mathfrak{v}, \mathfrak{o}_{1}^{\prime}=\mathrm{K}_{1} \cdot \mathfrak{o}^{\prime}$, $\mathfrak{D}_{1} \cdot \mathfrak{p}=\mathfrak{p}_{1}, \mathfrak{D}_{1}^{\prime} \cdot \mathfrak{p}^{\prime}=\mathfrak{p}_{1}^{\prime}$, then, over $K_{1}, \mathfrak{p}_{1}$ is zero-dimensional, $\mathfrak{p}_{1}^{\prime}$ is $(r-1-s)$-dimensional, and $\mathfrak{p}_{1}^{\prime} \cap \mathfrak{o}_{1}=\mathfrak{p}_{1}$. Let $\mathfrak{p}_{1}^{\prime \prime}$ be any $(\rho-s)$-dimensional prime divisor of $\mathfrak{p}_{1}^{\prime}$, and let $\mathfrak{p}_{1}^{\prime \prime} \cap \mathfrak{o}^{\prime}=\mathfrak{p}^{\prime \prime}$. Then $\mathfrak{p}^{\prime \prime}$ is of dimension $\rho$, over $\mathrm{K}$, and $\mathfrak{p}^{\prime \prime} \cap \mathfrak{D}=\mathfrak{p}$.

(16) As nonhomogeneous coordinates of such a model $V^{\prime}$ we may take any finite set of generators of $\Sigma$ which belong to $R_{v_{1}}$ and such that $\rho_{1}$ of these generators have algebraically independent residues in the residue field of $v_{1}$. 
an arbitrary valuation $v$ which is compounded with $v_{1}$ and whose center on $V$ is $W$ (Lemma 4 ; if $W_{1}=W$, we put $v=v_{1}$ ). Let $W^{\prime}$ denote the center of $v$ on $V^{\prime}$, whence $W^{\prime} \subseteq W_{1}^{\prime}$ (Lemma 2, I.3). We next select nonhomogeneous coordinates $\xi_{1}, \xi_{2}, \cdots, \xi_{n}$ for the general point of $V$ and nonhomogeneous coordinates $\zeta_{1}, \zeta_{2}, \cdots, \zeta_{m}$ for the general point of $V^{\prime}$ in such a fashion that $W$ and $W^{\prime}$ be at finite distance with respect to these coordinates. Finally we denote by $V^{*}$ the projective model whose general point is $\left(\xi_{1}, \xi_{2}, \cdots, \xi_{n}\right.$, $\left.\zeta_{1}, \zeta_{2}, \cdots, \zeta_{m}\right)$, and we denote by $\mathfrak{D}, \mathfrak{o}^{\prime}$ and $\mathfrak{o}^{*}$, respectively, the corresponding rings $\mathrm{K}[\xi], \mathrm{K}[\zeta], \mathrm{K}[\xi, \zeta]$ of the nonhomogeneous coordinates.

Let $W^{*}$ and $W_{1}^{*}$ be the centers on $V^{*}$ of the valuations $v$ and $v_{1}$, respectively. Since $W$ and $W^{\prime}$ are at finite distance, we have: $\mathfrak{D} \subseteq R_{v}, \mathfrak{o}^{\prime} \subseteq R_{v}$, and, by a stronger reason, $\mathrm{o} \subseteq R_{v_{1}}, \mathrm{o}^{\prime} \subseteq R_{v_{1}}$. Therefore $\mathrm{o}^{*} \subseteq R_{v}, \mathrm{o}^{*} \subseteq R_{v_{1}}$, and consequently $W^{*}$ and $W_{1}^{*}$ are at finite distance. Let $\mathfrak{p}, \mathfrak{p}_{1} ; \mathfrak{p}^{\prime}, \mathfrak{p}_{1}^{\prime} ; \mathfrak{p}^{*}, \mathfrak{p}_{1}^{*}$ denote the prime ideal of $W, W_{1} ; W^{\prime}, W_{1}^{\prime} ; W^{*}, W_{1}^{*}$ in $\mathfrak{D}, \mathfrak{D}^{\prime}$ and $\mathfrak{o}^{*}$ respectively. It is clear that:

$$
\mathfrak{p}^{*} \cap \mathfrak{D}=\mathfrak{p}, \quad \mathfrak{p}_{1}^{*} \cap \mathfrak{o}=\mathfrak{p}_{1} .
$$

Moreover $W^{*} \subseteq W_{1}^{*}$. The above relations show that any valuation of center $W^{*}$ on $V^{*}$ has $W$ as center on $V$. We also point out that $V^{*}$ shares with $V^{\prime}$ the property that the center of $v_{1}$ on that variety is exactly of dimension $\rho_{1}$. This follows immediately from the relation: $\mathfrak{p}_{1}^{*} \cap \mathfrak{o}^{\prime}=\mathfrak{p}_{1}^{\prime}$. The variety $V^{\prime}$ and the auxiliary valuation $v$ have now served their purpose and will not be used any more.

(1) Suppose first that $W^{*}$ is of dimension at most $\rho$. By the special case $\sigma=1$, we can find a $\rho$-dimensional, rank 1 valuation of the field of rational functions on $W_{1}^{*}$, having $W^{*}$ as center. This valuation has at least one extension in the residue field of the valuation $v_{1}$. Let $v_{2}$ be such an extension. Since the residue field of $v_{1}$ is an algebraic extension of the field of rational functions on $W_{1}^{*}$, it follows that also $v_{2}$ is of rank 1 and dimension $\rho$. Compounding $v_{1}$ with $v_{2}$, we get a valuation $v$ of $\Sigma$, of dimension $\rho$, of rank one higher than $v_{1}$. Its center on $V^{*}$ is $W^{*}$, hence its center on $V$ is $W$. This valuation $v$ is the valuation $v_{\sigma-1}$, whose existence we have claimed in our theorem. (2) Suppose now that $W^{*}$ is of dimension greater than $\rho$. Since $p^{*} \cap \mathfrak{p}=\mathfrak{p}$ and since $\mathfrak{p}$ is of dimension at most $\rho$, it follows that we can find in $\mathfrak{D}^{*}$ a prime $\rho$-dimensional ideal which divides $\mathfrak{p}^{*}$ and which likewise contracts $\left({ }^{17}\right)$ to $\mathfrak{p}$. This prime ideal defines a $\rho$-dimensional irreducible subvariety $W_{0}^{*}$ of $V^{*}$ which we can use with the same effect instead of $W^{*}$, since the two essential conditions: (1) $W_{0}^{*} \subseteq W_{1}^{*},(2)$ every valuation of center $W_{0}^{*}$ has $W$ as center on $V$, are still satisfied. But now $W_{0}^{*}$ has dimension $\rho$, and we have therefore the case (1) just considered. This completes the proof of Theorem 5.

(17) See footnote 15. 
Part II. General theORY OF BIRATIONAL CORRESPONDENCES

1. Valuation-theoretic definition of a birational correspondence. Let $V$ and $V^{\prime}$ be two birationally equivalent $r$-dimensional irreducible algebraic varieties. The two varieties can be regarded as projective models of one and the same field $\Sigma$ of algebraic functions $\left({ }^{18}\right)$, and if they are so regarded there arises a well defined correspondence between the irreducible subvarieties of $V$ (of all possible dimension from 0 to $r-1$ inclusive) and the irreducible subvarieties of $V^{\prime}$. It is the birational correspondence between $V$ and $V^{\prime}$, or the birational transformation of $V$ into $V^{\prime}$. This correspondence, which we shall denote by $T$, is defined as follows $\left({ }^{19}\right)$ :

Definition 1. Two irreducible subvarieties $W$ and $W^{\prime}$ of $V$ and $V^{\prime}$ respectively (not necessarily of the same dimension) correspond to each other (in symbols: $\left.T(W)=W^{\prime}, T^{-1}\left(W^{\prime}\right)=W\right)$, if there exists a valuation $v$ of the field $\Sigma$ such that the center of $v$ on $V$ is $W$ and the center of $v$ on $V^{\prime}$ is $W^{\prime}$.

Note that this definition retains its full meaning also when $V$ and $V^{\prime}$ are coincident varieties (as varieties in the projective space). In this case we deal with an automorphism $\tau$ of $\Sigma$ and we have a birational transformation of $W$ into itself. It is only necessary to regard the two coincident varieties $V$ and $V^{\prime}$ as distinct projective models of $\Sigma$, in the sense that the general point of $V$ is $\left(\xi_{1}, \xi_{2}, \cdots, \xi_{n}\right)$ and the general point of $V^{\prime}$ is $\left(\tau \xi_{1}, \tau \xi_{2}, \cdots, \tau \xi_{n}\right)$.

From the results of I.3 and I.4 we deduce immediately the following properties of a birational correspondence:

A. Given $W \subset V$, there exists at least one $W^{\prime} \subset V^{\prime}$ such that $T(W)=W^{\prime}$ (Theorem 4, I.4).

B. If $W \subseteq W_{1} \subset V$ and $W_{1}^{\prime}=T\left(W_{1}\right)$, there exists a $W^{\prime}$ such that $W^{\prime}=T(W)$ and $W^{\prime} \subseteq W_{1}^{\prime}$. In particular, if to $W_{1}$ there corresponds a point $P^{\prime}$ on $V^{\prime}$, then $P^{\prime}$ corresponds to each point of $W_{1}$.

A birational correspondence is, generally speaking, not a $(1,1)$ correspondence. There may very well exist varieties $W$ on $V$ such that $T$ is not single-valued at $W$, that is, such that to $W$ there correspond more than one subvariety of $V^{\prime}$. Similarly for $T^{-1}$ and $V^{\prime}$. These varieties $W$ are exceptional in the sense that they lie on algebraic subvarieties of $V$ (see Theorem 15, II.9). The analysis of these exceptions to the $(1,1)$ character of a birational correspondence is the main goal of our study. At this stage, however, we wish

(18) The fields $\Sigma, \Sigma^{\prime}$ of rational functions on $V$ and $V^{\prime}$, respectively, are isomorphic over $\mathbf{K}$. When we say that $V$ and $V^{\prime}$ are projective models of one and the same field we imply that the fields $\Sigma, \Sigma^{\prime}$ have been identified. The identification is determined to within an automorphism of $\Sigma$. When we speak of a birational correspondence we refer to a fixed identification of the two fields.

(19) From now on irreducible subvarieties of $V$ shall be denoted by the letter $W$, with or without subscripts. Similarly $W^{\prime}, W_{1}^{\prime}, W_{i}^{\prime}$ and so on shall always denote irreducible subvarieties of $V^{\prime}$. 
to give a very simple but important criterion for the uniqueness of $T(W)$ when $W$ is given :

Theorem 6. If $T(W)=W^{\prime}$ and if $Q\left(W^{\prime}\right) \subseteq Q(W)$, then $W^{\prime}$ is the only subvariety of $V^{\prime}$ which corresponds to $W$.

Proof. By hypothesis, there exists a valuation $v_{1}$ whose center on $V$ is $W$ and whose center on $V^{\prime}$ is $W^{\prime}$. Let $v$ be an arbitrary valuation of center $W$. We have $R_{v} \supseteq Q(W)$, whence $R_{v} \supseteq Q\left(W^{\prime}\right)$. Every non-unit of $Q\left(W^{\prime}\right)$ is a nonunit of $R_{v_{1}}$ (Theorem 3, I.3), hence it is also a non-unit of $Q(W)$. We conclude that every non-unit of $Q\left(W^{\prime}\right)$ is a non-unit in $R_{v}$, and our theorem follows from Theorem 3.

2. The birational correspondence between $V$ and a derived normal model $\bar{V}$. In our paper [7] we have proved that from any irreducible algebraic variety $V$ it is possible to pass to what we have called a derived normal model $\bar{V}$ of $V$. That proof dealt only with algebraically closed ground fields of characteristic zero. To extend the proof to arbitrary ground fields additional considerations are necessary.

First of all we shall need an extension of the normalization theorem of Emmy Noether to finite ground fields. Given a finite integral domain $K\left[\xi_{1}, \xi_{2}, \cdots, \xi_{n}\right]$ of degree of transcendency $r$ over an infinite ground field $\mathrm{K}$, the Noether normalization theorem states that there exist $r$ linear combinations $\xi_{i}^{\prime}=\sum_{j=1}^{n} c_{i j} \xi_{j}, i=1,2, \cdots, r$, with coefficients in $K$, which are algebraically independent over $K$ and which are such that $\xi_{1}, \xi_{2}, \cdots, \xi_{n}$ are integrally dependent on $\xi_{1}^{\prime}, \xi_{2}^{\prime}, \ldots, \xi_{r}^{\prime}$. This theorem, as it stands, is not generally true when $K$ is a finite field. In this case we can still assert that elements such as $\xi_{1}^{\prime}, \xi_{2}^{\prime}, \cdots, \xi_{r}^{\prime}$ can be found in the ring $K\left[\xi_{1}, \xi_{2}, \cdots, \xi_{n}\right]$, provided we drop the condition that these elements be linear in the $\xi$ 's. The proof of this assertion, as given below, was communicated orally to me by Irvin Cohen.

We shall first consider an homogeneous integral domain $\mathfrak{D}^{*}=K\left[\eta_{0}, \eta_{1}\right.$, $\left.\cdots, \eta_{n}\right]$, of degree of transcendency $r+1$, that is, one whose generating elements $\eta_{i}$ are the homogeneous coordinates of the general point of an $r$-dimensional variety $V$.

(a) If the ideal $\left(\eta_{1}, \eta_{2}, \cdots, \eta_{n}\right)$ in $0^{*}$ is irrelevant, then $\eta_{0}$ is integrally dependent on $\eta_{1}, \eta_{2}, \cdots, \eta_{n}$. For the hypothesis implies that the point $y_{0}=1, y_{1}=y_{2}=\cdots=y_{n}=0$ is not on $V$, and hence there must exist a form $f\left(y_{0}, y_{1}, \cdots, y_{n}\right)$ such that $f\left(\eta_{0}, \eta_{1}, \cdots, \eta_{n}\right)=0$ and $f(1,0, \cdots, 0) \neq 0$. If $\rho$ is the degree of $f$, the term $\eta_{0}^{\rho}$ must therefore occur in $f(\eta)$, and this proves our assertion.

(b) More generally, if the ideal $\left(\eta_{k+1}, \eta_{k+2}, \cdots, \eta_{n}\right)$ is irrelevant, then $\eta_{0}, \eta_{1}, \cdots, \eta_{k}$ are integrally dependent on $\eta_{k+1}, \eta_{k+2}, \cdots, \eta_{n}$. Proof by induction with respect to $k$ (that is, with respect to the number $k+1$ of elements $\eta_{i}$ which do not occur in the set $\eta_{k+1}, \eta_{k+2}, \cdots, \eta_{n}$ ). By (a), $\eta_{0}$ is integrally 
dependent on $\eta_{1}, \eta_{2}, \cdots, \eta_{n}$. Since every element of $0^{*}$ is integrally dependent on $\eta_{1}, \eta_{2}, \cdots, \eta_{n}$, the elements $\eta_{k+1}, \eta_{k+2}, \cdots, \eta_{n}$ generate in the ring $K\left[\eta_{1}, \eta_{2}, \cdots, \eta_{n}\right]$ an ideal of the same dimension as that of the ideal generated by them in $\mathrm{D}^{*}$, that is, they generate in $K\left[\eta_{1}, \eta_{2}, \cdots, \eta_{n}\right]$ an irrelevant ideal. By our induction it follows that $\eta_{1}, \eta_{2}, \cdots, \eta_{k}$ are integrally dependent on $\eta_{k+1}, \eta_{k+2}, \cdots, \eta_{n}$, q.e.d.

(c) If $\omega_{0}, \omega_{1}, \cdots, \omega_{r}$ are forms in $\eta_{0}, \eta_{1}, \cdots, \eta_{n}$, all of the same degree $h$, and if the ideal $\left(\omega_{0}, \omega_{1}, \cdots, \omega_{r}\right)$ is irrelevant, then the $\eta$ 's are integrally dependent on the $\omega$ 's. For if $\eta_{0}^{(h)}, \eta_{1}^{(h)}, \ldots$ form a linear base for the forms of degree $h$ in the $\eta$ 's and if we include the $\omega$ 's in this base, then applying (b) to the ring $K\left[\eta_{0}^{(h)}, \eta_{1}^{(h)}, \ldots\right]$, we conclude that $\eta_{0}^{h}, \eta_{1}^{h}, \cdots, \eta_{n}^{h}$ are integrally dependent on the $\omega$ 's.

(d) We obviously can select (in many ways) $r+1$ forms $\zeta_{0}, \zeta_{1}, \cdots, \zeta_{r}$ in o $^{*}$ such that the ideal $\left(\zeta_{0}, \zeta_{1}, \cdots, \zeta_{r}\right)$ be irrelevant. We can then find exponents $\sigma_{i}$ such that the forms $\omega_{i}=\zeta_{i}^{\sigma_{i}}$ be of like degree. Then it follows, by (c), that the $\eta$ 's are integrally dependent on $\omega_{0}, \omega_{1}, \cdots, \omega_{r}$. This completes the proof of the extended "normalization theorem" for homogeneous integral domains.

From an homogeneous integral domain $K\left[\eta_{0}, \eta_{1}, \cdots, \eta_{n}\right]$, of degree of transcendency $r+1$, we get an arbitrary integral domain $K\left[\xi_{1}, \xi_{2}, \cdots, \xi_{n}\right]$, of degree of transcendency $r$, by putting $\xi_{i}=\eta_{i} / \eta_{0}$. We apply step (d) above, and we observe that of the $r+1$ forms $\zeta_{i}$, one, say $\zeta_{0}$, can be taken arbitrarily. If we put $\zeta_{0}=\eta_{0}$, then $\omega_{0}$ is a power of $\eta_{0}$, say $\omega_{0}=\eta_{0}^{h}$. It is then seen immediately that the $r$ elements $\xi_{i}^{\prime}=\omega_{i} / \eta_{0}^{h}, i=1,2, \cdots, r$, are polynomials in the $\xi$ 's and that $\xi_{1}, \xi_{2}, \cdots, \xi_{n}$ are integrally dependent on $\xi_{1}^{\prime}, \xi_{2}^{\prime}, \cdots, \xi_{r}^{\prime}$. This completes the proof.

Let $\left(\eta_{0}, \eta_{1}, \cdots, \eta_{n}\right)$ be the general point of $V$ (the coordinates $\eta_{i}$ are homogeneous $\left({ }^{20}\right)$ and let $P=\mathrm{K}\left[\eta_{0}, \eta_{1}, \cdots, \eta_{n}\right]$. Let $\bar{P}$ be integral closure of $P$ in its quotient field. We first need to establish in the most general case that $\bar{P}$ is a finite $P$-module. By the normalization theorem of Emmy Noether, let $\zeta_{0}, \zeta_{1}, \cdots, \zeta_{r}$ be $r+1$ algebraically independent elements in $P$ such that every element of $P$ is integrally dependent on $\mathrm{K}\left[\zeta_{0}, \zeta_{1}, \cdots, \zeta_{r}\right]$. This last ring is a polynomial ring and we shall denote it by $R$. Since the field $\bar{\Sigma}=\mathbf{K}\left(\eta_{0}, \eta_{1}, \cdots, \eta_{n}\right)$ is a finite algebraic extension of the quotient field of the polynomial ring $R$, it follows that the integral closure $\bar{R}$ of $R$ in $\bar{\Sigma}$ is a finite $R$-module. This result, for arbitrary ground fields, has been proved by F. K. Schmidt [5]. Since $P \subseteq \bar{R}$, it follows that every element of $\bar{P}$ is integrally dependent on $\bar{R}$. But since $\bar{R}$ is a finite $R$-module and is therefore a chaintheorem ring, it is well known that every element of $\bar{\Sigma}$ which is integrally dependent on $\bar{R}$ is also integrally dependent on $R$, that is, belongs to $\bar{R}$. Hence $\bar{P} \subseteq \bar{R}$, that is, $\bar{P}=\bar{R}$. Thus $\bar{P}$ is a finite $R$-module, q.e.d.

(20) To avoid repetitions, we stipulate from now on that whenever the subscript in a set of coordinates begins with 0 , the coordinates are homogeneous. 
Since $\bar{P}$ is a finite $P$-module, it is a finite integral domain over $\mathrm{K}$. Let $\bar{P}=\mathrm{K}\left[\bar{\zeta}_{0}, \bar{\zeta}_{1}, \cdots, \bar{\zeta}_{m}\right]$. As in the quoted paper [7] (see p. 290), we may assume also here that the $\bar{\zeta}_{i}$ are homogeneous elements.

Then by exactly the same procedure as that carried out in our quoted paper we can show that if $\omega_{0}^{*}, \omega_{1}^{*}, \cdots, \omega_{\mu}^{*}$ is a linear K-basis for all the homogeneous elements of $\bar{P}$ of a given degree $\delta$, then for suitable integers $\delta$ it will be true that every homogeneous element in $\bar{P}$ whose degree is a multiple $\rho \delta$ of $\delta, \rho>0$, is necessarily a form of degree $\rho$ in $\omega_{0}^{*}, \omega_{1}^{*}, \cdots, \omega_{\mu}^{*}$. From that we concluded in the quoted paper that for such an integer $\delta$ the ring $P^{*}=\mathrm{K}\left[\omega_{0}^{*}, \omega_{1}^{*}, \cdots, \omega_{\mu}^{*}\right]$ is integrally closed in its quotient field, whence the variety $\bar{V}$ whose general point is $\left(\omega_{0}^{*}, \omega_{1}^{*}, \cdots, \omega_{\mu}^{*}\right)$ is normal. This variety $\bar{V}$ we termed a derived normal variety of $V$. It was pointed out to me by Irvin Cohen that the above conclusion fails to hold true if $\mathrm{K}$ is not maximally algebraic in the field $\Sigma$ of rational functions on $V$, that is, if $V$ is not absolutely irreducible (see [10, Lemma 4, p. 64]). For in this case the elements of $\Sigma$ which are algebraic over $\mathrm{K}$ but are not in $\mathrm{K}$, that is, the homogeneous elements of degree zero, are certainly not in the ring $P^{*}$. However, it is still true that $P^{*}$ contains all homogeneous integral quantities (that is, the homogeneous elements of the quotient field of $P^{*}$ which are integrally dependent of $P^{*}$ ) of positive degree. Hence if $\alpha^{*}$ is any integral quantity in the quotient field of $P^{*}$, then the products $\alpha^{*} \omega_{i}^{*}, i=0,1, \cdots, \mu$, belong to $P^{*}$, since they are sums of homogeneous integral quantities of positive degree. It follows that the irrelevant ideal $P^{*}\left(\omega_{0}^{*}, \omega_{1}^{*}, \cdots, \omega_{\mu}^{*}\right)$ is the conductor of the ring $P^{*}$ with respect to its integral closure in the quotient field of $P^{*}$. This implies that the variety $\bar{V}$ is locally normal in the sense of Definition 3 given later on in this section (see also [7, Theorem 13, p. 286]). For our purpose a locally normal variety is just as effective as a normal variety. We shall continue to call $\bar{V}$ the derived normal model of $V$, it being understood that if $V$ is not absolutely irreducible then $\bar{V}$ is only locally normal. It may be well to point out at this stage the self-evident fact that if $V$ is not absolutely irreducible, the field $\Sigma$ does not possess at all normal models over $\mathrm{K}$.

For the general theory of birational correspondences it is necessary to establish first some properties of the birational correspondence between a given model $V$ and a derived normal model $\bar{V}$ of $V$. For it will follow from the properties of this birational correspondence that the properties of a birational correspondence between any two models $V, V^{\prime}$ can be readily deduced from the properties of the birational correspondence between the derived normal models of $V$ and $V^{\prime}$. Therefore, there is no loss of generality if the theory is restricted to normal models. On the other hand, the emphasis on normal models is advantageous, both from a technical and a conceptual standpoint, since in the case of normal varieties the theory of birational correspondences is free from many accidental complications and irrelevant exceptions which one often encounters on non-normal varieties. 
Let us therefore consider the case in which one of the two birationally equivalent varieties $V, V^{\prime}$ is a derived normal model of the other. Let, say, $V^{\prime}$ be a derived normal model of $V$ and let $\left(\eta_{0}, \eta_{1}, \cdots, \eta_{n}\right)$ and $\left(\eta_{0}^{\prime}, \eta_{1}^{\prime}, \cdots, \eta_{m}^{\prime}\right)$ be the general points of $V$ and of $V^{\prime}$, respectively. Let $h$ be the degree of homogeneity of $V^{\prime}$. The elements $\eta_{i}^{\prime}$ form then a linear base for the elements of the field $\mathrm{K}\left(\eta_{0}, \eta_{1}, \cdots, \eta_{n}\right)$ which are integrally dependent on $\eta_{0}, \eta_{1}, \cdots, \eta_{n}$ and which are homogeneous of degree $h$. Moreover, the ring $P^{\prime}=\mathrm{K}\left[\eta_{0}^{\prime}, \eta_{1}^{\prime}, \cdots, \eta_{m}^{\prime}\right]$ is integrally closed (in its quotient field), or at any rate contains all homogeneous integers of positive degree.

It will be convenient to use an auxiliary projective model $V^{*}$ defined as follows. Let $\eta_{0}^{*}, \eta_{1}^{*}, \cdots, \eta_{s}^{*}$ be a linear base for the forms of degree $h$ in $\eta_{0}, \eta_{1}, \cdots, \eta_{n}$, with coefficients in $\mathrm{K}$. We take as $V^{*}$ the variety whose general point is $\left(\eta_{0}^{*}, \eta_{1}^{*}, \cdots, \eta_{s}^{*}\right)$.

LEMma 5. The birational correspondence between $V$ and $V^{*}$ is $(1,1)$ without exceptions $\left({ }^{21}\right)$. Any two corresponding irreducible subvarieties of $V$ and $V^{\prime}$ have the same dimension and the same quotient ring.

Proof. Let $W$ and $W^{*}$ be two corresponding irreducible subvarieties of $V$ and $V^{*}$, respectively. We assume that $\eta_{0} \neq 0$ on $W$. Since the $\eta^{*}$ 's constitute a linear base for the forms of degree $h$ in the $\eta$ 's, we may assume that $\eta_{0}^{h}$ is one of the $\eta^{*}$ 's, say $\eta_{0}^{h}=\eta_{0}^{*}$. If $v$ be a valuation of center $W$ and $W^{*}$, then $v\left(\eta_{i} / \eta_{0}\right) \geqq 0$, since $\eta_{0} \neq 0$ on $W$. From this it follows that $v\left(\eta_{j}^{*} / \eta_{0}^{*}\right) \geqq 0$, for $j=0,1, \cdots, s$, and hence (see I.3) $\eta_{0}^{*} \neq 0$ on $W^{*}$. Now let $\zeta$ be any element of $Q(W)$, say $\zeta=\phi(\eta) / \psi(\eta)$, where $\phi$ and $\psi$ are forms of like degree and where $\psi(\eta) \neq 0$ on $W$. We may assume that the common degree of $\phi$ and $\psi$ is a multiple of $h$, say $\rho h$, since we can multiply both $\phi$ and $\psi$ by any power of $\eta_{0}$ without destroying the inequality: $\psi \neq 0$ on $W$. But if $\phi$ and $\psi$ are of degree $\rho h$, then they can be expressed as forms of degree $\rho$ in the $\eta^{*}$ 's: $\phi(\eta)=\phi^{*}\left(\eta^{*}\right)$, $\psi(\eta)=\psi^{*}\left(\eta^{*}\right)$. Since $\psi(\eta) \neq 0$ on $W$, we have $v\left(\psi(\eta) / \eta_{0}^{\rho h}\right)=0$. Hence $v\left(\psi^{*}\left(\eta^{*}\right) / \eta_{0}^{* \rho}\right)=0$, and this shows that $\psi^{*}\left(\eta^{*}\right) \neq 0$ on $W^{*}$. Since $\zeta=\phi^{*}\left(\eta^{*}\right) / \psi^{*}\left(\eta^{*}\right)$, we conclude that $\zeta \in Q\left(W^{*}\right)$.

A quite similar argument shows that if $\zeta \in Q\left(W^{*}\right)$, then $\zeta \in Q(W)$. Hence the quotient rings $Q(W)$ and $Q\left(W^{*}\right)$ coincide, and from this our lemma follows in view of Theorem 3 (I.3).

The lemma shows that as far as the study of the birational correspondence between $V$ and $V^{\prime}$ is concerned, it is permissible to replace $V$ by $V^{*}$. Let us see therefore how $V^{\prime}$ is related to $V^{*}$.

Every homogeneous element in $\mathrm{K}\left(\eta_{0}, \eta_{1}, \cdots, \eta_{n}\right)$, of degree $h$,-and in particular each element $\eta_{i}^{\prime}$-can be written as a quotient of two forms in the $\eta$ 's whose degrees are multiples of $h$. Any such quotient is a quotient of

(21) When we say that a birational correspondence between two varieties $V$ and $V^{\prime}$ is $(1,1)$ without exceptions, we mean that it is $(1,1)$ as a correspondence between the irreducible subvarieties of $V$ and the irreducible subvarieties of $V^{\prime}$. 
two forms in the $\eta^{*}$ 's. Hence $\eta_{i}^{\prime} \in \mathrm{K}\left(\eta_{0}^{*}, \eta_{1}^{*}, \cdots, \eta_{s}^{*}\right)$. Conversely, each element $\eta_{i}^{*}$, being homogeneous of degree $h$, is a linear form in the $\eta^{\prime \prime}$ s. We therefore conclude that the two fields $\mathrm{K}\left(\eta_{0}{ }^{*}, \eta_{1}{ }^{*}, \cdots, \eta_{s}^{*}\right)$ and $\mathrm{K}\left(\eta_{0}^{\prime}, \eta_{1}^{\prime}, \cdots, \eta_{m}{ }^{\prime}\right)$ coincide.

The elements $\eta_{i}^{*}$, which as elements of the field $\mathrm{K}\left(\eta_{0}, \eta_{1}, \cdots, \eta_{n}\right)$ are homogeneous of degree $h$, as elements of the field $\mathrm{K}\left(\eta_{0}^{*}, \eta_{1}^{*}, \cdots, \eta_{s}^{*}\right)$ are to be regarded as homogeneous, of degree 1 . The same remark applies to the elements $\eta_{i}^{\prime}$. Moreover, the elements $\eta_{i}^{\prime}$ constitute a linear base for the elements of the field $\mathrm{K}\left(\eta_{0}{ }^{*}, \eta_{1}{ }^{*}, \cdots, \eta_{s}^{*}\right)$ which are homogeneous of degree 1 and which are integrally dependent on $\eta_{0}{ }^{*}, \eta_{1}^{*}, \cdots, \eta_{s}{ }^{*}$. We conclude from all this that $V^{\prime}$ is also a derived normal variety of $V^{*}$, of degree of homogeneity 1. Thus, while not every variety $V$ possesses a derived normal variety of degree of homogeneity 1 , we may nevertheless assume - and we do so assume-that we had originally $h=1$; this assumption amounts to replacing $V$ by $V^{*}$.

Now that we have $h=1$, it follows that $\eta$ 's are linear combinations of the $\eta^{\prime \prime}$ s, whence $V$ is a projection of the normal model $V^{\prime}$. Moreover, the ring $P^{\prime}=\mathrm{K}\left[\eta^{\prime}\right]$ is now either the integral closure of the ring $P=\mathrm{K}[\eta]$ in its quotient field or contains at any rate all homogeneous integral quantities of positive degree.

Let now $W$ and $W^{\prime}$ be corresponding irreducible subvarieties of $V$ and $V^{\prime}$, respectively. We assume that $\eta_{0} \neq 0$ on $W$ and that $\eta_{0}^{\prime}=\eta_{0} . I$ assert that $\eta_{0}^{\prime} \neq 0$ on $W^{\prime}$. To see this we have only to show that $v\left(\eta_{i}^{\prime} / \eta_{0}^{\prime}\right) \geqq 0$, $i=0,1, \cdots, m$, for at least one valuation of center $W^{\prime}$. We take as $v$ a valuation which has also $W$ as center on $V$. We write the relation of integral dependence for $\eta_{i}^{\prime}$ over $P$. It is of the form (see our paper $[7$, p. 286, equation (33)]):

$$
\eta_{i}^{\prime \nu}+a_{1}(\eta) \eta_{i}^{\prime \nu-1}+\cdots+a_{\nu}(\eta)=0,
$$

where $a_{j}(\eta)$ is a form of degree $j$ in $\eta_{0}, \eta_{1}, \cdots, \eta_{n}$. If we divide this equation by $\eta_{0}^{\nu}$, we see that the quotients $\eta_{i}^{\prime} / \eta_{0}^{\prime}$ are integrally dependent on the quotients $\eta_{1} / \eta_{0}, \eta_{2} / \eta_{0}, \cdots, \eta_{n} / \eta_{0}$. Since $\eta_{0} \neq 0$ on $W$, these quotients are in $R_{v}$, and this proves our assertion.

Let $\mathfrak{B}$ and $\mathfrak{P}^{\prime}$ be the prime homogeneous ideals of $W$ and of $W^{\prime}$ in the ring $P$ and $P^{\prime}$, respectively. From the fact that $\eta_{0} \neq 0$ on $W$ and $\eta_{0}^{\prime}\left(=\eta_{0}\right) \neq 0$ on $W^{\prime}$ and from the very definition of the center of a valuation, it follows immediately that $\mathfrak{B}=\mathfrak{B}^{\prime} \cap P$. Since the elements of $P^{\prime}$ are integrally dependent on $P$, there is only a finite number of prime ideals in $P^{\prime}$ which contract to $\mathfrak{B}$. These ideals are all homogeneous $\left({ }^{22}\right)$ and of the same dimension as $\mathfrak{B}$. We therefore reach the following conclusion:

To each irreducible subvariety $W^{\prime}$ of $V^{\prime}$ there corresponds a unique subvariety $W$ to $V$, while to each irreducible subvariety $W$ of $V$ there corresponds a finite

${ }^{22}$ ) The prime ideals $\mathfrak{P}^{\prime}$ which contract to $\mathfrak{P}$ are the minimal primes of the extended ideal $P^{\prime} \cdot \mathfrak{B}$ and therefore are homogeneous, by Theorem 2 (I.1). 
number of subvarieties $W^{\prime}$ of $V^{\prime}$. Two corresponding varieties $W$ and $W^{\prime}$ have the same dimension.

We now investigate the relationship between the quotient rings of two corresponding varieties $W$ and $W^{\prime}$. We pass to the rings $\mathfrak{D}=\mathbf{K}\left[\xi_{1}, \xi_{2}, \cdots, \xi_{n}\right]$ and $\mathfrak{D}^{\prime}=\mathrm{K}\left[\xi_{1}^{\prime}, \xi_{2}^{\prime}, \cdots, \xi_{m}^{\prime}\right]$ of the nonhomogeneous coordinates $\xi_{i}=\eta_{i} / \eta_{0}$ and $\xi_{i}^{\prime}=\eta_{i}^{\prime} / \eta_{0}^{\prime}$, where $\eta_{0}=\eta_{0}^{\prime}$. Here $\mathfrak{o}^{\prime}$ is the integral closure of $\mathfrak{o}$. Let $\mathfrak{p}$ be the prime $\mathrm{D}$-ideal of $W$. Any $W^{\prime}$ which corresponds to $W$ will be at finite distance with respect to the coordinates $\xi_{i}^{\prime}$ and will be given in $\mathfrak{v}^{\prime}$ by a prime ideal which contracts to $\mathfrak{p}$. Let $\mathfrak{p}_{1}^{\prime}, \mathfrak{p}_{2}^{\prime}, \cdots, \mathfrak{p}_{\nu}^{\prime}$ be the prime $\mathfrak{o}^{\prime}$-ideals which contract to $\mathfrak{p}$, and let $W_{1}^{\prime}, W_{2}^{\prime}, \cdots, W_{\nu}^{\prime}$ be the corresponding subvarieties of $V^{\prime}$. Let

$$
\Im=Q(W)=\mathfrak{o p}_{\mathfrak{p}}, \quad \Im_{i}^{\prime}=Q\left(W_{i}^{\prime}\right)=\mathrm{op}_{\mathfrak{i}}^{\prime},
$$

and let $\mathfrak{m}$ and $\mathfrak{m}_{i}^{\prime}$ denote the ideals of non-units in $\Im$ and $\Im_{i}^{\prime}$, respectively. We have $\Im_{i}^{\prime} \supseteq \Im$ and $\Im_{i}^{\prime}$ is integrally closed. Let $\Im^{*}$ denote the integral

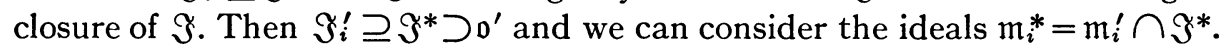
The ideals $\mathrm{m}_{1}^{*}, \mathfrak{m}_{2}^{*}, \cdots, \mathfrak{m}_{\nu}^{*}$ are distinct, since $\mathfrak{m}_{i}^{*} \cap \mathfrak{o}^{\prime}=\mathfrak{m}_{i}^{\prime} \cap \mathfrak{o}^{\prime}=\mathfrak{p}_{i}^{\prime}$. The ideals $\mathfrak{m}_{i}^{*}$ contract to one and the same ideal in $\Im$, namely to $\mathfrak{m}$, since $\mathfrak{p}_{i}^{\prime} \cap \mathfrak{D}=\mathfrak{p}$ and $\mathfrak{m}=\mathfrak{\Im} \cdot \mathfrak{p}$. The quotient ring of $\mathrm{m}_{i}^{*}$ in $\mathfrak{S}^{*}$ is contained in $\mathfrak{\Im}_{i}^{\prime}$ since $\mathfrak{m}_{i}^{*}=\mathfrak{m}_{i}^{\prime} \cap \mathfrak{J}^{*}$. On the other hand, the quotient ring $\Im_{i}^{\prime}$ is contained in the quotient ring of $\mathrm{m}_{i}^{*}$, since $\mathrm{m}_{i}^{*} \cap \mathfrak{o}^{\prime}=\mathfrak{p}_{i}^{\prime}$. Hence the quotient ring of $\mathrm{m}_{i}^{*}$ in the ring $\Im^{*}$ coincides with $\Im_{i}^{\prime}$.

The foregoing properties of $\Im^{*}$ can also be derived from the general theory of quotient rings (see I.2 $\left(^{1}\right)$ ). It is only necessary to observe that $\Im^{*}$ coincides with the quotient ring $\mathfrak{o}_{S}^{\prime}$, where $S=\mathfrak{p}-\mathfrak{p}$. From this remark it follows immediately that the ideals $\mathfrak{m}_{1}^{*}, \mathfrak{m}_{2}{ }^{*}, \cdots, \mathfrak{m}_{\nu}{ }^{*}$ are the only prime ideals of $\Im^{*}$ which contract to $\mathrm{m}$. The connection between the quotient ring $Q(W)$ and the $\nu$ quotient rings $Q\left(W_{i}^{\prime}\right)$ is therefore fully established. Reassuming, we can now state the following theorem:

THEOREM 7. The birational correspondence between an irreducible algebraic variety $V$ and a derived normal variety $V^{\prime}$ of $V$ has the following properties:

(A) Two corresponding subvarieties $W$ and $W^{\prime}$ of $V$ and $V^{\prime}$, respectively, have the same dimension, and we have: $Q(W) \subseteq Q\left(W^{\prime}\right)$.

(B) Given $W^{\prime}$, the corresponding $W$ is uniquely determined, while to a given $W$ there corresponds a finite number of varieties $W^{\prime}$.

(C) If $\Im^{*}$ denotes the integral closure of the quotient ring $\Im=Q(W)$, and if $\mathrm{m}_{1}^{*}, \mathrm{~m}_{2}^{*}, \cdots, \mathrm{m}^{*}$ are the prime ideals in $\Im^{*}$ which contract to the ideal of non-units in $\Im$, then there are exactly $\nu$ varieties $W_{1}^{\prime}, W_{2}^{\prime}, \cdots, W_{\nu}^{\prime}$ which correspond to $W$, and for a suitable ordering of the indices we will have $Q\left(W_{i}^{\prime}\right)=\Im_{\mathrm{m}_{i}^{*}}^{*}$.

COROLlARY 1. The birational correspondence between any two derived normal varieties of $V$ is $(1,1)$ without exceptions, and corresponding subvarieties have the same quotient ring. 
Other corollaries follow from Theorem 7 . We first give the following definitions:

Definition 2. If $Q(W)$ is integrally closed, then $V$ is said to be locally normal at $W$.

DEFINITION 3. If $V$ is locally normal at each of its irreducible subvarieties, then $V$ is said to be a locally normal variety $\left({ }^{23}\right)$.

A locally normal variety is characterized by the property that it is normal in the affine space for every choice of the nonhomogeneous coordinates. For that it is necessary and sufficient (see our paper [7, Theorem 13, p. 286]) that the conductor of the ring $P=\mathrm{K}\left[\eta_{0} ; \eta_{1}, \cdots, \eta_{n}\right]$ with respect to the integral closure of $P$ be an irrelevant ideal.

Corollary 2. If $V$ is locally normal at $W$ then to $W$ there corresponds a unique subvariety $W^{\prime}$ of the derived normal model $V^{\prime}$, and the quotient rings $Q(W), Q\left(W^{\prime}\right)$ coincide.

CoRollary 3. If $V$ is locally normal, then the birational correspondence between $V$ and a derived normal variety $V^{\prime}$ of $V$ is $(1,1)$ without exceptions, and the correspondence preserves quotient rings.

COROLlary 4. The irreducible subvarieties of $V$ to which there corresponds more than one variety on $V^{\prime}$ all lie on the subvariety $C$ of $V$ which is defined by the conductor 5 of the ring $P=\mathrm{K}\left[\eta_{0}, \eta_{1}, \cdots, \eta_{n}\right]$ with respect to the integral closure of $P$. Outside of $C$, the birational correspondence between $V$ and $V^{\prime}$ is $(1,1)$ and it preserves quotient rings.

3. The fundamental elements of a birational correspondence. We consider a birational correspondence $T$ between two locally normal varieties $V$ and $V^{\prime}$ (the general case is discussed briefly at the end of this section). Let $W$ be an irreducible subvariety of $V$.

Definition 4. We say that $W$ is (1) regular, (2) irregular, or (3) fundamental for $T$ if there exists a $W^{\prime}$ on $V^{\prime}$ such that $W^{\prime}=T(W)$ and, respectively, (1) $Q(W)=Q\left(W^{\prime}\right)$, (2) $Q(W) \supset Q\left(W^{\prime}\right)$ or (3) $Q(W) \nsupseteq Q\left(W^{\prime}\right)$.

The following theorem is merely a statement of some properties of regular, irregular and fundamental varieties which follow directly from the definition and from Theorem 6 (II.1) and which we shall use very frequently:

THEOREM 8.

(A) If $W$ is regular or irregular, then to $W$ there corresponds a unique $W^{\prime}$ on $V^{\prime}$.

${ }^{(23)}$ For $V$ to be locally normal it is sufficient that $V$ be locally normal at each of its points. For if $W$ is any irreducible subvariety of $V$ and if $P$ is any point of $W$, then $Q(W)$ is also the quotient ring of a prime ideal in $Q(P)$. If $Q(P)$ is integrally closed, also $Q(W)$ is integrally closed. 
(B) If $W$ is regular for $T$ and if $W^{\prime}=T(W)$, then $W^{\prime}$ is of the same dimension as $W$ and is regular for $T^{-1}$.

(C) If $W$ is irregular and $W^{\prime}=T(W)$, then $W^{\prime}$ is fundamental for $T^{-1}$, and the dimension of $W^{\prime}$ is less than or equal to the dimension of $W$.

(D) If $W$ is fundamental, then $Q(W) \nsupseteq Q\left(W^{\prime}\right)$ for any $W^{\prime}$ which corresponds to $W$.

(E) $A$ necessary and sufficient condition that $W$ not be fundamental is that for a suitable choice of the nonhomogeneous coordinates $\xi_{1}^{\prime}, \xi_{2}^{\prime}, \cdots, \xi_{m}^{\prime}$ of the general point of $V^{\prime}$, the ring $\mathrm{D}^{\prime}$ of these coordinates be contained in $Q(W)$.

The proof of $(\mathrm{E})$ is immediate. For assume that $\mathfrak{v}^{\prime} \subseteq Q(W)$ and let $\mathfrak{p}^{\prime}$ be the prime $\mathfrak{o}^{\prime}$-ideal which is the contraction of the ideal of non-units of $Q(W)$. Then if $W^{\prime}$ is the irreducible subvariety of $V^{\prime}$ which is defined by $\mathfrak{p}^{\prime}$, then $Q\left(W^{\prime}\right) \subseteq Q(W)$ and by Theorem 3 every valuation of center $W$ on $V$ has center $W^{\prime}$ on $V^{\prime}$.

The sets of regular, irregular and fundamental varieties are mutually exclusive. We now give the following definition:

Definition 5. A birational correspondence $T$ is regular, if every $W$ is regular for $T$.

It is clear that if $T$ is regular, then also $T^{-1}$ is regular (Theorem 8 (B)) A regular birational correspondence is a $(1,1)$ correspondence, without exceptions, and it preserves quotient rings. We have encountered examples of regular birational correspondences in the preceding section (Lemma 5; Theorem 7, Corollaries 1 and 3).

The next theorem shows that Theorem 8 (A) expresses a characteristic property of a non-fundamental $W$.

THEOREM 9. If to $W$ there corresponds a unique subvariety $W^{\prime}$ of $V^{\prime}$, then $W$ is not fundamental for $T$ (hence is either regular or irregular).

Proof. Since $V$ is locally normal, the quotient ring $Q(W)$ is integrally closed. By the fundamental theorem on principal orders $\left({ }^{14}\right), Q(W)$ is the intersection of the valuation rings which contain $Q(W)$. Let $R_{v_{1}}$ be one of these valuation rings. Since $R_{v_{1}} \supseteq Q(W)$, it follows that the center of $v_{1}$ on $V$ is either $W$ or a subvariety $W_{1}$ of $V$ which properly contains $W$ (Theorem 3 , I.3). In the second case there exists a valuation $v$ which is compounded with $v_{1}$ and has center $W$ (Lemma 4, I.4). Since $R_{v} \subset R_{v_{1}}$ we can omit $R_{v_{1}}$ from the set of valuation rings which contain $Q(W)$, without affecting the intersection of these rings. Hence $Q(W)$ is also the intersection of the valuation rings which belong to valuations of center $W$. Since, by hypothesis, all valuations of center $W$ on $V$ have the same center $W^{\prime}$ on $V^{\prime}$, it follows that the corresponding valuation rings all contain $Q\left(W^{\prime}\right)$. Consequently $Q(W) \supseteq Q\left(W^{\prime}\right)$, as was asserted. 
Corollary. The dimension of a fundamental variety $W$ cannot exceed $r-2$.

For if $W$ has dimension $r-1$, then $Q(W)$ is itself a valuation ring, namely the valuation ring of a divisor $v$. Therefore $W$ is the center of only one valuation, namely of the divisor $v$.

THEOREM 10. If $W$ is fundamental, then to $W$ there correspond on $V^{\prime}$ infinitely many varieties $W^{\prime}$.

Proof. We shall prove that if to $W$ there corresponds on $V^{\prime}$ only a finite number of varieties, then $W$ is not fundamental.

Let $W_{1}^{\prime}, W_{2}^{\prime}, \cdots, W_{h}^{\prime}$ be the irreducible subvarieties of $V^{\prime}$ which correspond to $W$. If $v$ is any valuation of center $W$, then $R_{v}$ must contain at least one of the $h$ quotient rings $Q\left(W_{i}^{\prime}\right)$. Hence $R_{v}$ contains the intersection of these quotient rings. Since $Q(W)$ is the intersection of all $R_{v}$, it follows that $Q(W)$ contains the intersection of the $h$ quotient rings $Q\left(W_{i}^{\prime}\right)$.

We can find a form $\phi\left(\eta_{0}^{\prime}, \eta_{1}^{\prime}, \cdots, \eta_{m}^{\prime}\right)$, of a sufficiently high degree $\nu$, such that $\phi \neq 0$ on $W_{i}^{\prime}, i=1,2, \cdots, h$. We pass from $V^{\prime}$ to the variety $V_{1}^{\prime}$ whose general point is defined by a linear K-basis of the forms of degree $\nu$ in $\eta_{0}^{\prime}, \eta_{1}^{\prime}, \cdots, \eta_{m}^{\prime}$. By Lemma $5, V^{\prime}$ and $V_{1}^{\prime}$ are in regular birational correspondence, hence we may replace in our proof $V^{\prime}$ by $V_{1}^{\prime}$. We may therefore assume that $\phi$ is one of the elements $\eta_{i}^{\prime}$, say $\phi=\eta_{0}^{\prime}$. From the fact that $\eta_{0}^{\prime} \neq 0$ on $W_{i}^{\prime}, i=1,2, \cdots, h$, it follows that the ring $\mathfrak{o}^{\prime}$ of the nonhomogeneous coordinates $\xi_{i}^{\prime}=\eta_{i}^{\prime} / \eta_{0}^{\prime}$ is contained in each quotient ring $Q\left(W_{i}^{\prime}\right)$. Since the intersection of the rings $Q\left(W_{i}^{\prime}\right)$ is contained in $Q(W)$, our theorem follows from Theorem 8 (E).

We shall now discuss briefly the general case in which $V$ and $V^{\prime}$ are not locally normal. Let $\bar{V}$ and $\bar{V}^{\prime}$ be derived normal varieties of $V$ and $V^{\prime}$ respectively. Let $W$ be an irreducible subvariety of $V$ and let $\bar{W}_{1}, \bar{W}_{2}, \cdots, \bar{W}_{h}$ be the irreducible varieties on $\bar{V}$ which correspond to $W$ (Theorem 7 (B)). We shall denote by $\bar{T}$ the birational correspondence between $\bar{V}$ and $\bar{V}^{\prime}$.

Definition 6. The variety $W$ is regular for $T$, if each $\bar{W}_{i}, i=1,2, \cdots, h$, is regular for $\bar{T} ; W$ is fundamental for $T$, if at least one of the varieties $\bar{W}_{i}$ is fundamental for $\bar{T} ; W$ is irregular for $T$, if it is neither regular nor fundamental, that is, if no $\bar{W}_{i}$ is fundamental for $\bar{T}$ and if at least one $\bar{W}_{i}$ is irregular for $\bar{T}$.

Of the theorems proved in this section for locally normal varieties, Theorems 9 and 10 continue to hold in the general case. The validity of Theorem 10 is obvious. As to Theorem 9, the proof is as follows. If $T$ is single-valued at $W$, say $T(W)=W^{\prime}$, then any irreducible subvariety of $\bar{V}^{\prime}$ which corresponds to $\bar{W}_{i}$ under $\bar{T}(i=1,2, \cdots, h)$ must be among the irreducible subvarieties of $\bar{V}^{\prime}$ which correspond to $W^{\prime}$ in the birational correspondence between $V^{\prime}$ and $\bar{V}^{\prime}$. Hence to each $\bar{W}_{i}$ there can correspond on $\bar{V}^{\prime}$ only a finite number of varieties. Therefore no $\bar{W}_{i}$ is fundamental (Theorem 10) for $\bar{T}$, and therefore, by definition, $W$ is not fundamental for $T$. 
In particular, if $T(W)=W^{\prime}$ and if $Q(W) \supseteq Q\left(W^{\prime}\right)$, then $W$ is not fundamental. This follows from Theorem 6, II.1.

On the other hand, other results established for locally normal varieties do not generalize to varieties which are not locally normal. For instance, parts (A) and (B) of Theorem 8 cease to be true in the general case. Also the defining property of a non-fundamental variety used in Definition 4 ceases to be a property of non-fundamental varieties in the general case, that is, if $W$ is not fundamental that does not mean that there must exist a $W^{\prime}$ such that $W^{\prime}=T(W)$ and $Q(W) \supseteq Q\left(W^{\prime}\right)$. Also the condition stated in Theorem $8(\mathrm{E})$ is sufficient, but no longer necessary.

Note that according to Definition 6 the birational correspondence between a variety $V$ and derived normal variety of $V$ is free from fundamental elements on either variety.

We shall agree to use Definition 5 of regular birational correspondences also in the case of varieties which are not locally normal.

4. A question of terminology. At this stage it becomes necessary to point out and to discuss the difference between our terminology and the terminology used heretofore in the literature. This difference concerns the meaning of the term "fundamental" and our use of the new term "irregular."

In the case of algebraic surfaces it is the sense of the old terminology that both points and curves can be fundamental: a point $P$ is fundamental if it is transformed into a curve $\Gamma^{\prime}$, and any such curve $\Gamma^{\prime}$, which is then the transform of a point, is "fundamental." As far as the notion of a fundamental point is concerned this is in agreement with our terminology, from Theorem 8 (A) and Theorem 10. However, by Theorem 9, corollary, a curve on an algebraic surface can never be fundamental in our sense. The "fundamental" curves in the sense of the old terminology are irregular curves in our sense.

The reasons for our terminology-or better-the inadequacy of the old terminology $\left({ }^{24}\right)$ become apparent in the case of higher varieties. Let us consider, for instance, a birational correspondence $T$ between two 3-dimensional varieties $V$ and $V^{\prime}$. Again, according to the old terminology we may have "fundamental" loci of all dimensions from 0 to 2 . As far as fundamental points and "fundamental" surfaces are concerned, the situation is the same as in the case of algebraic surfaces: there is complete agreement on fundamental points, while according to our terminology there are definitely no "fundamental" surfaces, but only irregular surfaces. It is, however, the use of the term "fundamental curve" that brings out some significant facts.

I can find no clear-cut definition of a fundamental curve in the literature. This much is certain: if a curve $\Gamma$ is such that $T(\Gamma)$ is a surface, or if $\Gamma$ corresponds to each point of another curve, then in the old terminology (and

(24) The best justification for our terminology is its own logical consistency. We call fundamental a variety $W$ if and only if the birational correspondence $T$ is infinitely many-valued at $W$. Otherwise $W$ is either regular or irregular. 
also in our terminology) $\Gamma$ is fundamental (respectively, of the "first" or of the "second kind"). Suppose, however, that the transform of $\Gamma$ is a single point. I am not certain whether or not such a curve is fundamental in the sense of the old terminology. If it is, then the terminology is confusing, since we are dealing here with a curve at which the birational transformation is single-valued. If it is not, then the terminology is inconsistent, in view of the use of the term "fundamental" surface, since in both cases we are dealing with a $W$ such that $T(W)$ is unique and is of lower dimension than $W$.

It is quite possible that in the old terminology no special name has ever been assigned to a curve $\Gamma$ such that $T(\Gamma)$ is a single point. If that is the case, then this is probably due to the fact that, as a rule, only nonsingular models have been considered in the literature. If the three-dimensional varieties $V$ and $V^{\prime}$ are nonsingular, then a curve $\Gamma$ which is transformed into a point necessarily lies on a surface which is transformed into a curve (see II.10, Theorem 17, corollary). Thus, such a curve $\Gamma$ always lies on a "fundamental" surface, and there seemed to be no compelling reason for giving these curves a special name. However, in the case of singular models it may very well happen that a curve $\Gamma$ whose dimension is lowered by the birational transformation $T$ and at which $T$ is single-valued (these two properties imply that $W$ is irregular; see Theorem 8 (B) and Theorem 9) does not lie on any surface having the same properties (compare with Theorem 17, II.10). Some term for such a curve is necessary, and the term "fundamental" we reject for reasons given above.

5. The join of two birationally equivalent varieties. Let $\left(\eta_{0}, \eta_{1}, \cdots, \eta_{n}\right)$ and $\left(\eta_{0}^{\prime}, \eta_{1}^{\prime}, \cdots, \eta_{m}^{\prime}\right)$ be the general points, respectively, of $V$ and of $V^{\prime}$, where $V$ and $V^{\prime}$ are our two birationally equivalent varieties. Since the quotients $\eta_{i}^{\prime} / \eta_{0}^{\prime}$ are rational functions of the quotients $\eta_{j} / \eta_{0}$, the $\eta^{\prime \prime}$ 's are proportional to forms of like degree in the $\eta$ 's:

$$
\begin{aligned}
\eta_{0}^{\prime}: \eta_{1}^{\prime}: \cdots: \eta_{m}^{\prime} \\
\quad=\phi_{0}\left(\eta_{0}, \eta_{1}, \cdots, \eta_{n}\right): \phi_{1}\left(\eta_{0}, \eta_{1}, \cdots, \eta_{n}\right): \cdots: \phi_{m}\left(\eta_{0}, \eta_{1}, \cdots, \eta_{n}\right) .
\end{aligned}
$$

Definition 7. The irreducible algebraic variety $V^{*}$ whose general point has the $(n+1)(m+1)$ products $\eta_{i} \phi_{j}$ as homogeneous coordinates is called the join of $V$ and $V^{\prime}$.

We shall denote the products $\eta_{i} \phi_{j}$ by $\eta_{i j}$ and the quotients $\eta_{i} / \eta_{0}, \eta_{j}^{\prime} / \eta_{0}^{\prime}$ and $\eta_{i j} / \eta_{00}$ by $\xi_{i}, \xi_{j}^{\prime}$ and $\xi_{i j}$, respectively. We have then:

$$
\xi_{i 0}=\xi_{i}, \quad \xi_{0 j}=\xi_{j}^{\prime}, \quad \xi_{i j}=\xi_{i} \xi_{j}^{\prime}, \quad i, j \neq 0,
$$

and from these relations it follows that $V^{*}$ is birationally equivalent to $V$ (and to $V^{\prime}$ ). Moreover, if we take as nonhomogeneous coordinates of the general point of $V^{*}$ the quotients of the $\eta_{i j}$ 's by a fixed $\eta_{i j}$, say by $\eta_{00}$, then the ring of these coordinates is, by (5), the join of the two rings of nonhomogene- 
ous coordinates relative to $V$ and $V^{\prime}$. In symbols: if

$$
\begin{gathered}
\mathfrak{D}=\mathrm{K}\left[\xi_{1}, \xi_{2}, \cdots, \xi_{n}\right], \quad \mathfrak{o}^{\prime}=\mathrm{K}\left[\xi_{1}^{\prime}, \xi_{2}^{\prime}, \cdots, \xi_{m}^{\prime}\right], \\
\mathfrak{o}^{*}=\mathrm{K}\left[\xi_{10}, \xi_{20}, \cdots, \xi_{n m}\right],
\end{gathered}
$$

then $\mathfrak{D}^{*}=\left(\mathfrak{D}, \mathfrak{D}^{\prime}\right)$.

THEOREM 11. In the birational correspondence $T^{*}$ between $V$ and $V^{*}$ there corresponds to any irreducible subvariety $W^{*}$ of $V^{*}$ a unique subvariety $W$ of $V$. If a given $W$ on $V$ is not fundamental for $T$, then it is regular for $T^{*}$. Similarly for $V^{\prime}$ and $V^{*}$.

Proof. We may assume that $W^{*}$ is at finite distance with respect to the nonhomogeneous coordinates $\xi_{i j}$. If $v$ is any valuation of center $W^{*}$, then $R_{v} \supseteq Q\left(W^{*}\right) \supset \mathfrak{o}^{*} \supseteq \mathfrak{0}$, whence the center $W$ of $v$ on $V$ is at finite distance with respect to the nonhomogeneous coordinates $\xi_{i}$. Similarly for $V^{\prime}, W^{\prime}$ and the $\xi_{j}^{\prime}$. But then, if $\mathfrak{p}^{*}$ is the prime $\mathfrak{o}^{*}$-ideal of $W^{*}$, the prime ideals $\mathfrak{p}$ and $\mathfrak{p}^{\prime}$ of $W$ and $W^{\prime}$, in the rings $\mathfrak{D}$ and $\mathfrak{o}^{*}$, respectively, are necessarily the contracted ideals of $\mathfrak{p}^{*}$, that is, $\mathfrak{p}=\mathfrak{p}^{*} \cap \mathfrak{o}, \mathfrak{p}^{\prime}=\mathfrak{p}^{*} \cap \mathfrak{o}^{\prime}$, and consequently $W$ and $W^{\prime}$ are uniquely determined by $W^{*}$. Notice that the quotient rings $Q(W)$ and $Q\left(W^{\prime}\right)$ are subrings of $Q\left(W^{*}\right)$ :

$$
Q(W) \subseteq Q\left(W^{*}\right), \quad Q\left(W^{\prime}\right) \subseteq Q\left(W^{*}\right) .
$$

To prove the second part of the theorem, let $T(W)=W^{\prime}$ and let us assume that $W$ and $W^{\prime}$ are at finite distance with respect to the nonhomogeneous coordinates $\xi_{i}$ and $\xi_{j}^{\prime}$. Let $v$ be any valuation of center $W$ and $W^{\prime}$ on $V$ and $V^{\prime}$, respectively. The center $W^{*}$ of $v$ on $V^{*}$ will be at finite distance with respect to the coordinates $\xi_{i j}$, since $R_{v} \supset \mathfrak{v}, R_{v} \supset \mathrm{o}^{\prime}$ and therefore $R_{v} \supset \mathrm{o}^{*}$.

Let us first consider the case in which $V$ is locally normal. Since $W$ is not fundamental for $T$, we have $Q(W) \supseteq Q\left(W^{\prime}\right) \supset \mathfrak{o}^{\prime}$. Therefore $Q(W) \supset \mathfrak{o}^{*}$, that is, $\mathfrak{p}_{\mathfrak{p}} \supset \mathfrak{p}^{*}$. Since $\mathfrak{p}^{*} \cap \mathfrak{p}=\mathfrak{p}$, it follows that $\mathfrak{p}_{\mathfrak{p}}$ contains the ring $\mathfrak{o}_{\mathfrak{p}^{*}}^{*}$, that is, $Q(W) \supseteq Q\left(W^{*}\right)$. Hence, by (6), $Q(W)=Q\left(W^{*}\right)$, whence $W$ is regular for $T^{*}$.

To prove the theorem in the general case we first observe that if $\bar{V}$ and $\bar{V}^{\prime}$ denote derived normal varieties of $V$ and of $V^{\prime}$, respectively, then any derived normal variety $\bar{V}^{*}$ of the join of $V$ and $V^{\prime}$ is in regular birational correspondence with any derived normal variety of the join of $\bar{V}$ and $\bar{V}^{\prime}$. The proof is straightforward and consists in the obvious remark that the integral closure of the ring $\left(\mathfrak{D}, \mathfrak{D}^{\prime}\right)$ is the same as integral closure of the ring $\left(\mathfrak{o}_{1}, \mathfrak{D}_{1}^{\prime}\right)$, where $\mathfrak{D}_{1}$ and $\boldsymbol{o}_{1}^{\prime}$ are the integral closures of $\mathfrak{D}$ and of $\mathfrak{D}^{\prime}$, respectively. Now let $\bar{W}$ be any of the irreducible subvarieties of $\bar{V}$ which correspond to $W$. To prove that $W$ is regular for $T^{*}$ we have only to prove (Definition 6, II.3) that $\bar{W}$ is regular for the birational correspondence between $\bar{V}$ and $\bar{V}^{*}$. Since $W$, by hypothesis, is not fundamental for $T$, it follows (Definition 6) that $\bar{W}$ is not fundamental for the birational correspondence between $\bar{V}$ and $\bar{V}^{\prime}$. Since $\bar{V}$ is locally normal, it follows by the case just considered that $\bar{W}$ is regular 
for the birational correspondence between $\bar{V}$ and the join of $\bar{V}$ and $\bar{V}^{\prime}$. Since a derived normal variety of this join is, by the remark made above, in regular birational correspondence with $\bar{V}^{*}$, it follows that $\bar{W}$ is regular for the birational correspondence between $\bar{V}$ and $\bar{V}^{*}$, as was asserted.

Corollary. If $P$ and $P^{\prime}$ are corresponding points of $V$ and $V^{\prime}$, then there is only a finite number of points $P^{*}$ on $V^{*}$ which correspond to both $P$ and $P^{\prime}$. If $V$ (or $\left.V^{\prime}\right)$ is locally normal, then the number of such points $P^{*}$ can be greater than 1 only if $P$ (or $P^{\prime}$ ) is a fundamental point of $T$ (or of $T^{-1}$ ).

For if we identify the varieties $W, W^{\prime}$ and $W^{*}$ of the preceding proof with the points $P, P^{\prime}$ and $P^{*}$, respectively, we see that $p^{*}$ must be a zerodimensional prime divisor of the ideal $\mathfrak{p}^{*} \cdot\left(\mathfrak{p}, \mathfrak{p}^{\prime}\right)$. Since this ideal is pure zerodimensional, the number of possible ideals $p^{*}$ is finite. The second half of the corollary follows directly from the second half of the preceding theorem.

If the ground field $K$ is algebraically closed, the ideal $\mathfrak{o}^{*} \cdot\left(\mathfrak{p}, \mathfrak{p}^{\prime}\right)$ is itself prime, whenever $\mathfrak{p}$ and $\mathfrak{p}^{\prime}$ are both zero-dimensional. Hence if $K$ is algebraically closed, then not only does every point $P^{*}$ of $V^{*}$ determine uniquely a pair of corresponding points $P, P^{\prime}$ of $V$ and $V^{\prime}$, respectively, but, conversely, every such pair determines uniquely a point $P^{*}$ on $V^{*}$. For this reason the join $V^{*}$ is often referred to in the literature as the variety of pairs of corresponding points of $V$ and $V^{\prime}$.

If $\mathrm{K}$ is not algebraically closed then $P^{*}$ need not be uniquely determined by $P$ and $P^{\prime}$. The following is an example $\left({ }^{25}\right)$. Let $\mathrm{K}$ be the field of real numbers and let $\Sigma=\mathrm{K}(x, y)$, where $x$ and $y$ are indeterminates. We take as $V$ and $V^{\prime}$ two planes given-in nonhomogeneous coordinates-by the general points $\left(x, y_{1}\right)$ and $\left(x_{1}, y\right)$, respectively, where $y_{1}=y\left(x^{2}+1\right)$ and $x_{1}=x\left(y^{2}+1\right)$. Here we have: $\mathfrak{D}=\mathrm{K}\left[x, y_{1}\right], \quad \mathfrak{o}^{\prime}=\mathrm{K}\left[x_{1}, y\right], \mathfrak{o}^{*}=\left(\mathfrak{\mathfrak { o }}, \mathfrak{o}^{\prime}\right)=\mathrm{K}\left[\begin{array}{ll}x, y\end{array}\right]$. Let $\mathfrak{p}=\left(x^{2}+1, y_{1}\right), \mathfrak{p}^{\prime}=\left(x_{1}, y^{2}+1\right)$. These ideals are prime and zero-dimensional in their respective rings and they represent corresponding points of the two planes $V$ and $V^{\prime}$. However, the ideal $\mathfrak{p}^{*} \cdot\left(\mathfrak{p}, \mathfrak{p}^{\prime}\right)$ is now the intersection of the following prime ideals: $\mathfrak{p}_{1}^{*}=\left(x^{2}+1, y-x\right), \mathfrak{p}_{2}^{*}=\left(x^{2}+1, y+x\right)$.

The join $V^{*}$ of two locally normal varieties $V$ and $V^{\prime}$ need not be locally normal. One may often find it convenient to pass from $V^{*}$ to a derived normal variety $\bar{V}^{*}$ of $V^{*}$. We may call $\bar{V}^{*}$ the normal join of $V$ and $V^{\prime}$.

(25) It should not be too difficult to find necessary and sufficient conditions in order that a given pair of corresponding points $P, P^{\prime}$ determine uniquely a point $P^{*}$ of the join $V^{*}$. The following is a sufficient condition. If $\Delta=\mathfrak{0} / \mathfrak{p}$ and $\Delta^{\prime}=\mathfrak{0}^{\prime} / \mathfrak{p}^{\prime}$ are the residue fields of the points $P$ and $P^{\prime}$ respectively, then a least field $\Delta^{*} / \mathrm{K}$ containing $\Delta / \mathrm{K}$ and $\Delta^{\prime} / \mathrm{K}$ should exist such that its relative degree over $\mathrm{K}$ is the product of the relative degrees of $\Delta$ and of $\Delta^{\prime}$ over $\mathrm{K}$. (It is not difficult to see that this product is the maximum value for the relative degree of $\Delta^{*}$ over $\mathrm{K}$, and if that maximum is reached, then there exists, to within relative isomorphisms, only one least field which contains $\Delta$ and $\Delta^{\prime}$.) However, the above condition is not sufficient. In fact, no condition can be both necessary and sufficient which does not take into account the quotient rings $Q(P)$ and $Q\left(P^{\prime}\right)$ themselves, besides the residue fields $\Delta$ and $\Delta^{\prime}$. 
The usefulness of the join $V^{*}$ is due to the possibility of deriving properties of the birational correspondence between $V$ and $V^{\prime}$ by first passing from $V$ to $V^{*}$ and then from $V^{*}$ to $V^{\prime}$. In each of these two steps we are dealing with a birational correspondence between two varieties which has no fundamental elements on one of the varieties (on $V^{*}$ ). Birational correspondences of this sort are easier to handle, and they in fact play an important role in the general theory and in applications.

6. Further properties of fundamental varieties.

THEOREM 12. Given an irreducible subvariety $W$ of $V$ there exists an algebraic subvariety of $V^{\prime}$ which we shall denote by $T[W]$ and which has the following properties:

A. Each irreducible component of $T[W]$ corresponds to $W$. $T[W]$.

B. Each irreducible subvariety $W^{\prime}$ of $V^{\prime}$ which corresponds to $W$ lies on

The variety $T[W]$ shall be referred to in the sequel as the transform of $W$.

Proof. Suppose that the theorem is true for $V$ and the join $V^{*}$ of $V$ and $V^{\prime}$. Then we show that it is also true for $V$ and $V^{\prime}$. For let $T^{*}$ denote, as before, the birational correspondence between $V$ and $V^{*}$ and let $T^{*}[W]=W_{1}^{*}+W_{2}^{*}+\cdots+W_{h}^{*}$, where each $W_{i}^{*}$ is irreducible. To each $W_{i}^{*}$ there corresponds on $V^{\prime}$ a unique irreducible variety $W_{i}^{\prime}$. Since, by hypothesis, $T^{*}(W)=W_{i}^{*}$, it follows that each of the varieties $W_{1}^{\prime}, W_{2}^{\prime}, \cdots, W_{h}^{\prime}$ corresponds to $W$. On the other hand, let $W^{\prime}$ be any irreducible subvariety of $V^{\prime}$ which corresponds to $W$, and let $W^{*}$ be an irreducible subvariety of $V^{*}$ which corresponds to both $W$ and $W^{\prime}$. By hypothesis, $W^{*} \subseteq T^{*}(W)$, say $W^{*} \subseteq W_{1}^{*}$. Passing to the corresponding subvarieties $W^{\prime}$ and $W_{1}^{\prime}$ of $V^{\prime}$, we conclude that $W^{\prime} \subseteq W_{1}^{\prime}$, that is, $W^{\prime} \subseteq W_{1}^{\prime}+W_{2}^{\prime}+\cdots+W_{h}^{\prime}$. Hence $T[W]=W_{1}^{\prime}+W_{2}^{\prime}+\cdots+W_{h}^{\prime}$, where, of course, some of the $h$ varieties $W_{i}^{\prime}$ may be embedded, so that the number of irreducible components of $T[W]$ may actually be less than $h$.

We now prove the theorem for $V$ and for the join $V^{*}$. We use nonhomogeneous coordinates. As nonhomogeneous coordinates for $V^{*}$ we can use the coordinates $\xi_{i j}$ of the preceding section, since the $(n+1)(m+1)$ systems of coordinates $\eta_{i j} / \eta_{\alpha \beta}$ ( $\alpha$ and $\beta$ are fixed for each system) cover the entire projective space in which $V^{*}$ is embedded. To prove the existence of the transform $T^{*}[W]$, it will be sufficient therefore to exhibit that part $L^{*}$ of $T^{*}[W]$ which is at finite distance with respect to the coordinates $\xi_{i j}$. Let $\mathfrak{o}^{\prime} \mathrm{o}^{\prime}$ and $\mathrm{o}^{*}$ have the same meaning as in the preceding section. If $W$ is not at finite distance with respect to the coordinates $\xi_{i}$, then no $W^{*}$ which corresponds to $W$ on $V^{*}$ can be at finite distance with respect to the coordinates $\xi_{i j}$ (since $\left.\mathfrak{D} \subseteq \mathfrak{o}^{*}\right)$. Hence in this case $L^{*}$ is empty. If $W$ is at finite distance, it is given by a prime ideal $\mathfrak{p}$ in $\mathfrak{o}$. Let $\mathfrak{p}_{1}^{*}, \mathfrak{p}_{2}^{*}, \cdots, \mathfrak{p}_{h}^{*}$ be those minimal prime ideals of $\mathfrak{o}^{*} \cdot \mathfrak{p}$ which contract to $\mathfrak{p}$, and let $W_{i}^{*}$ be the irreducible subvariety of $V^{*}$ 
which is defined by $\mathfrak{p}_{i}^{*}$. I assert that $L^{*}=W_{1}^{*}+W_{2}^{*}+\cdots+W_{h}^{*}$. For in the first place, each $W_{i}^{*}$ corresponds to $W$. In the second place, if $W^{*}=T^{*}(W)$ and if $W^{*}$ is at finite distance with respect to the coordinates $\xi_{i j}, W^{*}$ is given

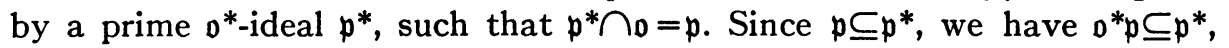
whence $\mathfrak{p}^{*}$ must divide some minimal ideal $\mathfrak{p}^{\prime *}$ of $\mathfrak{o}^{*} \mathfrak{p}$. Since $\mathfrak{p} \subseteq \mathfrak{p}^{\prime *} \subseteq \mathfrak{p}^{*}$, it follows that $\mathfrak{p} \subseteq \mathfrak{p}^{* *} \cap_{\mathfrak{D}} \subseteq \mathfrak{p}$, that is $\mathfrak{p}^{* *} \cap_{\mathfrak{D}}=\mathfrak{p}$. Therefore $\mathfrak{p}^{* *}$ is one of the ideals $\mathfrak{p}_{1}^{*}, \mathfrak{p}_{2}^{*}, \cdots, \mathfrak{p}_{h}^{*}$, and since $\mathfrak{p}^{*} \supseteq \mathfrak{p}^{*}$, we conclude that $W^{*} \subseteq W_{1}^{*}+W_{2}^{*}$ $+\cdots+W_{h}^{*}$. This completes the proof.

COROLlaRY 1. If the birational correspondence between $V$ and $V^{\prime}$ has no fundamental elements on $V^{\prime}$, then to a fundamental variety $W$ on $V$ there corresponds on $V^{\prime}$ at least one variety of higher dimension than $W$; in other words, $T[W]$ is of higher dimension than $W$.

For if there are no fundamental elements on $V^{\prime}$ and if $W^{\prime}=T(W)$ then dimension $W^{\prime} \geqq$ dimension $W$ (Theorem 8, (B) and (C), II.3). If every $W^{\prime}$ which corresponds to $W$ were of the same dimension as $W$, then $T[W]$ would be pure $\rho$-dimensional, where $\rho=$ dimension $W$. But then the irreducible components of $T[W]$ would be the only subvarieties of $V^{\prime}$ which correspond to $W$, and since the number of these components is finite, the corollary follows, by Theorem 10, II.3.

As a consequence, we have the following characterization of fundamental varieties:

COROLlary 2. If $V$ and $V^{\prime}$ are arbitrary birationally equivalent varieties and if $T$ and $T^{*}$ denote, respectively, the birational correspondence between $V$ and $V^{\prime}$ and the birational correspondence between $V$ and $V^{*}$, then a given irreducible subvariety $W$ of $V$ is fundamental for $T$ if and only if $T^{*}[W]$ is of higher di. mension than $W$.

For $W$ is fundamental for $T$ if and only if it is fundamental for $T^{*}$ (Theorem 11, II.5) and since $T^{*}$ has no fundamental elements on $V^{*}$.

In addition to the transform $T[W]$ we shall also have occasion to consider what we call the total transform of $W$ and that we shall denote by $T\{W\}$. By that we mean the locus of points of $V^{\prime}$ which correspond to points of $W$. That $T\{W\}$ is an algebraic variety is seen as follows. As in the case of $T[W]$, so also here it is sufficient to show that $T^{*}\{W\}$ is algebraic, where $T^{*}$ is, as usual, the birational correspondence between $V$ and the join $V^{*}$ of $V$ and $V^{\prime}$. Now if we consider that part $L_{1}^{*}$ of $T^{*}\{W\}$ which is at finite distance with respect to the nonhomogeneous coordinates $\xi_{i j}$ we see immedi. ately that $L_{1}^{*}$ is the algebraic subvariety of $V^{*}$ which is defined by the ideal $0^{*} \cdot p$. For a point $P^{*}$ of $V^{*}$, at finite distance with respect to the $\xi_{i j}$ 's, corresponds to a point $P$ on $W$, if and only if the corresponding 0 -dimensional prime $\mathfrak{p}^{*}$-ideal $\mathfrak{p}^{*}$ satisfies the relation: $\mathfrak{p}^{*} \cap \mathfrak{D} \supseteq \mathfrak{p}$, that is, if and only if $\mathfrak{p}^{*} \supseteq \mathfrak{p}^{*} \cdot \mathfrak{p}$.

The irreducible components of $T^{*}\{W\}$, at finite distance, correspond to 
the minimal prime ideals of $\boldsymbol{D}^{*} \cdot \mathfrak{p}$. We have seen that the irreducible components of $T^{*}[W]$, at finite distance, correspond to those minimal prime ideals of $\mathfrak{D}^{*} \mathfrak{p}$ which contract to $\mathfrak{p}$. Hence $T^{*}[W]$ lies on $T^{*}\{W\}$, and from this follows immediately that also $T[W]$ lies on $T\{W\}$. This result can also be deduced directly from property $B$, stated in II.1. In the same fashion one sees immediately that $T\{W\}$ also has the following property: If $W_{1} \subseteq W$, and if $W_{1}^{\prime}=T\left(W_{1}\right)$, then $W_{1}^{\prime} \subseteq T\{W\}$.

We point out explicitly that $T[W]$ may very well be a proper subvariety of $T\{W\}$. For instance, if $T$ is a plane Cremona transformation and if $W$ is a curve containing a fundamental point $P$, then $T\{W\}=T[W]+\Gamma^{\prime}$, where $\Gamma^{\prime}$ is the irregular ("fundamental" in the old terminology) curve which corresponds to the point $P$. Here $T[W]$ is either a curve (if $W$ is regular) or a fundamental point (if $W$ is irregular) $\left({ }^{26}\right)$. Quite generally, we have the following theorem:

TheOREM 13. Any irreducible component of the total transform $T\{W\}$ which is not a component of the transform $T[W]$ must correspond to a proper subvariety $W_{1}$ of $W$. Moreover, if $V$ is locally normal, then $W_{1}$ must be a fundamental variety.

Proof. In the proof of Theorem 12 we have seen that if $T^{*}[W]$ $=W_{1}^{*}+W_{2}^{*}+\cdots+W_{h}^{*}$ and if $W_{i}^{\prime}$ is the subvariety of $V^{\prime}$ which corresponds to $W_{i}^{*}$, then $T[W]=W_{1}^{\prime}+W_{2}^{\prime}+\cdots+W_{h}^{\prime}$. In a similar fashion it is seen immediately that if $T^{*}\{W\}=W_{1}^{*}+W_{2}^{*}+\cdots+W_{h}^{*}+W_{h+1}^{*}+\cdots$, then $T\{W\}=W_{1}^{\prime}+W_{2}^{\prime}+\cdots+W_{h}^{\prime}+W_{h+1}^{\prime}+\cdots$. Now let $W_{0}^{\prime}$ be an irreducible component of $T\{W\}$ which does not belong to $T[W]$. Then $W_{0}^{\prime}$ must correspond to an irreducible component $W_{0}^{*}$ of $T^{*}\{W\}$ which does not belong to $T^{*}[W]$. Now by definition of $T^{*}\{W\}$, each point of $W_{0}^{*}$ must correspond to some point of $W$. Since to a point of $V^{*}$ there corresponds a unique point of $V$, it follows that the subvariety $W_{0}$ of $V$ which corresponds to $W_{0}^{*}$ must lie on $W$. It must be a proper subvariety of $W$, since $W_{0}^{*} \supseteq T^{*}[W]$. Now both $W_{0}^{\prime}$ and $W_{0}$ correspond to $W_{0}^{*}$, whence they correspond to each other. It remains to prove that $W_{0}$ is fundamental for $T$. If $W_{0}$ were not funda-

${ }^{(26)}$ This example shows therefore that the ideal $\mathfrak{D}^{*} \mathfrak{p}$ may very well possess minimal prime ideals which contract in $\mathbf{D}$ (not to $\mathfrak{p}$ but) to proper divisors of $\mathfrak{p}$. In this connection we wish to correct a statement on p. 135 in Krull's Ergebnisse report Idealtheorie. The theorem stated on that page consists of three parts, and in the first part it is asserted, among other things, that $\tilde{\mathfrak{p}}=\mathfrak{p} \cdot \tilde{\mathfrak{\Im}}$. This (and only this) assertion is incorrect. The rings $\Im$ and $\widetilde{\Im}$ play there the role of our rings 0 and $0^{*}$. It is quite true that there is only one prime ideal $\tilde{\mathfrak{p}}$ which lies over $\mathfrak{p}$ (that is, such that $\tilde{\mathfrak{p}} \cap \Im=\mathfrak{p})$, and it is also true that $\mathfrak{p}$ is a minimal prime of the ideal $\tilde{\Im} \cdot \mathfrak{p}$. The equality $\widetilde{\Im}_{\tilde{p}}=\Im_{\mathfrak{p}}$ shows clearly that the case under consideration corresponds to a regular $W$. Nevertheless, $\widetilde{\Im} \cdot \mathfrak{p}$ may have minimal prime ideals other than $\tilde{\mathfrak{p}}$, as was pointed out above. Already the quadratic transformation $x^{\prime}=x, y^{\prime}=y / x$ may serve very well as a source of simple counterexamples. The formal source of that incorrect statement made in Krull's report is the erroneous assertion made earlier on the same page (line 12) to the effect that if the rank equals $m$ then $L_{\mathfrak{p}}$ is a field. 
mental, then by a stronger reason $W$ would not be fundamental, since $Q(W) \supset Q\left(W_{0}\right)$ (see Theorem $8(\mathrm{E})$ ). But then we would have $T\left[W_{0}\right] \subset T[W]$, a contradiction.

If $V$ is not locally normal, one passes to the derived normal varieties of $V$ and of $V^{*}$ and the rest of the proof is straightforward.

COROLlARY. If no subvariety of $W$ is fundamental, and if $V$ is locally normal, then the total transform of $W$ coincides with the transform of $W$.

We make one more remark about the transforms $T[W]$ and $T\{W\}$. It is clear that in no case can these varieties be empty. However, it may very well happen that with respect to a given system of nonhomogeneous coordinates either $T[W]$ or even $T\{W\}$ is entirely at infinity. Referring to the join $V^{*}$ and to the rings $\boldsymbol{D}$ and $\boldsymbol{D}^{*}$ considered above, we see that $T^{*}[W]$ is at infinity if $\mathrm{D}^{*}$ does not contain prime ideals which contract to $\mathfrak{p}$ (in the terminology of Krull: $\mathfrak{p}$ is lost in $\mathfrak{o}^{*}$, see [2, p. 134]). If also $T^{*}\{W\}$ is entirely at infinity, then the ideal $\mathrm{D}^{*} \cdot \mathrm{p}$ is the unit ideal.

7. The main theorem. If the birational correspondence between $V$ and $V^{\prime}$ has no fundamental elements on $V^{\prime}$, and if $W$ is fundamental for $T$, then, by Theorem 12, Corollary 1 , the transform $T[W]$ has at least one component of higher dimension than $W$. In the general case, that is, when $V$ is an arbitrary variety, that is the best result one may claim, since it is quite possible for $T[W]$ to possess components which have the same dimension as $W$. However, in the case in which $V$ is locally normal at $W$ we have the following important theorem:

MAIN THEOREM. If $W$ is an irreducible fundamental variety on $V$ of a birational correspondence $T$ between $V$ and $V^{\prime}$ and if $T$ has no fundamental elements on $V^{\prime}$, then-under the assumption that $V$ is locally normal at $W$-each irreducible component of the transform $T[W]$ is of higher dimension than $W$.

COROLlaRy. In the more general case in which $T$ has fundamental elements on both $V$ and $V^{\prime}$ and under the assumption that $W$ is fundamental for $T$ and that $V$ is locally normal at $W$, the transform $T^{*}[W]$ of $W$ on the join $V^{*}$ of $V$ and $V^{\prime}$ has the property that all its irreducible components are of higher dimension than $W$.

We shall first give the main theorem another formulation which is more directly algebraic. Since $V$ is locally normal at $W$, it is permissible to replace $V$ by a derived normal variety of $V$ (Theorem 7, Corollary 2, II.2). Hence we assume that $V$ is a normal variety. Since there are no fundamental elements on $V^{\prime}$, the birational correspondence between $V^{\prime}$ and $V^{*}$ is regular (Theorem 11, II.5). Hence it is permissible to replace $V^{\prime}$ by $V^{*}$. When these preparations are carried out then the main theorem expresses a feature of the the relationship between the ideals in the two rings $\mathfrak{D}$ and $\boldsymbol{D}^{*}$ considered in II.5, that is, we have to prove the following theorem: 
TheOREM 14. Let $\mathrm{D}$ and $\mathrm{D}^{*}$ be two finite integral domains with the same quotient field $\Sigma$, where we assume that the ring $\mathrm{o}$ is integrally closed and that it is a subring $\left({ }^{27}\right)$ of $\mathrm{o}^{*}$. Let $\mathrm{p}$ be a prime ideal in $\mathrm{o}$ and let $\mathrm{p}^{*}$ be a prime ideal $\mathrm{o}^{*}$ which lies over $\mathfrak{p}$, that is, such that $\mathfrak{p}^{*} \cap_{\mathfrak{D}}=\mathfrak{p}$. If $\mathfrak{p}$ and $\mathfrak{p}^{*}$ have the same dimension, then

(1) either the quotient rings $\mathfrak{o}_{\mathfrak{p}}$ and $\mathfrak{o}_{\mathfrak{p}^{*}}^{*}$ coincide ${ }^{28}$ ) (and in this case $\mathfrak{p}^{*}$ is obviously the only prime $\mathfrak{D}^{*}$-ideal which lies over $\left.\mathfrak{p}\right)$ or

(2) $\mathfrak{p}^{*}$ is not minimal with respect to the property of lying over $\mathfrak{p}$, that is, there exists in $\mathrm{D}^{*}$ another prime ideal which also lies over $\mathrm{p}$ and which is a proper multiple of $\mathrm{p}^{*}$.

The proof of this theorem is rather long and will be developed in this and in the following section. That part of the proof which is contained in this section consists of three steps: (a) a reduction to a simpler special case; (b) a reference to the theorem of Krull which we have already mentioned( $\left.{ }^{26}\right)$; (c) a lemma.

(a) Let $\mathfrak{o}^{* *}$ be the integral closure of $\mathrm{o}^{*}$ in $\Sigma$. It is well known that over every prime ideal $\mathfrak{p}^{*}$ in $\mathfrak{D}^{*}$ there lies at least one prime ideal $\mathfrak{p}^{\prime *}$ in $\mathfrak{o}^{\prime *}$, and that the number of such ideals $\mathfrak{p}^{* *}$ is finite. Moreover $\mathfrak{p}^{*}$ and $\mathfrak{p}^{\prime *}$ have the same dimension and it is clear that if $p^{*} \cap \mathfrak{D}=\mathfrak{p}$, then $\mathfrak{o}_{\mathfrak{p}} \subseteq \mathfrak{o}_{p^{*}}^{*} \subseteq \mathfrak{o}_{p^{* *}}^{\prime *}$. From this it follows that if our theorem is true for the pair of rings 0 and $\boldsymbol{D}^{\prime *}$, then it is also true for $\mathrm{D}$ and $\mathrm{o}^{*}$. Hence we may assume that $\mathrm{D}^{*}$ is integrally closed.

Since $\mathfrak{D}$ and $\mathfrak{o}^{*}$ are finite integral domains, $\mathfrak{o}^{*}$ is a finite ring extension of $\boldsymbol{o}$. We prefer to think of $\mathrm{o}^{*}$ as a ring obtained from $\mathrm{o}$ by a finite number of simple ring extensions, each ring extension being followed up by the operation of integral closure. Let therefore:

$$
\begin{aligned}
& \mathfrak{o}_{1}=\mathfrak{o}\left[\alpha_{1}\right], \mathfrak{o}_{1}^{\prime}=\text { integral closure of } \mathfrak{o}_{1} ; \\
& \mathrm{D}_{2}=\mathrm{D}_{1}^{\prime}\left[\alpha_{2}\right], \mathrm{D}_{2}^{\prime}=\text { integral closure of } \mathrm{D}_{2} ; \\
& \mathfrak{D}_{m}=\mathfrak{o}_{m-1}^{\prime}\left[\alpha_{m}\right], \mathfrak{o}_{m}^{\prime}=\mathfrak{o}^{*}=\text { integral closure of } \mathfrak{o}_{m} \text {. }
\end{aligned}
$$

Let us assume that our theorem is true when $m=1$. Then we show, by induction with respect to $m$, that the theorem is true for any value of $m$. Let $\mathfrak{p}^{*} \cap \mathfrak{o}_{m-1}^{\prime}=\mathfrak{p}_{m-1}^{\prime}$. Since $\mathfrak{p}_{m-1}^{\prime} \cap \mathfrak{D}=\mathfrak{p}$, we have: dimension $\mathfrak{p}^{*} \geqq$ dimension $\mathfrak{p}_{m-1}^{\prime} \geqq$ dimension $\mathfrak{p}$. If $\mathfrak{p}$ and $\mathfrak{p}^{*}$ have the same dimension, then it follows that also $\mathfrak{p}_{m-1}^{\prime}$ has the same dimension as $\mathfrak{p}$. Now let us also assume that no proper prime multiple of $\mathfrak{p}^{*}$ lies over $\mathfrak{p}$. Then no proper prime multiple of $\mathfrak{p}^{*}$ can lie over $\mathfrak{p}_{m-1}^{\prime}$, and therefore, by the case $m=1$, we have that $\mathfrak{o}_{p^{*}}^{*}$ coincides with the quotient ring of $\mathfrak{p}_{m-1}^{\prime}$ in $\mathrm{D}_{m-1}^{\prime}$. Consequently, there is a $(1,1)$ correspondence between the prime multiples of $p^{*}$ in $\mathfrak{o}^{*}$ and the prime multiples

(27) It will be seen from the proof that these assumptions can be weakened as follows: o has a finite degree of transcendency over the ground field and is a finite discrete principal order (see Krull [2, p. 104]); $\mathrm{D}^{*}$ is a finite ring extension of $\mathrm{D}$.

${ }^{(28)}$ This case arises when $W$ is not fundamental, therefore regular. 
of $\mathfrak{p}_{m-1}^{\prime}$ in $\mathfrak{D}_{m-1}^{\prime}$. (See I.2.) Therefore it is equally true that no prime multiple of $\mathfrak{p}_{m-1}^{\prime}$ lies over $\mathfrak{p}$. By our induction, we conclude that also $\mathfrak{D}_{\mathfrak{p}}$ coincides with the quotient ring of $\mathfrak{p}_{m-1}^{\prime}$ in $\mathrm{D}_{m-1}^{\prime}$. Hence $\mathrm{o}_{\mathfrak{p}}=\mathrm{o}_{\mathfrak{p}}^{*}$, as was asserted.

We therefore have only to prove our theorem in the following special case: $\mathrm{D}^{*}$ is the integral closure of a ring $\mathrm{D}^{\prime}$, where $\mathrm{D}^{\prime}$ is a simple ring extension of $\mathrm{D}$ : $\mathrm{o}^{\prime}=\mathrm{o}[\alpha]$.

(b) In this special case we shall make use of a theorem stated in Krull $[2$, p. 135$]$, to which we have already referred in the preceding section $\left({ }^{26}\right)$. We write the principal fractional ideal $\boldsymbol{D} \cdot \alpha$ as a quotient of two integral ideals: $\mathfrak{D} \cdot \alpha=\mathfrak{z} / \mathfrak{n}$, where $z$ and $\mathfrak{n}$ are symbolic power products of minimal prime ideals in $\mathfrak{D}$, without common factors. Krull distinguishes three cases: (1) $\mathfrak{n} \equiv 0(\mathfrak{p}), \quad z \neq 0(p) ;(2) \mathfrak{n} \neq \equiv 0(\mathfrak{p}) ;(3) \mathfrak{n} \equiv 0(\mathfrak{p}), z=0(p)$.

In the first case the element $1 / \alpha$ is a non-unit in $\mathfrak{o}_{p}$ and from this it follows immediately that $\mathfrak{p}$ is lost in $\mathfrak{o}^{\prime}$, that is, no prime ideal in $\mathfrak{o}^{\prime}$ contracts to $\mathfrak{p}$. But then $\mathfrak{p}$ is also lost in $\mathfrak{o}^{*}$, since $\mathfrak{o}^{*}$ is the integral closure of $\mathfrak{o}^{\prime}$, and in this case there is nothing to prove.

In the second case $\alpha$ is contained in the quotient ring $\mathfrak{o}_{\mathfrak{p}}$, whence $\mathfrak{o}^{*} \subset \mathrm{o}_{\mathfrak{p}}$, since $\mathfrak{D}_{\mathfrak{p}}$ is integrally closed. From this we conclude that the two rings $\mathfrak{D}_{\mathfrak{p}}$, $0_{p^{*}}^{*}$ coincide $\left({ }^{29}\right)$. This is the alternative (1) of the theorem.

The really significant case is the third one. In this case Krull's result is to the effect that $\mathfrak{p}^{\prime} \cdot \mathfrak{p}$ is a prime ideal $\mathfrak{p}^{\prime}$, that $\mathfrak{p}^{\prime}$ lies over $\mathfrak{p}$ and that the dimension of $\mathfrak{p}^{\prime}$ is one greater than the dimension of $\mathfrak{p}$. We shall make use of this result.

(c) In addition to the above result which concerns the relationship between the ideal theory in $\mathfrak{D}$ and in $\boldsymbol{D}^{\prime}$, we shall have to make use, in a very essential fashion, of the following property of the conductor $\mathfrak{S}_{\mathfrak{S}}$ of $\mathfrak{D}^{\prime}$ with respect to $0^{*}$ :

LEMMA 6. Each prime $\mathrm{D}^{*}$-ideal $\mathfrak{p}^{*}$ of the conductor $\sqrt{5}$ has the property that it contracts in $\mathrm{o}$ to a prime ideal of lower dimension.

Proof. The lemma implies in particular that 5 has no zero-dimensional prime ideals. Let us assume that this particular consequence of the lemma has been established and let us show that then the lemma follows by the usual device of ground field extension.

Let $\mathfrak{p}^{*}$ be a prime $\mathfrak{p}^{*}$-ideal and let $\mathfrak{p}^{*} \bigcap_{\mathfrak{D}}=\mathfrak{p}$. We assume that $\mathfrak{p}$ and $\mathfrak{p}^{*}$ have the same dimension, say dimension $s$. It shall now be shown that $\mathfrak{p}^{*}$ cannot be a prime ideal of $\mathfrak{S}$.

We select in $\mathrm{D}$ a set of $s$ elements $\zeta_{1}, \zeta_{2}, \cdots, \zeta_{s}$ which are algebraically independent modulo $\mathfrak{p}$. We adjoin these elements to the ground field $\mathrm{K}$ getting a new ground field $K_{1}=K\left(\zeta_{1}, \zeta_{2}, \cdots, \zeta_{s}\right)$ and also the new rings: $D_{1}=K_{1} \cdot D$, $\mathrm{o}_{1}^{\prime}=\mathrm{K}_{1} \cdot \mathrm{o}^{\prime}, \mathrm{o}_{1}^{*}=\mathrm{K}_{1} \cdot \mathrm{o}^{*}$. We point out that $\mathrm{o}_{1}^{*}$ is a quotient ring of $\mathrm{o}^{*}$, namely $\mathrm{o}_{1}^{*}=\mathrm{o}_{S}^{*}$, where $S$ is the set of all polynomials in $\zeta_{1}, \zeta_{2}, \cdots, \zeta_{s}$, with coeffi-

${ }^{(29)}$ The proof is exactly the same as the proof which in II.2 led us to the conclusion that the quotient ring of $\mathfrak{m}_{i}^{*}$ in the ring $\mathfrak{\Im}^{*}$ coincides with $\mathfrak{\Im}_{i}^{\prime}$. 
cients in K. Similarly we have: $D_{1}=o_{S}$. We therefore can apply the properties of the correspondence between the ideals in a given ring $R$ and a quotient ring $R_{S}$, as described in I.1. We find then that $\mathfrak{p}_{1}=\mathfrak{p}_{1} \cdot \mathfrak{p}$ and $\mathfrak{p}_{1}^{*}=\mathfrak{p}_{1}^{*} \cdot \mathfrak{p}^{*}$ are

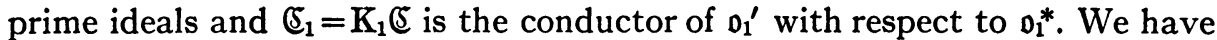
$\mathfrak{o}_{1}^{\prime}=\mathfrak{o}_{1}[\alpha]$ and it is clear that $\mathfrak{o}_{1}^{*}$ is the integral closure of $\mathfrak{o}_{1}^{\prime}$. Since $p_{1}^{*}$ is zerodimensional over the ground field $\mathrm{K}_{1}$, it follows, by our assumption, that $\mathfrak{G}_{1}: \mathfrak{p}_{1}^{*}=\mathfrak{G}_{1}$. On the other hand we have $\mathfrak{G}_{1}: \mathfrak{p}_{1}^{*}=\mathrm{K}_{1} \cdot\left(\mathfrak{S}: \mathfrak{p}^{*}\right)$. Hence the two ideals $\mathbb{E}$ and $\mathbb{(}: \mathfrak{p}^{*}$ have the same extended ideal in $\mathrm{o}_{1}^{*}$. It follows (see II.1( $\left.{ }^{3}\right)$ ) that these two ideals can only differ by primary components whose associate prime ideals contain polynomials in $\zeta_{1}, \zeta_{2}, \cdots, \zeta_{8}$. Since the $\zeta^{\prime}$ 's are algebraically independent modulo $\mathfrak{p}^{*}$, we conclude that $\mathfrak{p}^{*}$ is not among the prime ideals of $\mathfrak{E}$, as was asserted.

The proof of the lemma is thus reduced to the matter of proving that the conductor $\mathbb{S}$ does not possess zero-dimensional prime ideals.

Let $\mathfrak{p}^{*}$ be a zero-dimensional prime ideal in $\mathfrak{o}^{*}$. We have to prove that $\mathfrak{c}: \mathfrak{p}^{*}=\mathfrak{c}$. Let $\zeta^{*}$ be an arbitrary element of $\mathfrak{c}: \mathfrak{p}^{*}$. We denote by $f(x)$ the irreducible polynomial in $K[x]$ such that $f(\alpha) \equiv 0\left(p^{*}\right)$. We have then: $\zeta^{*} f(\alpha) \equiv 0(\mathcal{E})$, whence $\zeta^{*} f(\alpha) \cdot \eta^{*} \in \mathfrak{o}^{\prime}$, for any element $\eta^{*}$ in $\mathrm{o}^{*}$. Hence we may write

$$
\zeta^{*} \eta^{*} \cdot f(\alpha)=G(\alpha)=\omega_{0} \alpha^{\nu}+\omega_{1} \alpha^{\nu-1}+\cdots+\omega_{\nu}, \quad \omega_{i} \in \mathbf{0} .
$$

We divide through $G(x)$ by $f(x)$ :

$$
G(x)=A(x) f(x)+R(x),
$$

where all polynomials are in $\mathfrak{D}[x]$ and where $R(x)$ is of degree at most $m-1$, if $m$ is the degree of $f(x)$. We now rewrite (7) as follows (notice that $f(\alpha) \neq 0$, since $\alpha$ is not in $\mathcal{D}$ and since $\mathfrak{D}$ is integrally closed):

$$
\zeta^{*} \eta^{*}=A(\alpha)+\frac{R(\alpha)}{f(\alpha)} .
$$

Let $v$ be an arbitrary valuation of $\Sigma$ whose valuation ring $R_{v}$ contains $\boldsymbol{D}$. If $v(\alpha) \geqq 0$, then $\mathfrak{o}[\alpha] \in R_{v}$, and also $0^{*} \subset R_{v}$. Hence, by (8), we have $R(\alpha) / f(\alpha) \in R_{v}$. If $v(\alpha)<0$, then $v(R(\alpha))>v(f(\alpha))$, since $R$ is of less degree than $f$ and since $v(f(\alpha))=m v(\alpha)$ (the leading coefficient of $f(\alpha)$ is an element of $\mathrm{K}$ ). Hence also in this case $R(\alpha) / f(\alpha)$ is contained in $R_{v}$. Since this holds true for any valuation $v$ such that $\mathfrak{D} \subset R_{v}$ and since $\mathrm{D}$ is integrally closed, we conclude that $R(\alpha) / f(\alpha) \in \mathrm{o}$. Hence, by $(8), \zeta^{*} \eta^{*} \in \mathrm{o}^{\prime}$, for any element $\eta^{*}$ in $\mathrm{o}^{*}$. Consequently $\zeta^{*} \in \mathfrak{C}$. Since $\zeta^{*}$ was an arbitrary element of $\mathfrak{E}: \mathfrak{p}^{*}$, it follows that $\mathfrak{E}: \mathfrak{p}^{*}=\mathfrak{C}$. This completes the proof of the lemma.

8. Continuation of the proof of the main theorem. To prove the main theorem, or better, the equivalent Theorem 14, we shall proceed as follows. We assume that we have the special case described in the preceding section under (a). Let $\mathfrak{p}^{*}$ be a prime ideal in $\mathfrak{o}^{*}$ and let $\mathfrak{p}^{*} \cap \mathfrak{D}=\mathfrak{p}$. We shall also assume 
that we are dealing with the significant case $\mathfrak{n} \equiv 0(\mathfrak{p}), z \equiv 0(\mathfrak{p})$, in which case we have the result of Krull as stated in the preceding section under (b). We shall prove that if $p$ and $p^{*}$ have the same dimension, then $p^{*}$ contains properly another prime ideal $\mathfrak{p}_{1}^{*}$ with the property: $\mathfrak{p}_{1}^{*} \bigcap_{\mathfrak{D}}=\mathfrak{p}$. This is the second alternative of Theorem 14 .

We divide the proof into two parts, according as $\mathfrak{E} \not \equiv 0\left(p^{*}\right)$ or $\mathfrak{C} \equiv 0\left(p^{*}\right)$.

First case: $\mathbb{E} \not \equiv 0\left(\mathfrak{p}^{*}\right)$. Let $\mathfrak{p}^{*} \cap \mathfrak{o}^{\prime}=\mathfrak{p}_{1}^{\prime}$. Since $\mathfrak{C} \neq 0\left(\mathfrak{p}_{1}^{\prime}\right)$, it follows in an elementary fashion from the very definition of the conductor, that $p^{*}$ is the only prime ideal in $D^{*}$ which contracts to $p_{1}^{\prime}$ and that the quotient rings $\mathfrak{o}_{\mathfrak{p}^{*}}^{*}, \mathfrak{o}_{\mathfrak{p}_{1}^{\prime}}^{\prime}$ coincide. We have $\mathfrak{p}_{1}^{\prime} \cap \mathfrak{D}=\mathfrak{p}$, whence $\mathfrak{p}_{1}^{\prime}$ must be a divisor of the prime ideal $\mathfrak{p}^{\prime}=\mathfrak{p}^{\prime} \cdot \mathfrak{p}$. It must be a proper divisor of $\mathfrak{p}^{\prime}$, since $\mathfrak{p}_{1}^{\prime}$ is of the same dimension as $\mathfrak{p}$, while by Krull's result $\mathfrak{p}^{\prime}$ is of dimension one greater than $\mathfrak{p}$. Now

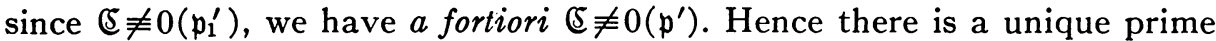
ideal $\mathfrak{p}^{\prime *}$ in $\mathfrak{o}^{*}$ which contracts to $\mathfrak{p}^{\prime}$ and we have $\mathfrak{o}_{\mathfrak{p}^{*}}^{*}=\mathfrak{o}_{\mathfrak{p}^{\prime}}^{\prime}$. Since we have also $\mathfrak{p}_{\mathfrak{p}^{*}}^{*}=\mathfrak{o}_{\mathfrak{p}^{\prime} 1}^{\prime}$ and since $\mathfrak{p}_{1}^{\prime}$ is a proper divisor of $\mathfrak{p}^{\prime}$, it follows that also $\mathfrak{p}^{*}$ is a proper divisor of $\mathfrak{p}^{* *}$. Since $\mathfrak{p}^{*} \cap \mathfrak{D}=\mathfrak{p}^{\prime} \cap \mathfrak{D}=\mathfrak{p}$, our proof is complete.

Second case: $\mathfrak{E} \equiv 0\left(\mathfrak{p}^{*}\right)$. In this case $\mathfrak{p}^{*}$ is either a prime ideal of the conductor $\mathbb{E}$ or properly contains a prime ideal of $\mathfrak{C}$. Since, by hypothesis, $\mathfrak{p}^{*}$ contracts in $\mathfrak{D}$ to an ideal $\mathfrak{p}$ of the same dimension as $\mathfrak{p}^{*}$, the first possibility is excluded by our lemma. Hence $\mathfrak{p}^{*}$ properly contains a prime ideal $\mathfrak{p}_{1}^{*}$ of $\mathfrak{c}$. Let $\mathfrak{p}_{1}^{*} \cap_{\mathfrak{o}}=\mathfrak{p}_{1}$ and let

$$
\text { dimension } \mathfrak{p}=s, \quad \text { dimension } \mathfrak{p}_{1}=s_{1} \text {. }
$$

Since $\mathfrak{p}^{*} \supset \mathfrak{p}_{1}^{*}$, we have $\mathfrak{p} \supseteq \mathfrak{p}_{1}$. If $\mathfrak{p}_{1}=\mathfrak{p}$ then the theorem is proved, since we have now a proper multiple $\mathfrak{p}_{1}^{*}$ of $\mathfrak{p}^{*}$ which also contracts to $\mathfrak{p}$. We assume therefore that $p \supset p_{1}$, whence $s_{1}>s$. By our lemma, $\mathfrak{p}_{1}^{*}$ is of greater dimension than $\mathfrak{p}_{1}$; by Krull's theorem, the dimension of $\mathfrak{p}_{1}^{*}$ is at most one greater than the dimension of $p_{1}$. Hence the dimension of $p_{1}^{*}$ is $s_{1}+1$. Our proof would be complete if we could show that there exists a prime ideal in $0^{*}$, between $p^{*}$ and $\mathfrak{p}_{1}^{*}$ (and different from $\mathfrak{p}^{*}$ ) which contracts to $\mathfrak{p}$. This we proceed to show.

We pass to the residue class rings $\mathfrak{D}=\mathfrak{D} / \mathfrak{p}_{1}, \mathfrak{D}^{*}=\mathfrak{D}^{*} / \mathfrak{p}_{1}^{*}$. Both rings are finite integral domains and $\mathfrak{D}$ is a subring of $\mathfrak{D}^{*}$. The first ring is of degree of transcendency $s_{1}$, while $\mathfrak{D}^{*}$ is of degree of transcendency $s_{1}+1$. In the homomorphisms $\mathfrak{0} \sim \mathfrak{D}_{1} \mathfrak{o}^{*} \sim \mathfrak{D}^{*}$, the prime ideals $\mathfrak{p}$ and $\mathfrak{p}^{*}$ are mapped, respectively, onto prime ideals $\mathfrak{P}$ and $\mathfrak{P}^{*}$, of the same dimension $s, s<s_{1}$, and we have: $\mathfrak{B}^{*} \cap \mathfrak{D}=\mathfrak{B}$. What we have to prove is the existence in $\mathfrak{D}^{*}$ of a prime ideal which is a proper multiple of $\mathfrak{P}^{*}$ and which contracts in $\mathfrak{D}$ to the ideal $\mathfrak{P}$. The assertion that such an ideal exists is equivalent to the assertion that $\mathfrak{B}^{*}$ is not a minimal prime of the extended ideal $\mathfrak{D}^{*} \cdot \mathfrak{B}$. To prove this we pass to the quotient rings $\Im=\mathfrak{O}_{\mathfrak{B}}, \Im^{*}=\mathfrak{O}_{\mathfrak{P}^{*}}^{*}$. If $\mathfrak{m}$ and $\mathfrak{m}^{*}$ denote the ideals of non-units in these two rings, then our problem is to prove that $\mathfrak{m}^{*}$ is not a minimal prime ideal of the extended ideal $\Im^{*} \cdot \mathfrak{m}$. The proof of this will complete the proof of the main theorem. 
Since $\Im$ is of degree of transcendency $s_{1}$, we can find $s_{1}-s$ elements $\zeta_{1}, \zeta_{2}, \cdots, \zeta_{s_{1-8}}$ in $\Im$ such that the ideal $\mathfrak{A}=\Im \cdot\left(\zeta_{1}, \zeta_{2}, \cdots, \zeta_{s_{1-s}}\right)$ be exactly $s$-dimensional. Since the ideal of non-units $\mathfrak{m}$ in $\Im$ is also $s$-dimensional, it follows that $\mathfrak{A}$ will then be necessarily a primary ideal, with $\mathfrak{m}$ as associated prime. Now consider the ideal $\mathfrak{A}^{*}=\Im^{*} \cdot\left(\zeta_{1}, \zeta_{2}, \cdots, \zeta_{s_{1-8}}\right)$. Since $\Im^{*}$ is of degree of transcendency $s_{1}+1$ and since $\mathfrak{A}^{*}$ is not the unit ideal (since $\zeta_{i} \in \mathfrak{A} \equiv 0(\mathfrak{m})$, whence $\mathfrak{A}^{*} \equiv 0\left(\mathfrak{m}^{*}\right)$ ), every minimal prime of $\mathfrak{U}^{*}$ is of dimension at least $s+1$. Let $\mathfrak{P}^{*}$ be a minimal prime of $\mathfrak{A}^{*}$. Since $\mathfrak{H}^{*} \equiv 0\left(\mathfrak{P}^{*}\right)$, we have $\mathfrak{B}^{*} \cap \mathfrak{Y} \supseteq \mathfrak{A}$, whence $\mathfrak{B}^{*} \cap \mathfrak{F}=\mathfrak{m}$, for $\mathfrak{A}$ is primary and its associated prime is the ideal $m$ of non-units. Hence $\mathfrak{P}^{*} \supseteq \mathfrak{S}^{*} \mathfrak{m}$, and this shows that $\mathfrak{m}^{*}$ is not a minimal prime of $\mathfrak{\Im}^{*} \cdot \mathfrak{m}$, since $\mathfrak{B}^{*}$ is a proper multiple of $\mathfrak{m}^{*}$ (dimension $\mathfrak{m}^{*}=s$, dimension $\mathfrak{B}^{*} \geqq s+1$ ), q.e.d.

In the main theorem we have assumed that the birational correspondence has no fundamental elements on $V^{\prime}$. In the general case of an arbitrary pair of birationally equivalent varieties $V$ and $V^{\prime}$ we may apply the main theorem to $V$ and to the join $V^{*}$ of $V$ and $V^{\prime}$. If we then take into account Theorem 11 of II.5 we deduce the following corollary which expresses the local character of the main theorem:

Corollary. If $W$ is an irreducible subvariety of $V$ at which $V$ is locally normal and if $W$ is fundamental for the birational correspondence $T$ between $V$ and some other variety $V^{\prime}$, then each irreducible component of $T[W]$ which is not fundamental for $T^{-1}$ is of higher dimension than $W$.

9. The fundamental locus of a birational correspondence. The forms $\phi_{0}(\eta), \phi_{1}(\eta), \cdots, \phi_{m}(\eta)$ which are proportional to the coordinates $\eta_{0}^{\prime}, \eta_{1}^{\prime}$, $\cdots, \eta_{m}^{\prime}$ of the general point of $V^{\prime}$ (see equations (4), II.5) define a linear system of forms:

$$
\phi_{\lambda}=\lambda_{0} \phi_{0}+\lambda_{1} \phi_{1}+\cdots+\lambda_{m} \phi_{m}
$$

We shall allow the parameters $\lambda_{i}$ to take arbitrary values (not all zero) in the relative algebraic closure $K^{\prime}$ of $K$ in $\Sigma$, that is, the $\lambda$ 's shall be elements of $\Sigma$ which are either in $\mathrm{K}$ or algebraic over $\mathrm{K}$. We shall also assume that $V$ and $V^{\prime}$ are locally normal varieties. Under this assumption it is permissible to identify $\mathrm{K}$ with $\mathrm{K}^{\prime}$, since any ring of nonhomogeneous coordinates of the general point of a locally normal variety is integrally closed and consequently contains $\mathrm{K}^{\prime}$. We therefore assume that $\mathrm{K}$ itself is algebraically closed in $\Sigma$.

The principal ideal $\left(\phi_{\lambda}\right)$ in the ring $K\left[\eta_{0}, \eta_{1}, \cdots, \eta_{m}\right]$ is $(r-1)$-dimensional. Since $V$ is locally normal, the conductor of this ring with respect to its integral closure is a primary irrelevant ideal (or the unit ideal). Hence, to within an irrelevant component which we shall disregard, the ideal $\left(\phi_{\lambda}\right)$ is quasi-gleich to a product of symbolic powers of minimal prime (homogeneous) ideals. In particular, let 


$$
\left(\phi_{i}\right)=\mathfrak{M N}_{i},
$$$$
i=0,1, \cdots, m,
$$

where $\mathfrak{A}_{0}, \mathfrak{A}_{1}, \cdots, \mathfrak{A}_{m}$ have no common factor. Then $\mathfrak{M}$ will be the h.c.d. of all principal ideals $\left(\phi_{\lambda}\right)$, that is, we will have: $\left(\phi_{\lambda}\right)=\mathfrak{M} \cdot \mathfrak{A}_{(\lambda)}$. The ideal $\mathfrak{A}_{(\lambda)}$ defines a pure $(r-1)$-dimensional subvariety $\mathfrak{S}_{(\lambda)}$ of $V$, which may be reducible and in which each irreducible component is counted to a definite multiplicity (equal to the exponent of the corresponding prime factor of $\left.\mathfrak{A}_{(\lambda)}\right)$. As the $\lambda$ 's vary in $\mathrm{K}$, the variety $C_{(\lambda)}$ varies and describes a linear system $|C|$ of $(r-1)$ dimensional varieties on $V$, free from fixed components since $\mathfrak{A}_{0}, \mathfrak{A}_{1}, \cdots, \mathfrak{A}_{m}$ have no common factor. We have in particular the members $C_{0}, C_{1}, \cdots, C_{m}$ of $|C|$ which correspond to the ideals $\mathfrak{A}_{0}, \mathfrak{A}_{1}, \ldots, \mathfrak{A}_{m}$.

Let $F$ be the algebraic subvariety of $V$ defined by the ideal $\mathfrak{F}=\left(\mathfrak{A}_{0}, \mathfrak{A}_{1}, \cdots, \mathfrak{A}_{m}\right)$, or rather, by the radical of this ideal. The variety $F$ is of dimension at most $r-2$ and is common to $C_{0}, C_{1}, \cdots, C_{m}$. We show that $F$ is the base manifold of the linear system $|C|$, that is, that $F$ lies on each $C_{(\lambda)}$. (This is not obvious, because of the presence of the factor $\mathfrak{M}$.)

Let $\mathfrak{p}$ be a minimal ideal of $\mathfrak{F}$ and let us show that the assumption $\mathfrak{A}_{(\lambda)} \not \equiv 0(\mathfrak{p})$ leads to a contradiction. Let $\beta$ be an element of $\mathfrak{A}_{(\lambda)}$ not in $\mathfrak{p}$. Since $\phi_{i} \equiv 0(\mathfrak{M})$, it follows $\left({ }^{30}\right)$, by $(9)$, that $\beta \phi_{i} \equiv 0\left(\phi_{(\lambda)}\right), i=0,1, \cdots, m$. Let $\beta \phi_{i}=\beta_{i} \phi_{(\lambda)}, \beta_{i} \in \mathrm{K}[\eta]$. This relation can be written as follows: $\beta \mathfrak{A}_{i}=\beta_{i} \mathfrak{A}(\lambda)$. By hypothesis $\mathfrak{A}_{i} \equiv 0(\mathfrak{p})$, but $\mathfrak{A}_{(\lambda)} \not \equiv 0(\mathfrak{p})$. Hence $\beta_{i} \equiv 0(\mathfrak{p})$, and therefore the relations $\beta \phi_{i}=\beta_{i} \phi_{(\lambda)}$ yield relations of the form

$$
\beta \phi_{i}=\beta_{i 0} \phi_{0}+\beta_{i 1} \phi_{1}+\cdots+\beta_{i m} \phi_{m}, \quad i=0,1, \cdots, m,
$$

where $\beta_{i j} \equiv 0(\mathfrak{p})$. From this we conclude that the determinant $\Delta=\left|\beta_{i j}-\delta_{i j} \beta\right|$ vanishes $\left(\delta_{i j}=0\right.$ if $\left.i \neq j, \delta_{i i}=1\right)$, and this is impossible since $\Delta \equiv \pm \beta^{m}(\mathfrak{p})$, whence $\Delta \not \equiv 0(p)$.

THEOREM 15. The base manifold $F$ of the linear system $|C|$ is also the fundamental locus of the birational transformation $T$, that is, $F$ has the property that any irreducible subvariety $W$ of $V$ which is fundamental for $T$ lies on $F$, and conversely.

Proof. Let $\mathfrak{p}$ be the homogeneous prime ideal in the ring $\mathrm{K}[\eta]$ which corresponds to $W$.

Assume $W \nsubseteq F$. Then at least one of the $m+1$ varieties $C_{i}$ does not contain $F$. Let, say, $F \nsubseteq C_{0}$, whence $\mathfrak{A}_{0} \neq \equiv 0(\mathfrak{p})$. We introduce the nonhomogeneous coordinates $\xi_{i}^{\prime}=\eta_{i}^{\prime} / \eta_{0}^{\prime}$ of the general point of $V^{\prime}$ and we denote, as usual,

$\left.{ }^{(30}\right)$ Strictly speaking, the congruences $\beta \equiv 0\left(\mathfrak{A}_{(\lambda)}\right), \phi_{i} \equiv 0(\mathfrak{M})$ do not necessarily imply that $\beta \phi_{i}$ is a multiple of $\phi(\lambda)$, since the equation (9) is only true to within an irrelevant component. However, in view of the regularity and the $(1,1)$ character of the birational correspondence between a locally normal variety and its derived normal variety, it is permissible to give the proof under the assumption that $V$ and $V^{\prime}$ are not only locally normal but normal. If $V$ is normal, then the equation (9) is exact. The same remark applies to the other theorems proved in this section. 
the ring $\mathrm{K}\left[\xi_{1}^{\prime}, \xi_{2}^{\prime}, \cdots, \xi_{m}^{\prime}\right]$ by $\mathfrak{o}^{\prime}$. We have $\left(\xi_{i}^{\prime}\right)=\mathfrak{A}_{i} / \mathfrak{A}_{0}$, whence $\xi_{i}^{\prime} \in Q(W)$. Thus the entire ring $D^{\prime}$ is contained in the quotient ring $Q(W)$, and this implies that $W$ is not fundamental.

Conversely, assume that $W$ is not fundamental. There corresponds then to $W$ a unique variety $W^{\prime}$ on $V^{\prime}$ and we have $Q\left(W^{\prime}\right) \subseteq Q(W)$. We may assume that $\eta_{0}^{\prime} \neq 0$ on $W^{\prime}$, and then we will have $\mathfrak{o}^{\prime} \subset Q\left(W^{\prime}\right)$, whence a fortiori $\mathrm{o}^{\prime} \subset Q(W)$. The fact that the quotients $\phi_{i} / \phi_{0}$ all belong to $Q(W)$ leads immediately to the conclusion that $\mathfrak{A}_{0} \not \equiv 0(\mathfrak{p})$. Hence $W$ does not lie on $F$, q.e.d.

While Theorem 15 gives full information about the location of the fundamental elements of a birational correspondence, the following theorem tells us where the irregular varieties are located:

THEOREM 16. If an irreducible subvariety $W^{\prime}$ of $V^{\prime}$ is irregular for $T^{-1}$ then $W^{\prime}$ lies on the total transform $T\{F\}$ of the fundamental locus $F$ of $T$. Conversely, if $W^{\prime}$ lies on $T\{F\}$, then it is either irregular or fundamental for $T^{-1}$.

The proof is immediate. For if $W^{\prime}$ is irregular, and if $W=T^{-1}\left(W^{\prime}\right)$, then $W$ is fundamental (Theorem 8 (C), II.3), whence $W \subseteq F$. Consequently, $W^{\prime}=T(W) \subseteq T\{F\}$. Conversely, if $W^{\prime} \subseteq T\{F\}$, then $W^{\prime}$ must correspond to some irreducible subvariety $W$ of $F$. Since $W$ is fundamental, $W^{\prime}$ cannot be regular, q.e.d.

The linear system $|C|$ which is defined by the linear family of forms $\phi_{(\lambda)}$ has always played an important part in the study of the birational correspondence $T$ with which this system is associated. Theorem 15 is one illustration of the geometric connection between $|C|$ and $T$. Another property of the linear system $|C|$ which follows in a straightforward fashion from the definition is the following: the birational correspondence $T$ transforms the linear system $|C|$ into the system of hyperplane sections of $V^{\prime}$. This statement should be intended in the following sense: a general member $C_{(\lambda)}$ of $|C|$ is regular and $T\left(C_{(\lambda)}\right)$ is the section $\Gamma_{(\lambda)}^{\prime}$ of $V^{\prime}$ with the hyperplane $\lambda_{0} y_{0}^{\prime}+\lambda_{1} y_{1}^{\prime}+\cdots+\lambda_{m} y_{m}^{\prime}$ $=0$. However, for special values of the $\lambda$ 's, it may very well happen that $C_{(\lambda)}$, or $\Gamma_{(\lambda)}^{\prime}$, or both, contain irreducible components which are irregular and which therefore correspond to fundamental varieties.

10. Isolated fundamental varieties. We assume as before that $V$ is locally normal and we keep the notation $V^{*}$ for the join of $V$ and $V^{\prime}$, and $T^{*}$ for the birational correspondence between $V$ and $V^{*}$.

Definition 8. Let $W_{1}^{*}, W_{2}^{*}, \cdots, W_{h}^{*}$ be the irreducible components of the total transform $T^{*}\{F\}$, where $F$ is the fundamental locus of $T^{*}$ (and hence also of $T)$ on $V$. The irreducible subvarieties $F_{i}=T^{*-1}\left(W_{i}^{*}\right)$ of $V$ are called the isolated fundamental varieties of $T$ (and also of $T^{*}$ ).

It is clear that the isolated fundamental varieties $F_{i}$ lie on the fundamental locus $F$. It is also not difficult to see that the irreducible components of $F$ are among the isolated fundamental varieties. For let $W$ be an irreducible compo- 
nent of $F$ and let $W^{*}$ be an irreducible component of the transform $T^{*}[W]$. We have $W^{*} \subseteq T^{*}\{F\}$, since $T^{*-1}\left(W^{*}\right)=W \subseteq F$. Consequently $W^{*}$ lies on one of the varieties $W_{i}^{*}$, say $W^{*} \subseteq W_{1}^{*}$. We have then $T^{*-1}\left(W^{*}\right) \subseteq T^{*-1}\left(W_{1}^{*}\right)=F_{1}$, that is, $W \subseteq F_{1}$. Since $W$ is a component of $F$, we conclude that $F_{1}=W$.

It is important to point out that in addition to the irreducible components of the fundamental locus $F$ there may exist other "embedded" isolated fundamental varieties, which are proper subvarieties of the irreducible components of $F$. Thus in the three-dimensional case we may have a fundamental curve $\Gamma$ on $V$ to which there corresponds a surface on $V^{*}$, and on that fundamental curve $\Gamma$ there may exist some special point $P$ to which there also corresponds a surface on $V^{*}$. This point $P$ must be regarded as an isolated fundamental point, although it is embedded in the fundamental curve $\Gamma$. The term "isolated" refers not to the position of the point $P$ with respect to the fundamental locus $F$ but to its role in the birational correspondence $T$.

By the main theorem each irreducible component $W_{i}^{*}$ of $T^{*}\{F\}$ is of higher dimension than the corresponding isolated fundamental variety $F_{i}$. Under certain conditions it is possible to assert that each $W_{i}^{*}$ is of dimension $r-1$. We proceed to find such conditions.

Let $\eta_{i j}$ denote as usual the homogeneous coordinates of the general point of the join $V^{*}$, where $\eta_{i j}=\eta_{i} \phi_{j}$ (see II.5 and II.9). Let us consider quite generally an arbitrary homogeneous ideal $\left(g_{0}, g_{1}, \cdots, g_{h}\right)$ in the ring $\mathrm{K}\left[\eta_{0}, \eta_{1}, \cdots, \eta_{n}\right]$, where each $g_{i}$ is a form, say of degree $\nu_{i}$. We put

$$
g_{i j}=g_{i}\left(\eta_{0 j}, \eta_{1 j}, \cdots, \eta_{n j}\right), \quad i=0,1, \cdots, h ; j=0,1, \cdots, m .
$$

The $(m+1)(h+1)$ forms $g_{i j}$ in the $\eta_{i j}$ 's generate a homogeneous ideal in the ring $\mathrm{K}\left[\eta_{00}, \eta_{10}, \cdots, \eta_{n m}\right]$.

Lemma 7. If $N$ is the subvariety of $V$ defined by the ideal $\left(g_{0}, g_{1}, \cdots, g_{h}\right)$ and if $N^{*}$ is the subvariety of $V^{*}$ defined by the ideal $\left(g_{00}, \cdots, g_{h m}\right)$, then $N^{*}$ is the total transform of $N$, that is, $N^{*}=T^{*}\{N\}$.

Proof. Let $W$ and $W^{*}$ be two corresponding irreducible subvarieties of $V$ and $V^{*}$, respectively. We have to show that $W^{*} \subseteq N^{*}$, if and only if $W \subseteq N$.

Assume that $W \subseteq N$. Without loss of generality we may assume that $\eta_{0} \neq 0$ on $W$. Then if $v$ denotes a valuation of centers $W$ and $W^{*}$, we will have:

$$
v\left(\eta_{i} / \eta_{0}\right) \geqq 0, \quad v\left(g_{i} / \eta_{0}^{\nu_{i}}\right)>0 .
$$

Also without loss of generality we may assume that $v\left(\phi_{i} / \phi_{0}\right) \geqq 0$, for $i=1,2, \cdots, m$. We will have then $v\left(\eta_{i j} / \eta_{00}\right)=v\left(\eta_{i} / \eta_{0}\right)+v\left(\phi_{j} / \phi_{0}\right) \geqq 0$. By $(10)$, we can write:

$$
g_{i j}=g_{i} \phi_{j}^{\prime \prime},
$$

and hence $g_{i j} / \eta_{00}^{\nu_{i}}=g_{i} / \eta_{0}^{\nu_{i}} \cdot\left(\phi_{j} / \phi_{0}\right)^{\nu_{i}}$. Consequently $v\left(g_{i j} / \eta_{00}^{\nu_{i}}\right)>0$, by (11), and this shows that $W^{*} \subseteq N^{*}$. 
Conversely, assume that $W^{*} \subseteq N^{*}$. Then if $\eta_{00} \neq 0$ on $W^{*}$, we find that $\eta_{0} \neq 0$ on $W$ (since $\eta_{i} / \eta_{0}=\eta_{i 0} / \eta_{00}$ ). On the other hand we have: $g_{i 0} / \eta_{00}^{\nu_{i}}=g_{i} / \eta_{0}^{\nu_{i}}$, whence $v\left(g_{i} / \eta_{0}^{\nu_{i}}\right)>0$, that is, $W \subseteq N$, q.e.d.

We now apply the lemma to the case in which $N$ is given by the ideal $\left(\phi_{0}, \phi_{1}, \cdots, \phi_{m}\right)$. Then $N^{*}$ is given by the ideal $\left(\phi_{00}, \phi_{10}, \cdots, \phi_{m m}\right)$, where

$$
\phi_{i j}=\phi_{i}\left(\eta_{0 j}, \eta_{1 j}, \cdots, \eta_{n j}\right) \text {. }
$$

The relations (12) now yield: $\phi_{i j}=\phi_{i} \phi_{j}^{\nu}$, where $\nu$ is the common degree of the form $\phi_{i}$. From these relations we deduce the following: $\phi_{i j}^{\nu+1}=\phi_{i t} \phi_{j j}^{\nu}$, and consequently the two ideals $\left(\phi_{00}, \phi_{10}, \cdots, \phi_{m m}\right)$ and $\left(\phi_{00}, \phi_{11}, \cdots, \phi_{m m}\right)$ have the same radical. Therefore $N^{*}$ is also defined by the ideal $\left(\phi_{00}, \phi_{11}, \cdots, \phi_{m m}\right)$. Now we have $\phi_{i i} / \phi_{j j}=\left(\phi_{i} / \phi_{j}\right)^{\nu+1}=\eta_{k i}^{\nu+1} / \eta_{k j}^{\nu+1}$, for any $i, j=0,1, \cdots, m$; $k=0,1, \cdots, n$. Therefore each irreducible component of $N^{*}$ at which $\eta_{k j} \neq 0$, for some $k$ and $j$, is also a component of the principal ideal $\left(\phi_{i j}\right)$ and is therefore $(r-1)$-dimensional. Consequently $N^{*}$ is pure $(r-1)$-dimensional.

In view of the formulas (9) of II.9, the variety of the ideal $\left(\phi_{0}, \phi_{1}, \cdots, \phi_{m}\right)$ consists of the $(r-1)$-dimensional variety of the ideal $\mathfrak{M}$ and of the fundamental locus $F$. We therefore can assert that $T^{*}\{F\}$ is pure $(r-1)$-dimensional if $\mathfrak{M}$ is the unit ideal. The hypothesis $\mathfrak{M}=(1)$ implies that each member $C_{(\lambda)}$ of the linear system $|C|$ associated with the birational correspondence is complete intersection of $V$ with a hypersurface of the ambient projective space, namely with the hypersurface $\lambda_{0} \phi_{0}\left(y_{0}, y_{1}, \cdots, y_{n}\right)+\lambda_{1} \phi_{1}\left(y_{0}, y_{1}, \cdots, y_{n}\right)$ $+\cdots+\lambda_{m} \phi_{m}\left(y_{0}, y_{1}, \cdots, y_{n}\right)=0$.

Conversely, let us assume that each $C_{(\lambda)}$ is complete intersection. Then in particular $C_{0}$ is complete intersection, whence the ideal $\mathfrak{A}_{0}$ is a principal ideal, say $\mathfrak{A}_{0}=\left(\psi_{0}\right)$. We have: $\phi_{i} \psi_{0} / \phi_{0}=\mathfrak{M A}_{i} \cdot \mathfrak{A}_{0} / \mathfrak{M A}_{0}=\mathfrak{A}_{i}$, that is, $\phi_{i} \psi_{0} / \phi_{0}$ is an integral ideal. Consequently the quotients $\phi_{i} \psi_{0} / \phi_{0}$ are forms in the $\eta$ 's, say $\phi_{i} \psi_{0} / \phi_{0}=\psi_{i}$. The forms $\psi_{0}, \psi_{1}, \cdots, \psi_{m}$ are proportional to the forms $\phi_{0}, \phi_{1}, \cdots, \phi_{m}$, and the linear system $|C|$ is also defined by the linear family of forms $\lambda_{0} \psi_{0}+\lambda_{1} \psi_{1}+\cdots+\lambda_{m} \psi_{m}$. If we use the $\psi$ 's instead of the $\phi$ 's, we will have $\mathfrak{M}=(1)$, and we reach again the conclusion that $T^{*}\{F\}$ is pure $(r-1)$-dimensional.

We can go a step further. Let us point out that if we define a projective model $V_{\rho}^{\prime}$ by the condition that the homogeneous coordinates of its general point be given by a linear base of the forms of degree $\rho$ in $\eta_{0}^{\prime}, \eta_{1}^{\prime}, \cdots, \eta_{m}^{\prime}$ then $V^{\prime}$ and $V_{\rho}^{\prime}$ are in regular birational correspondence (Lemma 5, II.2). The transition from $V^{\prime}$ to $V_{\rho}^{\prime}$ is equivalent to passing from the linear system $|C|$ to the least linear system which contains as members all sets of $\rho C$ 's. Hence, by the preceding result, we conclude that if a sufficiently high multiple of a $C$ is complete intersection, then $T^{*}\{F\}$ is pure $(r-1)$-dimensional.

In order to conclude with a similar result of a local character, let $F_{1}$ be any isolated fundamental variety of $T$, and let us assume that $C$ is locally, at $F_{1}$, complete intersection. By that we mean that some hypersurface cuts $V$ 
along $C$ and along a residual variety which does not contain $F_{1}$. If we replace the $\phi$ 's by a suitable set of proportional forms, we may arrange matters so that the variety of the ideal $\mathfrak{M}$ does not contain $F_{1}$. Since $N=M+F$, it is clear that $T^{*}\{N\}=T^{*}[M]+T^{*}\{F\}$, where we write $T^{*}[M]$ instead of $T^{*}\{M\}$, since $T^{*}\{M\}-T^{*}[M]$ lies on $T^{*}\{F\}$ (Theorem 13, II.6). Since $F_{1} \Phi M$, no component of $T^{*}\left[F_{1}\right]$ can lie on $T^{*}[M]$. It follows that the irreducible components of $T^{*}\{F\}$ which correspond to the isolated fundamental variety $F_{1}$ are also components of $T^{*}\{N\}$, and hence are $(r-1)$-dimensional. The same conclusion is reached if we assume that some sufficiently high multiple of $C$ is complete intersection locally at $F_{1}$.

The above results refer to $V$ and to the join of $V$ and $V^{\prime}$. In particular, if the birational correspondence $T$ has no fundamental elements on $V^{\prime}$ then $V^{\prime}$ may play the role of $V^{*}$, since $V^{\prime}$ and $V^{*}$ are then in regular birational correspondence. We reassume our results in the following theorem:

THEOREM 17. If a birational correspondence $T$ between two locally normal $r$-dimensional varieties $V$ and $V^{\prime}$ has no fundamental elements on $V^{\prime}$ and if $F$ denotes the fundamental locus of $T$ on $V$, then an irreducible component of $T\{F\}$ is of dimension $r-1$, provided the corresponding isolated fundamental variety $F_{1}$ has the property that the members of the linear system $|C|$ associated with $T$, or their sufficiently high multiples, are complete intersections locally, at $F_{1}$.

Corollary. To an isolated simple fundamental variety there always corresponds an $(r-1)$-dimensional variety on $V^{\prime}$ (see van der Waerden [6, p. 154]).

For locally, at a simple subvariety of $V$, every $(r-1)$-dimensional subvariety of $V$ is complete intersection $\left({ }^{31}\right)$.

11. Monoidal transformations. Given a homogeneous ideal $\mathfrak{A}$ in the ring $\mathrm{K}\left[\eta_{0}, \eta_{1}, \cdots, \eta_{n}\right]$ of homogeneous coordinates of the general point of $V$, it is possible to associate with $\mathfrak{A}$ an infinite set $S$ of birational transforms of $V$ such that: (1) the birational correspondence between $V$ and any variety $V^{\prime}$ of the set has no fundamental elements on $V^{\prime}$ and such that (2) any two varieties of the set are in regular birational correspondence. The varieties $V^{\prime}$ of the set $S$ shall be defined as follows. Let us take a base of $\mathfrak{A}$ consisting of forms of least possible degrees, and let $a$ be the highest degree of the forms in that base. We define $V^{\prime}$ by its general point $\left(\phi_{0}, \phi_{1}, \cdots, \phi_{m}\right)$, where the $\phi$ 's form a linear base for the forms of a given degree $\nu$ in $\mathfrak{A}$ and where we impose on $\nu$ the condition: $\nu \geqq a+1$. For $\nu=a+1, a+2, \cdots$, we get an infinite set of models $V^{\prime}$, and this is our set $S$. We shall denote these models by $V_{a+1}^{\prime}, V_{a+2}^{\prime}, \cdots$ :

(31) For the case of algebraically closed ground fields of characteristic zero see our paper $[8$, p. 664$]$. There the proof is given explicitly for surfaces only, but actually exactly the same proof applies to higher varieties. For ground fields which are not algebraically closed or which are of characteristic $p$, the statement can be derived from the following result obtained by Irvin Cohen in his dissertation: if the characteristic of a complete $p$-series ring coincides with the characteristic of its residue field, the ring is a power series ring over a field. 
First of all it is clear that each $V_{\nu}^{\prime}$ is birationally equivalent to $V$. For $\mathfrak{A}$ contains at least one form $\psi$ of degree $\nu-1$ so that the products $\eta_{0} \psi, \eta_{1} \psi, \cdots, \eta_{n} \psi$ can be identified with $n+1$ of the $\phi$ 's. This shows that the quotients $\phi_{i} / \phi_{0}$ generate the field $\Sigma$.

Since $\mathfrak{A}$ has a basis consisting of forms of degree at most $a$, it follows that if $\left(\phi_{0}, \phi_{1}, \cdots, \phi_{m}\right)$ is a basis for the forms in $\mathfrak{A}$ of degree $\nu$, then the products $\eta_{i} \phi_{j}$ constitute a basis for the forms in $\mathfrak{A}$ which are of degree $\nu+1$. This holds true also for $\nu=a$. From this it follows that $V_{\nu+1}$ is the join of $V$ and $V_{\nu}$, provided $\nu \geqq a+1$. Therefore the birational correspondence between $V$ and $V$, has no fundamental points on $V_{\nu}$, provided $\nu \geqq a+2$. But then $V_{\nu+1}$, the join of $V$ and $V_{\nu}$, is a regular birational transform of $V_{\nu}$, always provided that $\nu \geqq a+2$. As for the case $\nu=a+1$, we can still regard $V_{a+1}$ as the join of $V$ and $V_{a}$, although in this case $V_{a}$ need not be birationally equivalent to $V$. At any rate, the proof that the birational correspondence between $V$ and $V_{a+1}$ has no fundamental elements on $V_{a+1}$ is exactly the same as that given for the join in II.5.

Thus we may say that a given homogeneous ideal $\mathfrak{A}$ in the ring $\mathrm{K}\left[\eta_{0}, \eta_{1}, \cdots, \eta_{n}\right]$ determines, to within a regular birational transformation, a birational transform $V^{\prime}$ of $V$ such that the birational correspondence between $V$ and $V^{\prime}$ has no fundamental elements on $V^{\prime}$.

Let $N$ be the subvariety of $V$ defined by the ideal $\mathscr{A}$. It is quite clear that if $\nu \geqq a$, then the ideal generated by our base $\left(\phi_{0}, \phi_{1}, \cdots, \phi_{m}\right)$ differs from $\mathfrak{A}$ only by an irrelevant component. Hence, by Theorem 15 of II.9, we conclude that if $N$ is of the dimension at most $r-2$, then $N$ is the fundamental locus $F$ of the birational correspondence between $V$ and $V^{\prime}$. In particular, if $N$ is empty, that is, if $\mathfrak{A}$ is an irrelevant ideal, then $V^{\prime}$ is a regular transform of $V$.

If, however, $N$ is of dimension $r-1$, then the fundamental locus $F$ will consist of the irreducible components of $N$ which are of dimension less than $r-1$ and possibly of some proper subvarieties of the $(r-1)$-dimensional components of $N$. Thus, even in the case in which $N$ is pure $(r-1)$-dimensional, it may very well happen that $F$ is not empty. According to Theorem 15, this will happen if the residual intersections of the hypersurfaces $\phi_{i}=0$ $(i=0,1, \cdots, m)$ with $V$, outside $N$, have a base manifold on $N$.

Let now $\mathfrak{A}_{1}$ be another homogeneous ideal in the ring $\mathrm{K}\left[\eta_{0}, \eta_{1}, \cdots, \eta_{n}\right]$. If $W$ is an irreducible subvariety of $V$ given by a prime ideal $\mathfrak{p}$, we shall say that $\mathfrak{A}$ and $\mathfrak{A}_{1}$ coincide locally at $W$, if the two ideals differ only by primary components whose associated prime ideals are not multiples of $\mathfrak{p}$. In other words, $\mathfrak{A}$ and $\mathfrak{A}_{1}$ coincide locally at $W$ if they give rise to one and the same ideal in the quotient ring of $W$ (see I.1).

Lemma 8. If $\mathfrak{A}$ and $\mathfrak{A}_{1}$ coincide locally at an irreducible subvariety $W$ of $V$ and if $V^{\prime}$ and $V_{1}^{\prime}$ are the birational transforms of $V$ which are determined (to within a regular birational transformation), respectively, by $\mathfrak{A}$ and by $\mathfrak{A}_{1}$, then 
any irreducible subvariety of $V^{\prime}$ which corresponds to $W$ is regular for the birational correspondence between $V^{\prime}$ and $V_{1}^{\prime}$.

Proof. Let $\mathfrak{A}^{*}$ be the ideal which is obtained from either $\mathfrak{A}$ or $\mathfrak{A}_{1}$ by the omission of all primary components whose associated prime ideals are not multiples of $\mathfrak{p}$. Let $V^{*}$ be the birational transform of $V$ determined by the ideal $\mathfrak{A}^{*}$. It is sufficient to prove the lemma for $\mathfrak{A}$ and $\mathfrak{A}^{*}$, and for $\mathfrak{A}_{1}$ and $\mathfrak{A}^{*}$. We shall prove it, for instance, for $\mathfrak{A}$ and $\mathfrak{U}^{*}$.

Let $W^{\prime}$ be an irreducible subvariety of $V^{\prime}$ which corresponds to $W$. We have to prove that $W^{\prime}$ is regular for the birational correspondence between $V^{\prime}$ and $V^{*}$. Let $\phi_{0}, \phi_{1}, \cdots, \phi_{m}$ be a linear base of the forms of degree $\nu$ which belong to $\mathfrak{A}$. Since $\mathfrak{A} \subseteq \mathfrak{U}^{*}$, we may complete this base to a linear base $\phi_{0}, \phi_{1}, \cdots, \phi_{m}, \phi_{m+1}, \cdots, \phi_{m+\mu}$ for the forms of degree $\nu$ which belong to the ideal $\mathfrak{A}^{*}$. We take $\nu$ sufficiently high, so that $\left(\phi_{0}, \phi_{1}, \cdots, \phi_{m}\right)$ and $\left(\phi_{0}, \phi_{1}, \cdots, \phi_{m}, \phi_{m+1}, \cdots, \phi_{m+\mu}\right)$ are the general points, respectively, of $V^{\prime}$ and $V^{*}$.

Let $v$ be any valuation whose center on $V^{\prime}$ is $W^{\prime}$ and whose center on $V$ is $W$. We may assume that $v\left(\phi_{i} / \phi_{0}\right) \geqq 0, i=1,2, \cdots, m$ whence $W^{\prime}$ is at finite distance with respect to the nonhomogeneous coordinates $\xi_{i}^{\prime}=\phi_{i} / \phi_{0}$, $i=1,2, \cdots, m$, of the general point of $V^{\prime}$. Let $W^{*}$ be the center of $v$ on $V^{*}$. By our definition of the ideal $\mathfrak{A}^{*}$, there exists a form $g\left(\eta_{0}, \eta_{1}, \cdots, \eta_{n}\right)$ such that $g \cdot \mathfrak{A}^{*} \equiv 0(\mathfrak{R})$ and such that $g \neq 0$ on $W$. We have then, in particular: $g \phi_{m+j}=A_{0} \phi_{0}+A_{1} \phi_{1}+\cdots+A_{m} \phi_{m}, j=1,2, \cdots, \mu$, where $A_{0}, A_{1}, \cdots, A_{m}$ are forms in $\eta_{0}, \eta_{1}, \cdots, \eta_{n}$, of the same degree as $g$. We write:

$$
\phi_{m+j} / \phi_{0}=\frac{A_{0}}{g}+\frac{A_{1}}{g} \cdot \frac{\phi_{1}}{\phi_{0}}+\cdots+\frac{A_{m}}{g} \cdot \frac{\phi_{m}}{\phi_{0}} .
$$

Since $g \neq 0$ on $W$ we have $v\left(A_{i} / g\right) \geqq 0, i=0,1, \cdots, m$. Since also $v\left(\phi_{i} / \phi_{0}\right) \geqq 0$, it follows from the above relation (13) that $v\left(\phi_{m+j} / \phi_{0}\right) \geqq 0$. Hence $W^{*}$ is at finite distance with respect to the nonhomogeneous coordinates $\xi_{1}^{\prime}, \xi_{2}^{\prime}, \cdots, \xi_{m_{+\mu}}^{\prime}$ of the general point of $V^{*}$, where $\xi_{i}^{\prime}=\phi_{i} / \phi_{0}$. Since the ring $\mathrm{K}\left[\xi_{1}^{\prime}, \xi_{2}^{\prime}, \cdots, \xi_{m}^{\prime}\right]$ is a subring of the ring $\mathrm{K}\left[\xi_{1}^{\prime}, \xi_{2}^{\prime}, \cdots, \xi_{m+\mu}^{\prime}\right]$, it follows that $Q\left(W^{\prime}\right) \subseteq Q\left(W^{*}\right)$.

On the other hand, since $g \neq 0$ on $W$, the quotients $A_{i} / g$ belong to the quotient ring $Q(W)$. Since $Q(W) \subseteq Q\left(W^{\prime}\right)$ and since also the quotients $\phi_{i} / \phi_{0}$, $i=1,2, \cdots, m$, are in $Q\left(W^{\prime}\right)$, we conclude, by (13), that the entire ring $\mathrm{K}\left[\xi_{1}^{\prime}, \xi_{2}^{\prime}, \cdots, \xi_{m_{+}}^{\prime}\right]$ is contained in $Q\left(W^{\prime}\right)$. From this it follows immediately that $Q\left(W^{*}\right) \subseteq Q\left(W^{\prime}\right)$, whence $Q\left(W^{*}\right)=Q\left(W^{\prime}\right)$, q.e.d.

CoROllary. If $\mathfrak{A}$ and $\mathfrak{A}_{1}$ differ only by an irrelevant primary component, then $V^{\prime}$ and $V_{1}^{\prime}$ are in regular birational correspondence.

From the above general consideration we pass to the special case which interests us, namely to the case in which the given homogeneous ideal $\mathfrak{A}$ is a 
prime ideal $\mathfrak{p}$, of dimension $s, 0 \leqq s \leqq r-2$. Let $W$ be the irreducible subvariety of $V$ defined by $\mathfrak{p}$. The birational transformation $T$ determined by the ideal $\mathfrak{p}$ (that is, by a linear base of forms of sufficiently high degree in $\mathfrak{p}$ ) is called a monoidal transformation of center $W$. In the special case when $W$ is a point $P$ the transformation is called quadratic (of center $P$ ). The birational transform $V^{\prime}$ of $V$, under a monoidal transformation of given center, is determined to within a regular birational correspondence. The center $W$ of a monoidal transformation is the fundamental locus of the transformation. Moreover, from Theorem 17, II.10, it follows that in the present case $T\{W\}$ is pure $(r-1)$-dimensional. However, it should be pointed out that $T\{W\}$ may very well be reducible and-this is significant-some components of $T\{W\}$ may correspond to proper subvarieties of $W$. In other words, the center $W$ of a monoidal transformation is not necessarily the only isolated fundamental variety of the transformation $\left({ }^{32}\right)$. We shall see presently that this complication arises only if $W$ carries some singular points of $V$ or if $W$ itself has singularities.

Of special importance in applications are monoidal transformations with simple center, that is, with center at a simple subvariety $W$ of $V$. The special case of a quadratic transformation with simple center has been considered in our paper [11]. The results established there carry over to monoidal transformations with simple center, in view of the following considerations. Let the ground field $\mathrm{K}$ be extended by the adjunction of $s$ elements of $Q(W)$ which are algebraically independent on $W$. With respect to this new ground field $K_{1}$, the variety $W$ becomes a (simple) point and the monoidal transformation $T$ becomes a quadratic transformation. Therefore certain properties of the monoidal transformation $T$, over $\mathbf{K}$, can be deduced from corresponding properties of the quadratic transformation over $K_{1}$. However, only such properties of $T$ can be deduced in this fashion as concern $W$ as a whole. What happens to special points or special subvarieties of $W$ requires new considerations. For instance, we have proved in the quoted paper [11] that if $T$ is a quadratic transformation with simple center $P$, then the transform $T[P]$ (which, since $P$ is a point, coincides with the total transform $T\{P\})$ is an irreducible, simple and $(r-1)$-dimensional subvariety of $V^{\prime}$ and, moreover, that every

$\left.{ }^{(32}\right)$ Here is an example. Let $V$ be the quadric hypersurface $u^{2}=y z$ in the 4-dimensional space of the variables $x, y, z, u$. This hypersurface has the double line $y=z=u=0$. Let $W$ be the line $x=y=u=0$. As nonhomogeneous coordinates of the monoidal transform $V^{\prime}$ of $V$ we can take the elements $x, y, z, u, x / u, y / u$. Let $\mathrm{o}^{\prime}$ be the ring of these coordinates and let $\mathrm{D}=\mathrm{K}[x, y, z, u]$. We have $\mathrm{D}^{\prime}=\mathrm{D}[x / u, y / u]=\mathrm{K}\left[x_{1}, y_{1}, z\right]$, where $x_{1}=x / u, y_{1}=y / u$. Here $\mathfrak{p}=(x, y, u)$ is the prime ideal of $W$ and we have $\mathfrak{o}^{\prime} \cdot \mathfrak{p}=\mathfrak{o}^{\prime} \cdot \boldsymbol{u}=\mathfrak{o}^{\prime} \cdot y_{1} z$, that is, that part of $T\{W\}$ which is at finite distance consists of two planes: $y_{1}=0$ and $z=0$ (note that the affine model $V^{\prime}$ is in regular birational correspondence with the affine space of the variables $\left.x_{1}, y_{1}, z\right)$. The first plane corresponds to $W$ (since $\mathfrak{o}^{\prime} \cdot y_{1} \cap \mathfrak{D}=\mathfrak{p}$ ). But the plane $z=0$ corresponds to the point $x=y$ $=z=u=0$. This point is imbedded in $W$, but according to our terminology must be regarded as an isolated fundamental point. 
point of $T[P]$ is likewise simple for $V^{\prime}$. Now when we pass from the ground field $\mathrm{K}$ to the ground field $\mathrm{K}_{1}$, we lose all those components of $T\{W\}$ which cannot be regarded as varieties over $K_{1}$, that is, all those components of $T\{W\}$ which correspond to proper subvarieties of $W$ (since on any proper subvariety of $W$ the $s$ elements which have been adjointed to $\mathrm{K}$ are algebraically dependent). Consequently, the correct extrapolation of the above result concerning quadratic transformations to monoidal transformations is the following:

THEOREM 18. If the center $W$ of a monoidal transformation $T$ is a simple subvariety of $V$, then the transform $\left.{ }^{33}\right) T[W]$ of $W$ is an irreducible, simple, $(r-1)$-dimensional subvariety of $V^{\prime}$, and every irreducible subvariety $W^{\prime}$ of $T[W]$ is also simple for $V^{\prime}$, provided $W^{\prime}=T(W)$.

The total transform $T\{W\}$ may possess components which are not components of $T[W]$ (even if $W$ is simple $\left({ }^{32}\right)$ ), and concerning those components we can assert nothing. Likewise $T[W]$ may contain points which are singular for $V$. Thus, if $V$ is three-dimensional and if $W$ is a curve, then $T[W]$ is a surface which may carry, in addition to a finite number of singular points of $V$, also a finite number of singular curves of $V$, but each such curve must correspond to a point of $W$.

The following theorem will show, among other things, that these complications can arise only from points or subvarieties of $W$ which are singular for $V$ or for $W$.

THEOREM 19. Let $W_{1}$ be an irreducible subvariety of $W$, of dimension $s_{1}$. If $W_{1}$ is simple both for $V$ and $W$, then $T\left[W_{1}\right]$ lies on $T[W]$, is irreducible, is of dimension $r-1-s+s_{1}$ and is simple both for $V^{\prime}$ and for $T[W]$. Moreover, every irreducible subvariety of $T\left[W_{1}\right]$ which corresponds to $W_{1}$ is likewise simple for $V^{\prime}, T[W]$ and also for $T\left[W_{1}\right]$.

Proof. By the usual device of ground field extension we can achieve a reduction to the case $s_{1}=0$. Therefore we assume that $W_{1}$ is a point $P$ of $W$, simple both for $V$ and $W$. It is then possible to select uniformizing parameters $t_{1}, t_{2}, \cdots, t_{r}$ at $P$ in such a fashion that $W$ be locally, at $P$, complete intersection of the $r-s$ hypersurfaces $\left({ }^{34}\right) t_{1}=0, t_{2}=0, \cdots, t_{r-s}=0$. Then

${ }^{(33)}$ Not the total transform $T\{W\}$ !

(34) Proof. Quite generally, the uniformizing parameters $t_{1}, t_{2}, \cdots, t_{r-p}$ of a simple $\rho$-dimensional subvariety $L$ of $V$ have the following property: if $g\left(t_{1}, t_{2}, \cdots, t_{r-p}\right)=0$ is a true homogeneous relation between these parameters, with coefficients in the quotient ring $Q(L)$, then all these coeffcients must be zero on $L$, that is, they are non-units of $Q(L)$. (See $[9$, p. 202, (15) and p. 207, (23)].) In view of this property and also because $Q(L)$ is a chain theorem ring in which the non-units form an ideal, the quotient ring of a simple subvariety is a $p$-series ring ( $p$-Reihenring) in the sense of Krull [3]. We shall therefore apply properties of $p$-series rings due to Krull.

Let $\Im$ denote the quotient ring of $P$ and let $m$ be the ideal of non-units in $\Im$. If $\tau_{1}, \tau_{2}, \cdots, \tau_{r}$ are uniformizing parameters of $P$, then we have $\mathfrak{m}=\Im \cdot\left(\tau_{1}, \tau_{2}, \cdots, \tau_{r}\right)$. If $\alpha$ is any element of $\Im$ and if $\alpha \equiv \mathfrak{o}\left(\mathfrak{m}^{h}\right), \alpha \neq D\left(\mathfrak{m}^{h+1}\right)$, then $\alpha$ can be written as a form $g_{h}\left(\tau_{1}, \tau_{2}, \cdots, \tau_{r}\right)$, of degree $h$, 
$t_{1}, t_{2}, \cdots, t_{r-s}$ will be uniformizing parameters for $W$ (that is, the ideal generated by $t_{1}, t_{2}, \cdots, t_{r-s}$ in $Q(W)$ will be the prime ideal of non-units), and the ideal generated by the same elements in $Q(P)$ will be the prime ideal of $W$ in $Q(P)$.

The uniformizing parameters $t_{1}, t_{2}, \cdots, t_{r-s}$ of $W$ are proportional to certain forms $\psi_{1}, \psi_{2}, \cdots, \psi_{r-s}$ in the homogeneous coordinates $\eta_{0}, \eta_{1}, \cdots, \eta_{n}$ of the general point of $V$. Since these uniformizing parameters belong to $Q(P)$, it follows that the factor of proportionality can be so selected that the ideal generated by the forms $\left(\psi_{1}, \psi_{2}, \cdots, \psi_{r-s}\right)$ coincide locally at $P$ with the prime ideal of $W$. Hence by Lemma 8 we can replace the transformation $T$ by the birational transformation defined by the ideal $\left({ }^{35}\right)\left(\psi_{1}, \psi_{2}, \cdots, \psi_{r-s}\right)$. Therefore we may assume that $T$, instead of being our original monoidal transformation of center $W$, is the birational transformation which carries $V$ into the variety $V^{\prime}$ whose general point is $\left(\eta_{01}, \eta_{11}, \cdots, \eta_{n, r-s}\right)$, where $\left({ }^{36}\right)$ $\eta_{i j}=\eta_{i} \psi_{j}$.

Without loss of generality we may assume that the point $P$ (and hence also $W$ ) is at finite distance with respect to the nonhomogeneous coordinates $\xi_{i}=\eta_{i} / \eta_{0}, i=1,2, \cdots, n$. Let $\Gamma^{\prime}$ denote an irreducible component of $T[P]$. For some value of $h, h=1,2, \cdots, r-s$, it will be true that $\Gamma^{\prime}$ is at finite distance with respect to the nonhomogeneous coordinates

$$
\xi_{i j}^{(h)}=\eta_{i} \psi_{j} / \eta_{0} \psi_{h}
$$

with coefficients in $\Im$. If the coefficients of this form are replaced by their residues mod $\mathfrak{m}$, one obtains a form in $\tau_{1}, \tau_{2}, \cdots, \tau_{r}$ with coefficients in the residue field $\Im / \mathfrak{m}$. The property of uniformizing parameters stated above implies that this form is uniquely determined by the element $\alpha$. This form is called by Krull the leading form of $\alpha$ [3, p. 207].

It is a straightforward matter to show that $r$ elements $t_{1}, t_{2}, \cdots, t_{r}$ are uniformizing parameters of $P$, that is, $\mathfrak{m}=\Im \cdot\left(t_{1}, t_{2}, \cdots, t_{r}\right)$, if and only if the leading forms of $t_{1}, t_{2}, \cdots, t_{r}$ are linear and linearly independent.

Let $\mathfrak{p}$ denote the prime ideal of $W$ in $\Im_{\text {and let }} \Im^{*}=\mathfrak{\Im} / \mathfrak{p}, \mathfrak{m}^{*}=\mathfrak{m} / \mathfrak{p}$. Then $\Im^{*}$ is the quotient ring of the point $P$, regarded as a point of $W$, and $\mathfrak{m}^{*}$ is the ideal of non-units of $\Im^{*}$. Since, by hypothesis, $P$ is a simple point of $W$, there exist $s$ elements in $\Im^{*}$, say $t_{r-s+1}^{*}, t_{r-s+2}^{*}, \cdots, t_{r}^{*}$ such that $\mathfrak{m}^{*}=\mathfrak{\Im}^{*} \cdot\left(t_{r-s+1}^{*}, t_{r-8+2}^{*}, \cdots, t_{r}^{*}\right)$. Let $t_{r-s+1}, t_{r-s+2}, \cdots, t_{r}$ be elements of $\Im$ whose $\mathfrak{p}$-residues are $t_{r-s+1}^{*}, t_{r-s+2}^{*}, \cdots, t_{r}^{*}$, respectively. We will have then: $\mathfrak{m}=\mathfrak{\Im} \cdot\left(\mathfrak{p}, t_{r-s+1}, t_{r-s+2}, \cdots, t_{r}\right)$. From this relation we draw the following consequences. In the first place it follows that the ideal $\mathfrak{p}$ must contain $r-s$ elements whose leading forms are linear and linearly independent. Let $t_{1}, t_{2}, \cdots, t_{r-\text { b }}$ be such $r-s$ elements of $\mathfrak{p}$. If $\Im^{\prime}$ is the perfect closure of $\Im$ (see Krull [3, p. 217]), then it is a straightforward matter to show that the ideal $\Im^{\prime} \cdot\left(t_{1}, t_{2}, \cdots, t_{r-s}\right)$ is prime. Therefore also the ideal $\Im \cdot\left(t_{1}, t_{2}, \cdots, t_{r-8}\right)$ is prime, since it is the contraction of the ideal $\Im^{\prime} \cdot\left(t_{1}, t_{2}, \cdots, t_{r-8}\right)$ (Krull $\left[3\right.$, Theorem 15]). Since the leading ideal of $\Im \cdot\left(t_{1}, t_{2}, \cdots, t_{r-s}\right)$ is of dimension $s$, it follows (Krull $\left[3\right.$, Theorem 8]) that also $\Im \cdot\left(t_{1}, t_{2}, \cdots, t_{r-8}\right)$ is of dimension $s$. Consequently this ideal coincides with $\mathfrak{p}$. We have therefore: $\mathfrak{m}=\Im \cdot\left(t_{1}, t_{2}, \cdots, t_{r-s}, t_{r-s+1}, \cdots\right.$, $\left.t_{r}\right), \Im \cdot \mathfrak{p}=\Im \cdot\left(t_{1}, t_{2}, \cdots, t_{r-s}\right)$, q.e.d.

${ }^{(35)}$ Note that if two ideals coincide locally at some $W$ they also coincide locally at any $W_{1}$ such that $W \subseteq W_{1}$.

( $\left.{ }^{36}\right)$ Since our new transformation behaves locally at $P$ as the given monoidal transformation, we could refer to our new transformation as being locally monoidal at $P$. 
of the general point of $V^{\prime}$. Let $\mathrm{o}_{h}^{\prime}$ denote the ring of these nonhomogeneous coordinates, and let $\mathfrak{D}$ denote, as usual, the ring $\mathrm{K}\left[\xi_{1}, \xi_{2}, \cdots, \xi_{n}\right]$. Since $\psi_{j} / \psi_{h}=t_{j} / t_{h}$, we find:

$$
\mathrm{o}_{h}^{\prime}=\mathrm{o}\left[t_{1} / t_{h}, t_{2} / t_{h}, \cdots, t_{r-8} / t_{h}\right] .
$$

Without loss of generality we may assume that $\Gamma^{\prime}$ is at finite distance with respect to the nonhomogeneous coordinates $\xi_{i j}^{(1)}$. For simplicity we shall drop the subscript 1 in the symbol $\mathfrak{o}_{1}^{\prime}$, that is, we shall use the symbol $\mathfrak{o}^{\prime}$ to denote the ring $\mathfrak{D}_{1}^{\prime}$.

Let $\mathfrak{p}_{0}$ denote the prime zero-dimensional $\mathfrak{D}$-ideal of the point $P$. We shall denote by $\mathfrak{p}$ the prime $\mathfrak{0}$-ideal of $W$. For clarity of exposition we divide our proof into several steps.

(1) We shall show first that the ideal $\mathfrak{D}^{\prime} \cdot \mathfrak{p}_{0}$ is prime. Let $\Im=\mathfrak{D}_{\mathfrak{p}_{0}}$ denote the quotient ring of $P$ and let $\Im^{\prime}=\Im^{\prime} \cdot \mathfrak{o}^{\prime}$. The ring $\Im^{\prime}$ is a quotient ring of $\mathfrak{o}^{\prime}$, namely $\Im^{\prime}=\mathfrak{o}_{S}^{\prime}$ where $S=\mathfrak{D}-p_{0}$. Therefore, in view of the relationship between the ideals in a ring and the ideals in its quotient ring, the ideal $\mathfrak{o}^{\prime} \cdot \mathfrak{p}_{0}$ is prime if and only if $\Im^{\prime} \cdot p_{0}$ is prime $\left.{ }^{(37}\right)$. We prefer to deal with the ring $\Im^{\prime}$ and to show that $\Im^{\prime} \cdot p_{0}$ is prime.

Since $\Im \cdot p_{0}=\Im \cdot\left(t_{1}, t_{2}, \cdots, t_{r}\right)$ and $t_{i}=t_{1} \cdot t_{i} / t_{1}, i=2,3, \cdots, r-s$, that is, $t_{2}, t_{3}, \cdots, t_{r-8}$ are multiplies of $t_{1}$ in $\mathfrak{D}^{\prime}$, it follows that

$$
\Im^{\prime} \cdot p_{0}=\Im^{\prime} \cdot\left(t_{1}, t_{r-8+1}, \cdots, t_{r}\right) .
$$

Any element $\alpha$ in $\Im^{\prime}$ can be written in the form:

$$
\alpha=\phi_{\rho}\left(t_{1}, t_{2}, \cdots, t_{r-\delta}\right) / t_{1}^{\rho},
$$

where $\phi_{\rho}$ is a form of degree $\rho$ in $t_{1}, t_{2}, \cdots, t_{r-s}$, with coefficients in $\Im$. Let $\beta$ be another element in $\Im^{\prime}$,

$$
\beta=\psi_{\sigma}\left(t_{1}, t_{2}, \cdots, t_{r-8}\right) / t_{1}^{\sigma},
$$

and let us assume that $\alpha \beta \equiv 0\left(\Im^{\prime} \cdot p_{0}\right)$. We will have a relation of the form:

$$
\begin{aligned}
\phi_{\rho}\left(t_{1}, t_{2}, \cdots,\right. & \left.t_{r-8}\right) \cdot \psi_{\sigma}\left(t_{1}, t_{2}, \cdots, t_{r-8}\right) \cdot t_{1}^{\mu} \\
& =\left[t_{1} f_{\mu}\left(t_{1}, t_{2}, \cdots, t_{r-8}\right)+t_{r-s+1} f_{\mu}^{(1)}\left(t_{1}, t_{2}, \cdots, t_{r-8}\right)+\cdots\right. \\
& \left.\quad+t_{r} f_{\mu}^{(8)}\left(t_{1}, t_{2}, \cdots, t_{r-8}\right)\right] t_{1}^{\rho+\sigma},
\end{aligned}
$$

where $f_{\mu}, f_{\mu}^{(1)}, \cdots, f_{\mu}^{(s)}$ are forms of degree $\mu$, with coefficients in $\Im$. The righthand side of this relation is a form of degree $\rho+\sigma+\mu+1$ in $t_{1}, t_{2}, \cdots, t_{r}$, with

$\left.{ }^{(37}\right)$ We have a $(1,1)$ isomorphic correspondence between the ideals in $\Im^{\prime}$ and those ideals in $\mathfrak{D}^{\prime}$ all prime ideals of which contract in $\boldsymbol{D}$ to $\mathfrak{p}_{0}$ or to multiples of $\mathfrak{p}_{0}$. Now since $\mathfrak{p}_{0}$ is a maximal ideal, every prime ideal of $\mathfrak{b}^{\prime} \cdot \mathfrak{p}_{0}$ contracts to $\mathfrak{p}_{0}$. Hence $\mathfrak{b}^{\prime} \cdot \mathfrak{p}_{0}$ and $\Im^{\prime} \cdot \mathfrak{p}_{0}$ are corresponding ideals in the above correspondence. 
coefficients in $\Im$. Hence by a well known property of uniformizing parameters $\left({ }^{34}\right)$ either all the coefficients of $\phi_{\rho}$ or all the coefficients of $\psi_{\sigma}$ 'must be elements of $\mathfrak{\Im} \cdot \mathfrak{p}_{0}$. Suppose that all the coefficients of $\phi_{\rho}$ are in $\mathfrak{Y} \cdot \mathfrak{p}_{0}$. Since $\Im \cdot p_{0}=\Im \cdot\left(t_{1}, t_{2}, \cdots, t_{r}\right)$, we will have for $\phi_{\rho}$ an expression of the form: $\phi_{\rho}=t_{1} \phi_{\rho}^{(1)}\left(t_{1}, t_{2}, \cdots, t_{r-8}\right)+t_{2} \phi_{\rho}^{(2)}\left(t_{1}, t_{2}, \cdots, t_{r-8}\right)+\cdots+t_{r} \phi_{\rho}^{(r)}\left(t_{1}, t_{2}, \cdots, t_{r-8}\right)$, where the $\phi_{\rho}^{j}$ are again forms of degree $\rho$, with coefficients in $\Im$. Since $t_{2}, \cdots, t_{r-s}$ are multiples of $t_{1}$ in $\Im^{\prime}$, we conclude immediately that $\phi_{\rho} / t_{1}^{\rho}$ is contained in the ideal $\Im^{\prime}\left(t_{1}, t_{r-s+1}, \cdots, t_{r}\right)$, that is, in view of (15), $\alpha \equiv 0\left(\Im^{\prime} \cdot p_{0}\right)$. This shows that $\Im^{\prime} \cdot p_{0}$ is a prime ideal, as was asserted( $\left.{ }^{38}\right)$.

(2) Let $\mathfrak{b}^{\prime} \mathfrak{p}_{0}=\mathfrak{p}^{\prime}$. We assert that the $\mathfrak{p}^{\prime}$-residues of $t_{2} / t_{1}, \cdots, t_{r-s} / t_{1}$ are algebraically independent (over $\mathrm{K}$ ). For a relation of algebraic dependence between these residues would imply a relation of the form:

$$
\phi_{\rho}\left(t_{1}, t_{2}, \cdots, t_{r-8}\right) / t_{1}^{\rho}=\sum_{i=1}^{r} t_{i} g_{\sigma}^{(i)}\left(t_{1}, t_{2}, \cdots, t_{r-8}\right) / t_{1}^{\sigma},
$$

where the $g_{\sigma}^{(t)}$ and $\phi_{\rho}$ are forms in $t_{1}, t_{2}, \cdots, t_{r-s}$, with coefficients in $\mathfrak{D}$, and where the coefficients of $\phi_{\rho}$ are not all in $\mathfrak{p}_{0}$. Such a relation, cleared of the denominators, is in contradiction with the property of uniformizing parameters stated above ${ }^{(34)}$.

From the fact that $\mathfrak{v}^{\prime} \cdot p_{0}$ is prime, follows that $\Gamma^{\prime}$ is the only irreducible component of $T[P]$ which is at finite distance with respect to the coordinates $\xi_{i j}^{(1)}$.

The fact that the $\mathfrak{p}^{\prime}$-residue of $t_{2} / t_{1}, \cdots, t_{r-s} / t_{1}$ are algebraically independent, in conjunction with the fact that $\mathfrak{p}^{\prime} \cap \mathfrak{o}$ is the zero-dimensional ideal $\mathfrak{p}_{0}$, implies that $\Gamma^{\prime}$ is of dimension $r-s-1$. Moreover, the algebraic independence of the quotients $t_{2} / t_{1}, t_{3} / t_{1}, \cdots, t_{r-s} / t_{1}$ implies in particular that they do not belong to $\mathfrak{p}^{\prime}$. Hence these quotients are units in the quotient ring $Q\left(\Gamma^{\prime}\right)$. But then also $t_{i} / t_{h} \in Q\left(\Gamma^{\prime}\right)$, for $i, h=1,2, \cdots, r-s$, whence the rings $\mathrm{D}_{h}^{\prime}$ (see (14)) belong to $Q\left(\Gamma^{\prime}\right)$. This shows that $\Gamma^{\prime}$ is at finite distance also with respect to the nonhomogeneous coordinates $\xi_{i j}^{(h)}$, for $h=1,2, \cdots, r-s$. Consequently, $\Gamma^{\prime}$ is the only irreducible component of $T[P]$, that is, $T[P]$ is irreducible: $T[P]=\Gamma^{\prime}$.

(3) Let $C^{\prime}$ denote the irreducible $(r-1)$-dimensional variety $T[W]$. We are interested in the quotient rings of $\Gamma^{\prime}$ and of $C^{\prime}$. On the basis of the preceding considerations we find immediately that every element of $Q\left(\Gamma^{\prime}\right)$ is of the form: $f_{\rho}\left(t_{1}, t_{2}, \cdots, t_{r-s}\right) / g_{\rho}\left(t_{1}, t_{2}, \cdots, t_{r-s}\right)$, where $f_{\rho}$ and $g_{\rho}$ are forms of like degree $\rho$, with coefficients in $\mathfrak{D}$, and where the coefficients of $g_{\rho}$ are not all in $\mathfrak{p}_{0}$. Similarly, making use of the remark in footnote 38 , or also directly from the properties of what we have called " $p$-adic divisor" in [11], we conclude that the elements of $Q\left(C^{\prime}\right)$ are all of the form $f_{\rho}\left(t_{1}, t_{2}, \cdots, t_{r-s}\right) / g_{\rho}\left(t_{1}, t_{2}, \cdots, t_{r-s}\right)$,

(38) Exactly the same proof could be applied toward proving the following: if $\mathfrak{p}$ is the prime

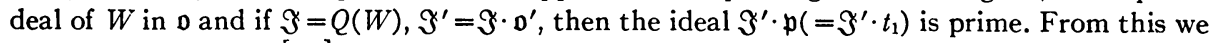
could conclude that $T[W]$ is irreducible (as asserted in Theorem 18) in exactly the same fashion as we concluded in the text that $T[P]$ is irreducible. 
with the same conditions on $f_{\rho}$ and $g_{\rho}$ as above, except that now the coefficients of $g_{\rho}$ must not all be in $\mathfrak{p}$, where $\mathfrak{p}$ is the prime ideal of $W$. Since $P \subseteq W$, it follows that $Q\left(\Gamma^{\prime}\right) \subseteq Q\left(C^{\prime}\right)$, that is, $T[P]$ lies on $T[W]$ (that is, $\Gamma^{\prime}$ lies on $C^{\prime}$ ).

Moreover, from the preceding considerations (see relation (15)), it follows that the prime ideal of non-units in $Q\left(\Gamma^{\prime}\right)$ has the basis $t_{1}, t_{r-s+1}, \cdots, t_{r}$, consisting of $s+1$ elements. Since $\Gamma^{\prime}$ is of dimension $r-s-1$, it follows that $\Gamma^{\prime}$ is a simple subvariety of $V^{\prime}$, and that $t_{1}, t_{r-s+1}, \cdots, t_{r}$ are uniformizing parameters of $\Gamma^{\prime}$. In a similar fashion we find that $t_{1}$ is a uniformizing parameter of the $(r-1)$-dimensional variety $C^{\prime}$, and since $t_{1}$ is among the uniformizing parameters of $\Gamma^{\prime}$, we conclude that $\Gamma^{\prime}$ is a simple subvariety of $C^{\prime}$.

(4) To complete the proof of our theorem we have only to show that every point $P^{\prime}$ of $\Gamma^{\prime}$ is simple for $V^{\prime}, C^{\prime}$ and $\Gamma^{\prime}$. To show that $P^{\prime}$ is simple for $V^{\prime}$ we have to exhibit $r$ uniformizing parameters at $P^{\prime}$. Let $\Delta$ be the residue class field of the point $P$, that is, let $\Delta=\mathfrak{o} / \mathfrak{p}$. Similarly let $\Delta^{\prime}$ be the residue class field of $P^{\prime}$. Here $\Delta$ and $\Delta^{\prime}$ are finite algebraic extensions of $\mathrm{K}$, and $\Delta^{\prime} \supseteq \Delta$ since $Q\left(P^{\prime}\right) \supseteq Q(P)$. Without loss of generality we may assume that $P^{\prime}$ is at finite distance with respect to the ring $\mathfrak{D}^{\prime}$ of nonhomogeneous coordinates $\left(\mathfrak{o}^{\prime}=\mathfrak{o}_{1}^{\prime}\right.$, see (14)), and is therefore given in $\mathfrak{D}^{\prime}$ by a prime zero-dimensional ideal $\mathfrak{p}_{0}^{\prime}$.

Let $c_{1}, c_{2}, \cdots, c_{n}$ be the $P$-residues of $\xi_{1}, \xi_{2}, \cdots, \xi_{n}$ respectively $\left(c_{i} \in \Delta\right)$ and let $d_{2}, d_{3}, \cdots, d_{r-s}$ be the $P^{\prime}$-residues of $t_{2} / t_{1}, t_{3} / t_{1}, \cdots, t_{r-s} / t_{1}\left(d_{i} \in \Delta^{\prime}\right)$. The element $d_{i}$ will be the root of an irreducible polynomial $f_{i}(z)$ with coefficients in $\Delta$. We replace the coefficients of $f_{i}(z)$ by arbitrary but fixed elements of $\mathfrak{D}$ of which they are residues. Let $F_{i}(z)$ be the polynomial with coefficients in $\mathrm{D}$ thus obtained, $i=2,3, \cdots \therefore, r-s$. If we assume that the polynomials $f_{1}(z)$ are all separable then we can conclude as in $[11$, p. 590] that the $r$ elements

$$
t_{1}, F_{2}\left(t_{2} / t_{1}\right), \cdots, F_{r-s}\left(t_{r-s} / t_{1}\right), t_{r-s+1}, \cdots, t_{r}
$$

are uniformizing parameters at $P^{\prime}$. Since these elements include the uniformizing parameters $t_{1}, t_{r-s+1}, \cdots, t_{r}$ of $\Gamma^{\prime}$ and the uniformizing parameter $t_{1}$ of $C^{\prime}$, the proof is complete.

However, if some or all of the polynomials $f_{i}(z)$ are inseparable, then the elements (16) are no longer uniformizing parameters at $P^{\prime}$ (compare with footnote 41). We shall therefore give here a new proof which applies both to the separable and non-separable case. We consider the residue class ring $\mathfrak{D}^{*}=\mathfrak{D}^{\prime} / \mathfrak{p}^{\prime}$, where $\mathfrak{p}^{\prime}$ is the prime $\mathfrak{D}^{\prime}$-ideal of $\Gamma^{\prime}$. Let $z_{2}, z_{3}, \cdots, z_{r-s}$ be the $\mathfrak{p}^{\prime}$-residues of $t_{2} / t_{1}, t_{3} / t_{1}, \cdots, t_{r-s} / t_{1}$. Since $\mathfrak{p}^{\prime} \cap_{\mathfrak{D}}=\mathfrak{p}_{0}$ and since we have shown earlier in this section that $z_{2}, z_{3}, \cdots, z_{r-s}$ are algebraically independent over $\mathrm{K}$, it follows from (14), for $h=1$, that $\mathrm{D}^{*}$ is a polynomial ring $\left({ }^{39}\right)$ over $\Delta$ :

(39) This shows incidentally that the field of rational functions on $\Gamma^{\prime}$ is a pure transcendental extension of $\Delta$, whence $\Gamma^{\prime}$ is a rational variety over $\Delta$. 


$$
\mathfrak{D}^{*}=\Delta\left[z_{2}, z_{3}, \cdots, z_{r-8}\right] .
$$

The rest of the proof will be based on the following lemma:

Lemma 9. In a polynomial ring $P_{n}=\Delta\left[x_{1}, x_{2}, \cdots, x_{n}\right]$ over an arbitrary field $\Delta$ every prime zero-dimensional ideal possesses a base consisting of $n$ elements $\left({ }^{40}\right)$.

Proof of the lemma. Since the lemma is trivially true for $n=1$, we proceed by induction with respect to $n$. Let $\mathfrak{p}$ be a prime zero-dimensional ideal in $P_{n}$ and let $f(z)$ be the irreducible polynomial in $\Delta[z]$ such that $f\left(x_{n}\right) \equiv 0(p)$. The residue class ring $P_{n-1}^{*}=P_{n} / f\left(x_{n}\right)$ is obviously a polynomial ring $\Delta^{*}\left[x_{1}, x_{2}, \cdots, x_{n-1}\right]$ over the field $\Delta^{*}=\Delta(\alpha)$, where $\alpha$ is a root of $f(z)$. The ideal $\mathfrak{p}^{*}=\mathfrak{p} / f\left(x_{n}\right)$ is prime and zero-dimensional in $P_{n-1}^{*}$. By our induction, there exist $n-1$ elements $\omega_{1}^{*}, \omega_{2}^{*}, \cdots, \omega_{n-1}^{*}$ in $P_{n-1}^{*}$ such that $p^{*}=P_{n-1}^{*}\left(\omega_{1}^{*}, \omega_{2}^{*}, \cdots, \omega_{n-1}^{*}\right)$. Let $\omega_{1}, \omega_{2}, \cdots, \omega_{n-1}$ be elements of $P_{n}$ whose residues modulo $f\left(x_{n}\right)$ are, respectively, $\omega_{1}^{*}, \omega_{2}^{*}, \cdots, \omega_{n-1}^{*}$. Then it is clear that $p=\left(\omega_{1}, \omega_{2}, \cdots, \omega_{n-1}, f\left(x_{n}\right)\right)$, q.e.d. $\left({ }^{41}\right)$.

We now apply our lemma. In the homomorphism $\mathfrak{o}^{\prime} \sim \mathfrak{o}^{*}$ the prime $\mathfrak{o}^{\prime}$-ideal $p_{0}^{\prime}$ of the point $P^{\prime}$ is mapped upon a prime zero-dimensional $0^{*}$-ideal $\mathfrak{p}_{0}^{*}$. By the lemma we have

$$
p_{0}^{*}=\left(\zeta_{2}^{*}, \zeta_{3}^{*}, \cdots, \zeta_{r-s}^{*}\right) .
$$

Let $\zeta_{2}^{\prime}, \zeta_{3}^{\prime}, \cdots, \zeta_{r-s}^{\prime}$ be elements of $\mathfrak{D}^{\prime}$ whose $\mathfrak{p}^{\prime}$-residues are respectively $\zeta_{2}^{*}, \zeta_{3}^{*}, \cdots, \zeta_{r-s .}^{*}$ Then we have

$$
\mathfrak{p}_{0}^{\prime}=\left(\mathfrak{p}^{\prime}, \zeta_{2}^{\prime}, \zeta_{3}^{\prime}, \cdots, \zeta_{r-8}^{\prime}\right) \text {. }
$$

Let $\Im_{1}^{\prime}$ be the quotient ring of $P^{\prime}$. Since $Q\left(P^{\prime}\right) \supset Q(P)=\Im$ and since $Q\left(P^{\prime}\right) \supset \mathfrak{v}^{\prime}$, the ring $\Im_{1}^{\prime}$ contains all the rings previously considered, that is, the rings $\mathfrak{D}, \mathfrak{p}^{\prime}, \Im$ and $\Im^{\prime}\left(\Im^{\prime}=\Im^{\prime} \cdot \mathfrak{p}^{\prime}\right)$. We have $\mathfrak{p}^{\prime}=\mathfrak{p}^{\prime} \mathfrak{p}_{0}$, whence $\Im_{1}^{\prime} \cdot \mathfrak{p}^{\prime}=\Im_{1}^{\prime} \cdot \mathfrak{p}_{0}$ $=\Im_{1}^{\prime} \cdot \Im \cdot p_{0}=\Im_{1}^{\prime} \cdot\left(t_{1}, t_{2}, \cdots, t_{r}\right)=\Im_{1}^{\prime} \cdot\left(t_{1}, t_{r-s+1}, \cdots, t_{r}\right)$ (since $t_{i} / t_{1} \in \mathfrak{o}^{\prime} \in \Im_{1}^{\prime}$, $i=2,3, \cdots, r-s)$. Substituting into (17) we find:

$$
\Im_{1}^{\prime} \cdot p_{0}^{\prime}=\Im_{1}^{\prime} \cdot\left(t_{1}, \zeta_{2}, \zeta_{3}, \cdots, \zeta_{r-8}, t_{r-s+1}, t_{r-8+2}, \cdots, t_{r}\right) .
$$

${ }^{\left({ }^{40}\right)}$ This lemma implies that every point $P$ of an affine (or of a projective) space over $\Delta$ is simple. The lemma gives, however, a stronger result, since it shows that uniformizing parameters at $P$ can be so selected that they generate the prime ideal of $P$ not only in $Q(P)$ but also in the polynomial ring. In other words: every point $P$ of an affine $n$-space is complete intersection of $n$ hypersurfaces. This result can be extended without any difficulties to projective spaces by a similar inductive argument.

(11) In the case of ground fields of characteristic zero we have used instead of the above lemma the following property of the polynomial ring $P_{n}$ : if $f_{i}(z)$ is the irreducible polynomial in $\Delta[z]$ such that $f_{i}\left(x_{i}\right) \equiv 0(\mathfrak{p})$, then the ideal $\left(f_{1}\left(x_{1}\right), f_{2}\left(x_{2}\right), \cdots, f_{n}\left(x_{n}\right)\right)$ is the intersection of prime zero-dimensional ideals, one of which is of course the ideal $\mathfrak{p}$ itself. In this case the $n$ polynomials $f_{i}\left(x_{i}\right)$ are uniformizing parameters for the point defined by $\mathfrak{p}$. This reasoning applies also in the case in which the polynomials $f_{i}\left(x_{i}\right)$ are separable. 
This exhibits $r$ uniformizing parameters at $P^{\prime}$, and since these parameters include the uniformizing parameters of $\Gamma^{\prime}$ and that of $C^{\prime}$, the proof is now complete.

Corollary. If all points of $W$ are simple both for $W$ and for $V$, then $T[W]=T\{W\}(T-a$ monoidal transformation of center $W)$ and $T[W]$ is irreducible, $(r-1)$-dimensional and all its points are simple both for $T[W]$ and $V^{\prime}$. Moreover, if $W$ is of dimension $s$, then $T[W]$ is covered by an s-dimensional algebraic system $\left\{\Gamma^{\prime}\right\}$ of $(r-s-1)$-dimensional varieties $\Gamma^{\prime}$ in $(1,1)$ correspondence with the points of $W$. Each $\Gamma^{\prime}$ is irreducible, rational and free from singularities, and through each point of $T[W]$ there passes a unique $\Gamma^{\prime}$.

\section{REFERENCES}

1. H. Grell, Beziehungen zwischen den Idealen verschiedener Ringe, Math. Ann. vol. 97 (1927).

2. W. Krull, Idealtheorie, Ergebnisse der Mathematik und ihrer Grenzgebiete, IV 3.

- 3. - Dimensionstheorie in Stellenringen, J. Reine Angew. Math., vol. 179 (1938).

4. S. MacLane and O. F. G. Schilling, Zero-dimensional branches of rank one on algebraic varieties, Ann. of Math. (2) vol. 40 (1939).

5. F. K. Schmidt, Über die Erhaltung der Kettensätze der Idealtheorie bei beliebigen endlichen Körpererweiterungen, Math. Zeit. vol. 41 (1936).

6. B. L. van der Waerden, Algebraische Korrespondenzen und rationale Abbildungen, Math. Ann. vol. 110 (1934).

7. O. Zariski, Some results in the arithmetic theory of algebraic varieties, Amer. J. Math. vol. 61 (1939). (1939).

8. - The reduction of the singularities of an algebraic surface, Ann. of Math. vol. 40 (1940).

9. - - Algebraic varieties over ground fields of characteristic zero, Amer. J. Math. vol. 62

10. Pencils on an algebraic variety and a new proof of a theorem of Bertini, Trans. Amer. Math. Soc. vol. 50 (1941).

11. - A simplified proof for the resolution of singularities of an algebraic surface, Ann. of Math. (2) vol. 43 (1942).

12. - Normal varieties and birational correspondences, Bull. Amer. Math. Soc. vol. 48

The Johns Hopkins University, BALTimore, MD. 